\title{
Axillary reverse mapping
}

Citation for published version (APA):

Beek, M. (2016). Axillary reverse mapping: towards selective axillary surgery in breast cancer patients. [Doctoral Thesis, Maastricht University]. Datawyse / Universitaire Pers Maastricht. https://doi.org/10.26481/dis.20161221mb

Document status and date:

Published: 01/01/2016

DOI:

10.26481/dis.20161221mb

Document Version:

Publisher's PDF, also known as Version of record

\section{Please check the document version of this publication:}

- A submitted manuscript is the version of the article upon submission and before peer-review. There can be important differences between the submitted version and the official published version of record.

People interested in the research are advised to contact the author for the final version of the publication, or visit the DOI to the publisher's website.

- The final author version and the galley proof are versions of the publication after peer review.

- The final published version features the final layout of the paper including the volume, issue and page numbers.

Link to publication

\footnotetext{
General rights rights.

- You may freely distribute the URL identifying the publication in the public portal. please follow below link for the End User Agreement:

www.umlib.nl/taverne-license

Take down policy

If you believe that this document breaches copyright please contact us at:

repository@maastrichtuniversity.nl

providing details and we will investigate your claim.
}

Copyright and moral rights for the publications made accessible in the public portal are retained by the authors and/or other copyright owners and it is a condition of accessing publications that users recognise and abide by the legal requirements associated with these

- Users may download and print one copy of any publication from the public portal for the purpose of private study or research.

- You may not further distribute the material or use it for any profit-making activity or commercial gain

If the publication is distributed under the terms of Article $25 \mathrm{fa}$ of the Dutch Copyright Act, indicated by the "Taverne" license above, 


\section{AXILLARY REVERSE MAPPING}

Towards selective axillary surgery in breast cancer patients
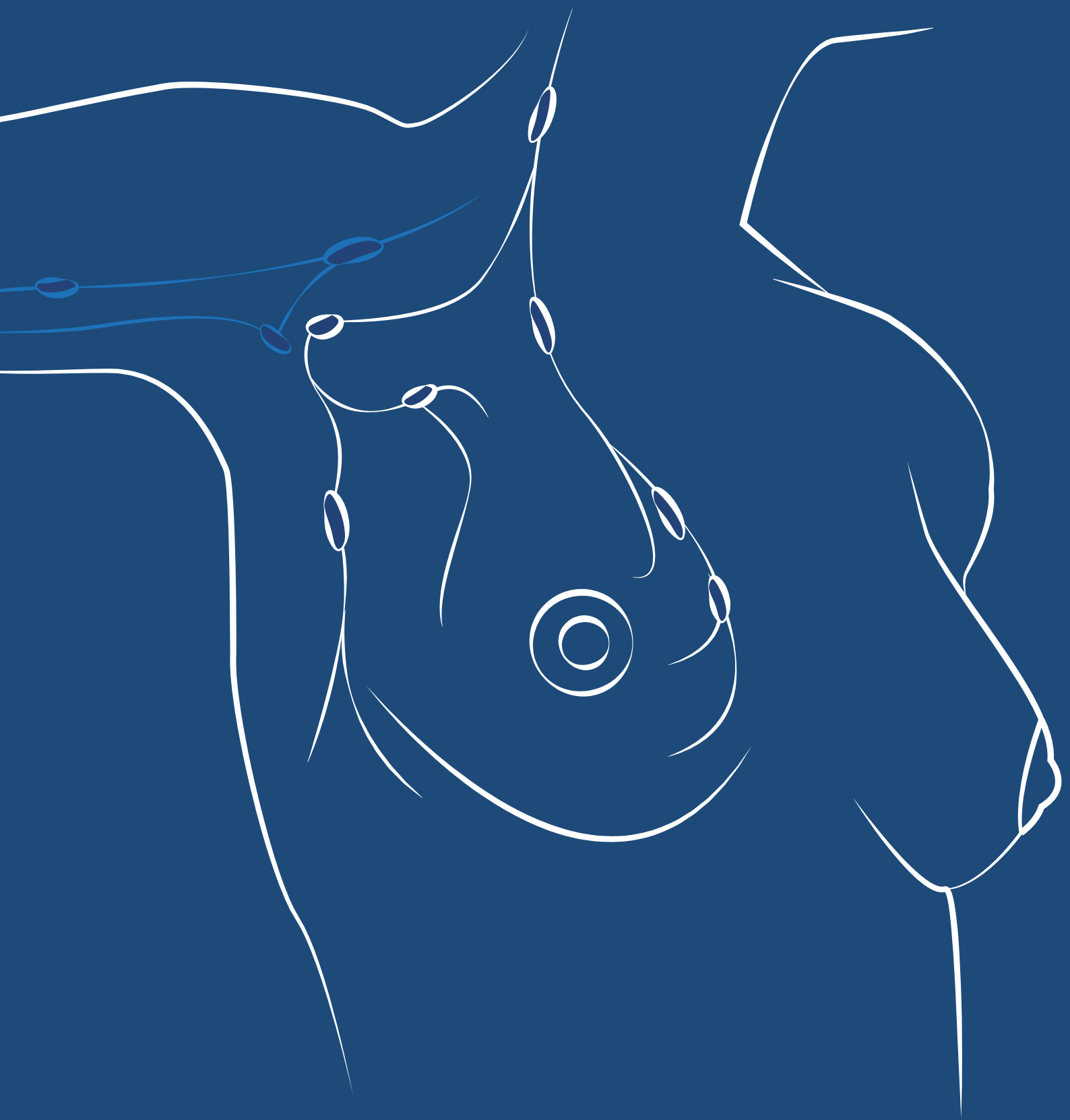

Martinus A. Beek 
Axillary reverse mapping: towards selective axillary surgery in breast cancer patients Martinus A. Beek

Thesis, University Maastricht, with a summary in Dutch

Proefschrift, Universiteit Maastricht, met een samenvatting in het Nederlands

(C) Maarten Beek, Maastricht 2016

No part of this book may be reproduced or transmitted in any form or by any means, without prior permission in writing by the author, or when appropriate, by the publishers of the publications.

Layout: Tiny Wouters

Cover design: Leon Neve

Production: Datawyse | Universitaire Pers Maastricht

ISBN: 9789461596161

Financial support for the publication of this thesis was kindly provided by: Raad van het bestuur Amphia ziekenhuis, Universiteit Maastricht, Pink Ribbon, Olympus Nederland B.V., ChipSoft, Novuqare and Sophens. 


\title{
Axillary reverse mapping \\ towards selective axillary surgery in \\ breast cancer patients
}

\author{
PROEFSCHRIFT \\ ter verkrijging van de graad van doctor aan de Universiteit Maastricht, \\ op gezag van de Rector Magnificus, Prof. dr. Rianne M. Letschert, \\ volgens het besluit van het College van Decanen, \\ in het openbaar te verdedigen \\ op woensdag 21 december 2016 om 16.00 uur \\ door \\ Martinus Anthonius Beek
}

geboren op 27 januari 1988 te Rotterdam 


\section{Promotor}

Prof. dr. H.J.T. Rutten

\section{Co-promotores}

Dr. E.J.T. Luiten, Amphia Ziekenhuis, Breda

Dr. A.C. Voogd

Dr. P.D. Gobardhan, Amphia Ziekenhuis, Breda

\section{Beoordelingscommissie}

Prof. dr. V.C.G. Tjan-Heijnen (voorzitter)

Prof. dr. C.H.C. Dejong

Dr. M.B.I. Lobbes

Prof. dr. Ph.M.P. Poortmans, Radboud Universitair Medish Centrum, Nijmegen

Prof. dr. E.J.Th. Rutgers, Antoni van Leeuwenhoek, Amsterdam 


\section{Contents}

Chapter 1: Introduction and outline of the thesis 7

Chapter 2: $\quad$ Two decades of axillary management in breast cancer 17

Chapter 3: "Axillary Reverse Mapping" Is visualisation of lymphatics 31 during axillary lymph node dissection clinically relevant?

Chapter 4: $\quad$ Axillary reverse mapping in axillary surgery for breast cancer - an update of the current status

Chapter 5: The clinical relevance of axillary reverse mapping (ARM): study protocol for a randomized controlled trial.

Chapter 6: $\quad$ Reliability of the Inverse Water Volumetry method to measure the volume of the upper limb

Chapter 7: Clinical utility of Axillary Reverse Mapping (ARM) in an era of changing perceptions concerning axillary surgery

Chapter 8: $\quad$ Axillary reverse mapping (ARM) in clinically node positive breast cancer patients

Chapter 9: Clinical impact of breast MRI with regard to axillary reverse mapping in clinically node positive breast cancer patients following neo-adjuvant chemotherapy

Chapter 10: Summary and general discussion

Chapter 11: Summary in Dutch / samenvatting in het Nederlands

Chapter 12: Valorization

Acknowledgements / dankwoord

List of publications 



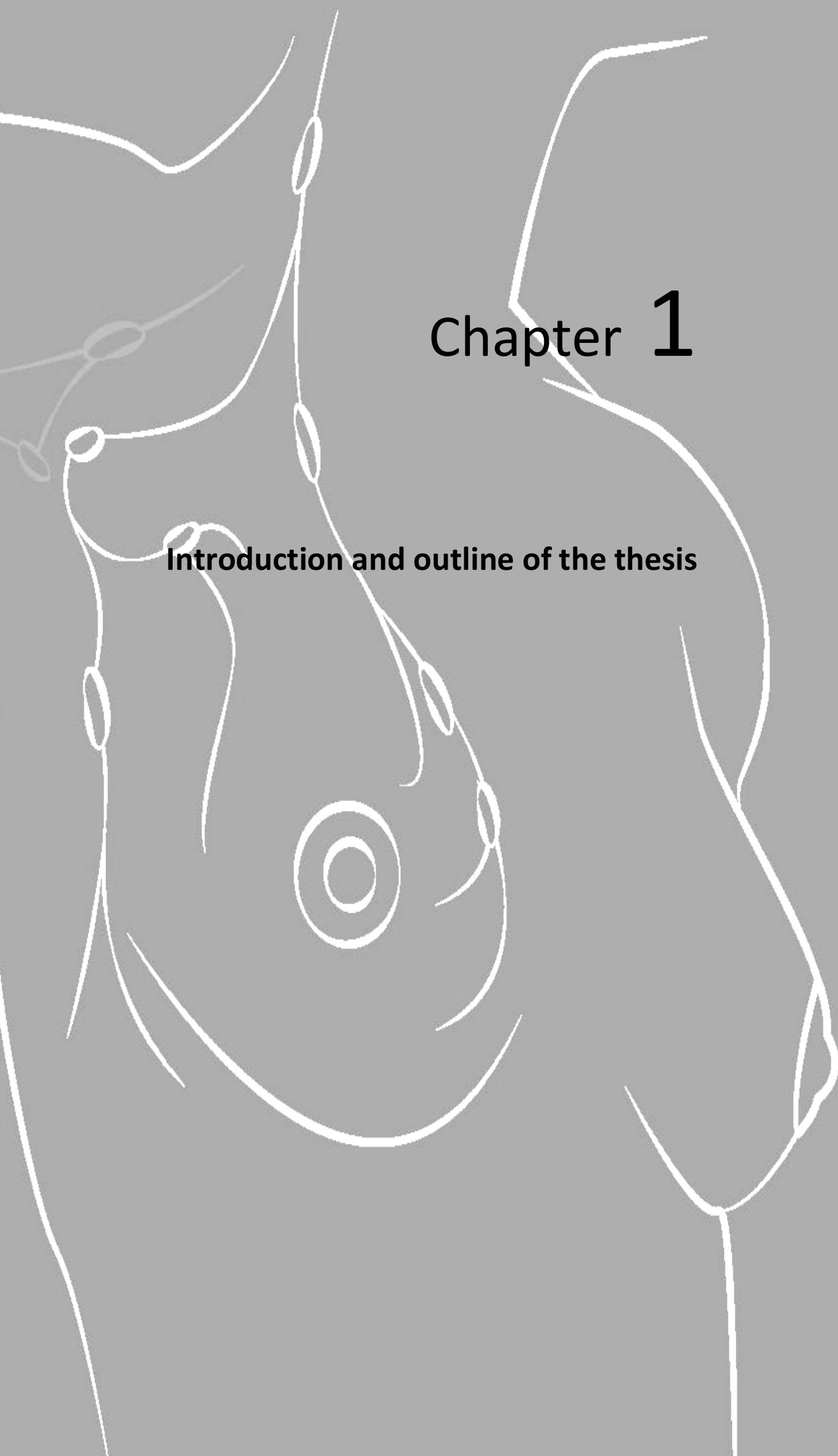




\section{Introduction}

\section{History of axillary lymph node dissection}

The first medical reports concerning breast cancer date back to the Egyptian era (3000 B.C.). From early on, surgery played a major role in the treatment of breast cancer, with the first surgical procedure ever reported being breast cauterization with a fire stick. ${ }^{1}$ Towards the end of the sixteenth century, the importance of the lymphatic system in the metastatic process of breast cancer was recognized and new surgical techniques were introduced. The Italian surgeon Marcus Aurelius Severinus (15801656) and the French surgeon Ambrose Paré (1510-1590) were possibly the first surgeons who mentioned the technique of axillary lymph node dissection (ALND) in patients with breast cancer. ${ }^{2}$

In 1894 William Halsted, professor of surgery at Johns Hopkins Hospital in Baltimore, United States, introduced the radical mastectomy, which became the gold standard in breast cancer surgery for the following 80 years. Halsted introduced the radical mastectomy which consisted of complete removal of the breast and all axillary and interpectoral lymph nodes, including resection of both pectoral muscles in a single en bloc procedure in an effort to prevent (lymphatic) spread of cancer. ${ }^{3}$ In the late sixties of the former century David Patey and John Madden developed a refinement of this technique by performing a modified radical mastectomy in order to reduce the postoperative morbidity of the Halstad radical mastectomy. Patey concluded that excision of the pectoralis major was not justified on pathological grounds. In this procedure all axillary and interpectoral lymph nodes (Level I-III) including the pectoralis minor muscle were resected. ${ }^{4}$ Madden advocated a slight modification of the technique by preserving both pectoralis muscles which still enables adequate access to axillary and interpectoral lymph nodes. ${ }^{5}$

By the end of the $20^{\text {th }}$ century surgeons became increasingly aware of the necessity to reduce morbidity, especially upper extremity lymphedema, induced by ALND. ${ }^{6-10}$ Upper extremity lymphedema is the most frequent complication following axillary surgery, apart from arm and/or shoulder dysfunction, pain and paresthesia. A major shift to less invasive axillary surgery was seen following the introduction of the sentinel lymph node biopsy (SLNB) in the late nineties. The concept of the sentinel lymph node (SLN) being predictive of the status of the regional lymphatic basin is commonly attributed to Ramon Cabanas, a South American surgeon, following his pioneering work on the lymphatic drainage in carcinoma of the penis. ${ }^{11}$ However, the first reported clinical study on SLNB appeared nearly 20 years prior to Cabanas's publication, when Ernest Gould and his colleagues from the Washington Hospital Centre published their work on the SLNB in tumours of the parotid gland. ${ }^{12}$ 
Giuliano et al. pioneered the use of SLNB in breast cancer patients. ${ }^{13}$ In 174 breast cancer patients, he was able to localize a SLN in 114 of them (66\%) and in 109 of these 114 patients (96\%) there was a positive correlation between the SLN status and the axillary nodal status. In this study the SLNB and ALND were performed during the same operation in order to ascertain the oncological safety of this less invasive axillary staging procedure. Subsequently, the results of the National Surgical Adjuvant Breast and Bowel Project (NSABP) B-32 trial, reported by Krag et al., demonstrated that completion ALND can be omitted in patients with a negative SLN. ${ }^{14}$ In this study 5611clinically node-negative patients were randomised to SLNB alone versus SLNB and completion ALND. Over an 8-year follow-up period, a significant decrease in morbidity was observed in the SLNB alone group and no significant differences were seen in overall survival, disease-free survival or loco-regional control of disease between the two groups. However, ALND remained the gold standard for SLN positive breast cancer patients.

A major step in the strategy towards less extensive axillary surgery was made in 2009. Based on clinical evidence including a study by Galimberti et al., the St. Gallen Consensus Panel recommended not to perform completion ALND in patients with micrometastases or isolated tumour cells demonstrated in the SLN. ${ }^{15,16}$

Recently, the results of the American College of Surgeons Oncology Group (ACOSOG) Z0011 trial, published in 2011, have even cast doubts on the necessity to perform completion ALND in patients with SLN macrometastases. ${ }^{17,18}$ This trial showed that omitting completion ALND does not result in inferior overall- and disease free survival in cT1-2NO breast cancer patients with macrometastases in one or two SLN(s) treated with breast-conserving therapy, including irradiation of the breast as well as adjuvant systemic therapy.

More recently, the results of the European Organisation for Research and Treatment of Cancer (EORTC) AMAROS trial, published in 2014, showed that, for patients with T1-2 primary breast cancer without palpable lymphadenopathy, axillary radiotherapy is not inferior compared to completion ALND, with regard to local regional control and disease-free survival, following a positive SLNB and is associated with less morbidity. ${ }^{19}$ Ever since publication of these recent studies there is an ongoing debate on the role of ALND in future management of the axilla. Nevertheless, ALND is still the gold standard in patients with clinically node-positive breast cancer and the treatment of first choice for a substantial number of patients with macrometastases found in the $\mathrm{SLN}$, for instance when mastectomy is accompanied by direct reconstruction with a tissue expander. ${ }^{8,20-23}$

Survival of breast cancer patients has been increasing in recent years, due to both use of more effective systemic therapy as well as earlier detection due to screening 
programs. ${ }^{24}$ Therefore, the number of patients potentially at risk for late effects (morbidity) of any axillary treatment is growing.

\section{Lymphedema}

The function of the lymphatic system is to drain excess fluid and overflowing substances, i.e. proteins, colloids and other molecules, which are too large to re-enter the blood compartment directly. The physiology of lymphedema is an imbalance between capillary filtration and uptake in both the lymphatic and microvascular system. Although initially lymphedema presents as a soft increase of the volume of the subcutaneous layer, irreversible structural changes resulting in interstitial fibrosis or atrophy will develop over a period of time. A distinction is made between two types of lymphedema. Primary lymphedema is either congenital or has an unknown cause, and secondary lymphedema comprises those cases for which a cause can be identified, such as infection, operation or radiotherapy. Lymphedema is characterized by gross swelling of the affected limb. Patients with lymphedema report a higher frequency of infections, pain, restriction of movement and a structurally lower quality of life. Furthermore, the total health care costs are much higher in patients with lymphedema compared to patients without lymphedema, mainly due to the treatment of complications and the increased need for physical therapy. ${ }^{25}$

Treatment of lymphedema is mainly based on non-invasive strategies, such as physical therapy and use of compression garments. Occasionally surgical treatment is performed. These conservative, non-invasive strategies may be helpful in some patients however they are time-consuming, expensive and only supportive. ${ }^{26,27}$ Surgical treatments such as lymphatico-lymphatic bypass (LLB) and lymphaticovenous anastomosis (LVA) have been reported to result in some reduction of volume but currently there is no definitive cure for lymphedema. Thus, prevention of lymphedema seems the best option. ${ }^{28}$

Postoperative incidence of upper extremity lymphedema following ALND ranges from $7 \%$ to $77 \% .^{9,29-35}$ This wide range in reported figures depends on whether ALND has been combined with subsequent axillary radiotherapy, length of follow-up and the different definitions used for upper extremity lymphedema. ${ }^{36,37}$ Furthermore, a difference in the measurement methods of upper extremity lymphedema is also to be accounted for. Commonly used methods to assess and to grade the presence of upper extremity lymphedema are: volume displacement measurement, arm circumference and questionnaires. ${ }^{9,31,38-42}$ The fact that there are so many measurement methods in different studies limits the ability to compare the postoperative incidence of upper extremity lymphedema. 


\section{Axillary Reverse Mapping (ARM)}

In 2007, the first reports concerning a procedure which enables discrimination of the upper extremity and breast lymphatic drainage in the axilla were presented; which is referred to as "axillary reverse mapping" (ARM) (Figure 1.1). ${ }^{43,44}$ The fundamental concept of the ARM procedure is based on the assumption that the upper extremity and breast each have their own discrete pathways of lymphatic drainage (Figure 1.2).

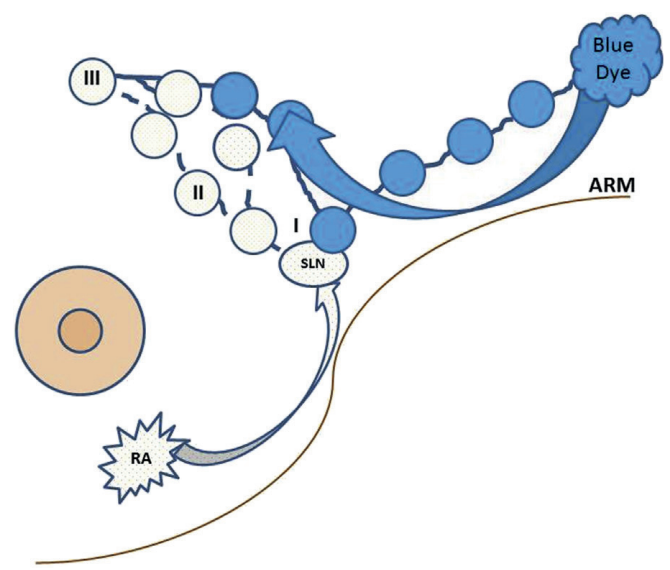

Figure 1.1 Axillary reverse mapping. ${ }^{45}$ (Ann Surg Oncol. 2007; with permission)

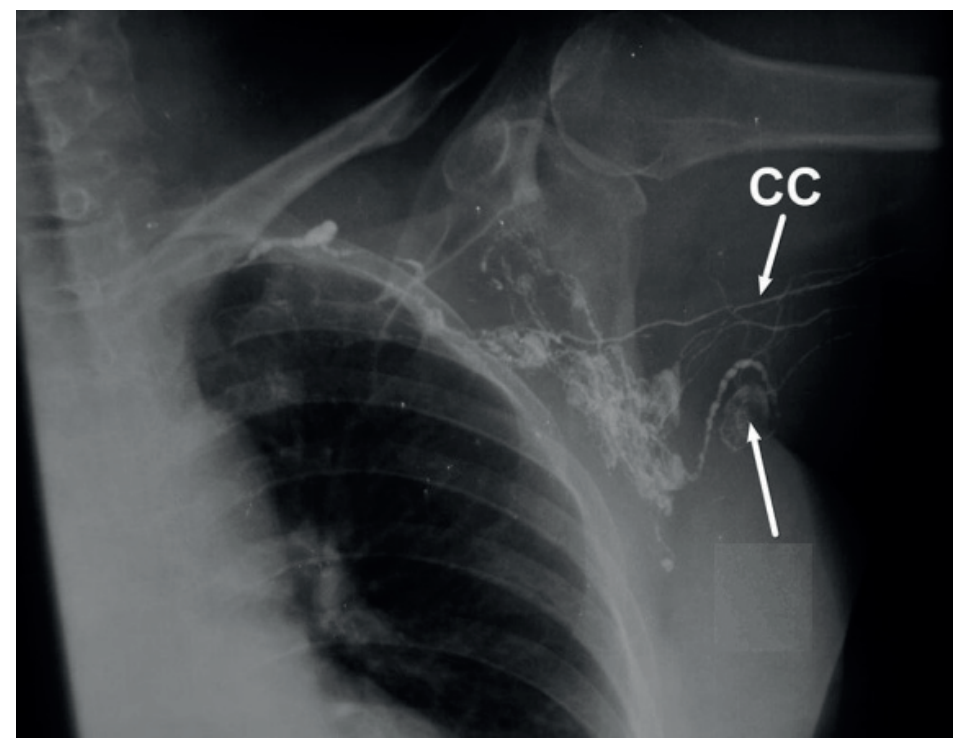

Figure 1.2 Pathways of lymphatic drainage, including SLN and ARM lymph node. ${ }^{46}$ (Eur J Surg Oncol. 2012 with permission) 
The procedure is called "reverse" due to the fact that the basic principle of the procedure is quite the opposite from its use during the SLNB. During the SLNB blue dye and radiolabelled colloid are used to visualize the SLN for selective removal. During the ARM procedure blue dye is injected to visualize upper extremity lymph nodes in order to preserve lymph nodes and lymphatics. This concept was tested and validated in a mouse model in which two different lymphatic pathways were demonstrated by a two-colour spectral fluorescence lymphangiography. ${ }^{47}$ If upper extremity lymphedema is caused by disruption of axillary lymphatics and/or removal of ARM lymph nodes, being able to identify and preserve them could possibly prevent upper extremity lymphedema during ALND or SLNB. Further research is needed to provide evidence for the clinical utility and safety of the ARM procedure and to select which breast cancer patients are candidates for this new procedure.

\section{Outline of the thesis}

This thesis describes the introduction of axillary reverse mapping (ARM); a procedure which enables discrimination of the upper extremity and breast lymphatic drainage in the axilla.

A major shift to less invasive axillary surgery was seen in the last 15 years. In chapter 2 we describe the changing patterns of care regarding the axilla, including the introduction of SLNB in the late 1990s, implementation of the results of the American College of Surgeons Oncology Group Z0011 study, and the initial effects of the European Organization for Research and Treatment of Cancer AMAROS study. For this study, data of the cancer registry of the former Comprehensive Cancer Centre South (currently the Netherlands Comprehensive Cancer Organisation) were used. One of the most important reasons to critically re-evaluate the need for ALND was the postoperative morbidity associated with this procedure.

In 2007 the ARM technique was described to reduce the incidence of upper extremity lymphedema in patients who undergo ALND. In chapter $\mathbf{3}$ we describe the possible clinical relevance of the ARM technique. In chapter 4 a review of the current literature discusses the feasibility, safety and relevance of ARM.

In chapter 5 we present the design of a randomized controlled multicentre trial. The aim of the trial (ARM study) is to determine the clinical relevance and safety of selectively sparing upper-limb axillary lymph nodes and their corresponding lymphatics by means of ARM. The primary outcome of this ongoing trial will be the presence of lymphedema in the upper limb. In this study upper extremity 
lymphedema will be measured using several measurement techniques as well as patient reported outcome. One of these methods, the water-displacement method, is regarded as the gold standard for the measurement of upper extremity lymphedema. For the assessment of arm volume, the "Inverse Water Volumetry apparatus" (IWVapparatus) will be used. In chapter 6 we describe the reliability and reproducibility of this method for measurement of arm volume.

Chapter 7 discusses the clinical utility of ARM in an era of changing perceptions concerning axillary surgery.

In chapter $\mathbf{8}$ we prospectively evaluate the incidence of metastatic involvement of ARM lymph nodes in clinical node-positive breast cancer patients who underwent ALND following neo-adjuvant chemotherapy (NAC), in comparison to patients who underwent ALND without NAC. In chapter 9 we retrospectively evaluated the incidence of metastatic involvement of ARM lymph nodes in clinically node-positive breast cancer patients with a radiological complete response of the axillary lymph nodes at breast MRI who underwent primary ALND following NAC, in comparison to patients without a radiological complete response of the axillary lymph nodes at breast MRI.

Chapter 10 provides a summary of this thesis, a general discussion and future perspectives, including several new initiatives and suggestions for additional clinical studies. Chapter 11 provides the summary and discussion in Dutch. 


\section{References}

1. Mansfield CM. Early breast cancer its history and results of treatment. Exp Biol Med. 1976;5:1-129.

2. Robinson JO. Treatment of breast cancer through the ages. Am J Surg. 1986;151:317-33.

3. Halsted WS. I. The Results of Operations for the Cure of Cancer of the Breast Performed at the Johns Hopkins Hospital from June, 1889, to January, 1894. Ann Surg. 1894;20:497-555.

4. Patey DH. A review of 146 cases of carcinoma of the breast operated on between 1930 and $1943 . \mathrm{Br} \mathrm{J}$ Cancer. 1967;21:260-9.

5. Madden JL. Modified radical mastectomy. Surg Gynecol Obstet. 1965;121:1221-30.

6. Ivens D, Hoe AL, Podd TJ, Hamilton CR, Taylor I, Royle GT. Assessment of morbidity from complete axillary dissection. Br J Cancer. 1992;66:136-8.

7. Liljegren G, Holmberg L. Arm morbidity after sector resection and axillary dissection with or without postoperative radiotherapy in breast cancer stage I. Results from a randomised trial. Uppsala-Orebro Breast Cancer Study Group. Eur J Cancer. 1997;33:193-9.

8. Noguchi M, Miwa K, Michigishi T, Yokoyama K, Nishijima H, Takanaka T, et al. The Role of Axillary Lymph Node Dissection in Breast Cancer Management. Breast Cancer. 1997;4:143-53.

9. Schijven MP, Vingerhoets AJ, Rutten HJ, Nieuwenhuijzen GA, Roumen RM, van Bussel ME, et al. Comparison of morbidity between axillary lymph node dissection and sentinel node biopsy. Eur J Surg Oncol. 2003;29:341-50.

10. Schunemann $\mathrm{H}$, Willich $\mathrm{N}$. Lymphoedema of the arm after primary treatment of breast cancer. Anticancer Res. 1998;18:2235-6.

11. Cabanas RM. An approach for the treatment of penile carcinoma. Cancer. 1977;39:456-66.

12. Gould EA, Winship T, Philbin PH, Kerr HH. Observations on a "sentinel node" in cancer of the parotid. Cancer. 1960;13:77-8.

13. Giuliano AE, Kirgan DM, Guenther JM, Morton DL. Lymphatic mapping and sentinel lymphadenectomy for breast cancer. Ann Surg. 1994;220:391-8.

14. Krag DN, Anderson SJ, Julian TB, Brown AM, Harlow SP, Ashikaga T, et al. Technical outcomes of sentinel-lymph-node resection and conventional axillary-lymph-node dissection in patients with clinically node-negative breast cancer: results from the NSABP B-32 randomised phase III trial. Lancet Oncol. 2007;8:881-8.

15. Goldhirsch A, Ingle JN, Gelber RD, Coates AS, Thurlimann B, Senn HJ, et al. Thresholds for therapies: highlights of the St Gallen International Expert Consensus on the primary therapy of early breast cancer 2009. Ann Oncol. 2009;20:1319-29.

16. Galimberti V. Axillary sentinel lymph node: How low can you go? Breast 2009;18 (Suppl 1):S12 (Abstr S29).

17. Giuliano AE, Hunt KK, Ballman KV, Beitsch PD, Whitworth PW, Blumencranz PW, et al. Axillary dissection vs no axillary dissection in women with invasive breast cancer and sentinel node metastasis: a randomized clinical trial. JAMA. 2011;305:569-75.

18. Giuliano AE, McCall L, Beitsch P, Whitworth PW, Blumencranz P, Leitch AM, et al. Locoregional recurrence after sentinel lymph node dissection with or without axillary dissection in patients with sentinel lymph node metastases: the American College of Surgeons Oncology Group Z0011 randomized trial. Ann Surg. 2010;252:426-32.

19. Donker M, van Tienhoven G, Straver ME, Meijnen P, van de Velde CJ, Mansel RE, et al. Radiotherapy or surgery of the axilla after a positive sentinel node in breast cancer (EORTC 10981-22023 AMAROS): a randomised, multicentre, open-label, phase 3 non-inferiority trial. Lancet Oncol. 2014;15:1303-10.

20. Tausch C, Baege A, Dietrich D, Vergin I, Heuer H, Heusler RH, et al. Can axillary reverse mapping avoid lymphedema in node positive breast cancer patients? Eur J Surg Oncol. 2013;39:880-6.

21. Noguchi M, Morioka E, Ohno Y, Noguchi M, Nakano Y, Kosaka T. The changing role of axillary lymph node dissection for breast cancer. Breast Cancer. 2013;20:41-6.

22. NOABON. Landelijke richtlijn mammacarcinoom 2.0. wwwoncolinenl/mammacarcinoom. 
23. Gross E, Hannoun-Levi JM, Rouanet P, Houvenaeghel G, Teissier E, Ellis S, et al. [Evaluation of immediate breast reconstruction and radiotherapy: factors associated with complications]. Cancer Radiother. 2010;14:704-10.

24. Louwman WJ, Voogd AC, van Dijck JA, Nieuwenhuijzen GA, Ribot J, Pruijt JF, et al. On the rising trends of incidence and prognosis for breast cancer patients diagnosed 1975-2004: a long-term populationbased study in southeastern Netherlands. Cancer Causes Control. 2008;19:97-106.

25. Shih YC, Xu Y, Cormier JN, Giordano S, Ridner SH, Buchholz TA, et al. Incidence, treatment costs, and complications of lymphedema after breast cancer among women of working age: a 2-year follow-up study. J Clin Oncol. 2009;27:2007-14.

26. Nagase T, Gonda K, Inoue K, Higashino T, Fukuda N, Gorai K, et al. Treatment of lymphedema with lymphaticovenular anastomoses. Int J Clin Oncol. 2005;10:304-10.

27. Torrisi JS, Joseph WJ, Ghanta S, Cuzzone DA, Albano NJ, Savetsky IL, et al. Lymphaticovenous bypass decreases pathologic skin changes in upper extremity breast cancer-related lymphedema. Lymphat Res Biol. 2015;13:46-53.

28. Finnane A, Janda M, Hayes SC. Review of the evidence of lymphedema treatment effect. Am J Phys Med Rehabil. 2015;94:483-98.

29. Blanchard DK, Donohue JH, Reynolds C, Grant CS. Relapse and morbidity in patients undergoing sentinel lymph node biopsy alone or with axillary dissection for breast cancer. Arch Surg. 2003;138:482-7.

30. Haid A, Koberle-Wuhrer R, Knauer M, Burtscher J, Fritzsche H, Peschina W, et al. Morbidity of breast cancer patients following complete axillary dissection or sentinel node biopsy only: a comparative evaluation. Breast Cancer Res Treat. 2002;73:31-6.

31. Leidenius $\mathrm{M}$, Leivonen $\mathrm{M}$, Vironen J, von Smitten $\mathrm{K}$. The consequences of long-time arm morbidity in node-negative breast cancer patients with sentinel node biopsy or axillary clearance. J Surg Oncol. 2005;92:23-31.

32. Mansel RE, Fallowfield L, Kissin M, Goyal A, Newcombe RG, Dixon JM, et al. Randomized multicenter trial of sentinel node biopsy versus standard axillary treatment in operable breast cancer: the ALMANAC Trial. J Natl Cancer Inst. 2006;98:599-609.

33. Ronka R, von Smitten $\mathrm{K}$, Tasmuth $\mathrm{T}$, Leidenius $\mathrm{M}$. One-year morbidity after sentinel node biopsy and breast surgery. Breast. 2005;14:28-36.

34. Schrenk P, Rieger R, Shamiyeh A, Wayand W. Morbidity following sentinel lymph node biopsy versus axillary lymph node dissection for patients with breast carcinoma. Cancer. 2000;88:608-14.

35. Swenson KK, Nissen MJ, Ceronsky C, Swenson L, Lee MW, Tuttle TM. Comparison of side effects between sentinel lymph node and axillary lymph node dissection for breast cancer. Ann Surg Oncol. 2002;9:745-53.

36. Coen JJ, Taghian AG, Kachnic LA, Assaad SI, Powell SN. Risk of lymphedema after regional nodal irradiation with breast conservation therapy. Int J Radiation Oncol Biol Phys. 2003;55:1209-15.

37. Johnson KC, Kennedy AG, Henry SM. Clinical Measurements of Lymphedema. Lymphat Res Biol. 2014;12:216-21.

38. Bulley C, Coutts F, Blyth C, Jack W, Chetty U, Barber M, et al. A Morbidity Screening Tool for identifying fatigue, pain, upper limb dysfunction and lymphedema after breast cancer treatment: a validity study. Eur J Oncol Nurs. 2014;18:218-27.

39. Barranger E, Dubernard G, Fleurence J, Antoine M, Darai E, Uzan S. Subjective morbidity and quality of life after sentinel node biopsy and axillary lymph node dissection for breast cancer. J Surg Oncol. 2005;92:17-22.

40. Kaulesar Sukul DM, den Hoed PT, Johannes EJ, van Dolder R, Benda E. Direct and indirect methods for the quantification of leg volume: comparison between water displacement volumetry, the disk model method and the frustum sign model method, using the correlation coefficient and the limits of agreement. J Biomed Eng. 1993;15:477-80.

41. Kettle JH, Rundle FF, Oddie TH. Measurement of upper limb volumes: a clinical method. Aus N Z J Surg. 1958;27:263-70.

42. Stanton AW, Badger C, Sitzia J. Non-invasive assessment of the lymphedematous limb. Lymphology. 2000;33:122-35. 
43. Nos C, Lesieur B, Clough KB, Lecuru F. Blue dye injection in the arm in order to conserve the lymphatic drainage of the arm in breast cancer patients requiring an axillary dissection. Ann Surg Oncol. 2007;14:2490-6.

44. Thompson M, Korourian S, Henry-Tillman R, Adkins L, Mumford S, Westbrook KC, et al. Axillary reverse mapping (ARM): a new concept to identify and enhance lymphatic preservation. Ann Surg Oncol. 2007;14:1890-5.

45. Ochoa D, Korourian S, Boneti C, Adkins L, Badgwell B, Klimberg VS. Axillary reverse mapping: five-year experience. Surgery. 2014;156:1261-8.

46. Pavlista D, Eliska O. Analysis of direct oil contrast lymphography of upper limb lymphatics traversing the axilla -- a lesson from the past -- contribution to the concept of axillary reverse mapping. Eur J Surg Oncol. 2012;38:390-4.

47. Hama Y, Koyama Y, Urano Y, Choyke PL, Kobayashi H. Simultaneous two-color spectral fluorescence lymphangiography with near infrared quantum dots to map two lymphatic flows from the breast and the upper extremity. Breast Cancer Res Treat. 2007;103:23-8. 


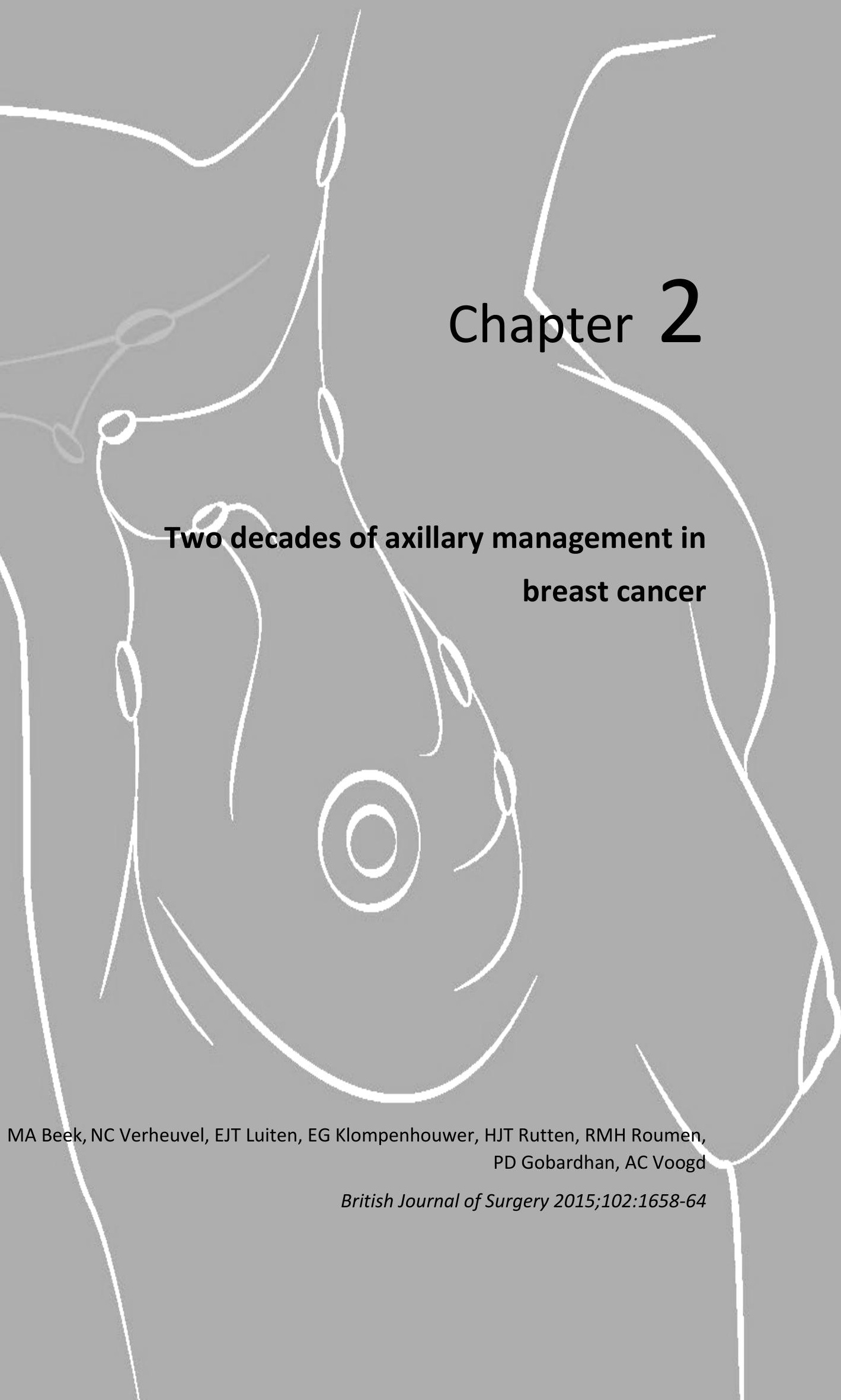




\section{Abstract}

\section{Background}

Axillary lymph node dissection (ALND) in patients with breast cancer provides prognostic information. For many years, positive nodes were the most important indication for adjuvant systemic therapy. It was also believed that regional control could not be achieved without axillary clearance in a positive axilla. However, during the past 20 years the treatment and staging of the axilla has undergone many changes. This large population-based study was conducted in the south-east of the Netherlands to evaluate the changing patterns of care regarding the axilla, including the introduction of sentinel lymph node biopsy (SLNB) in the late 1990s, implementation of the results of the American College of Surgeons Oncology Group Z0011 study, and the initial effects of the European Organization for Research and Treatment of Cancer AMAROS study.

\section{Methods}

Data from the population-based Eindhoven Cancer Registry of all women diagnosed with invasive breast cancer in the south of the Netherlands between January 1993 and July 2014 were used.

\section{Results}

The proportion of 34037 women staged by SLNB without completion ALND increased from $0 \%$ in $1993-1994$ to $69.0 \%$ in $2013-2014$. In the same period the proportion undergoing ALND decreased from $88.8 \%$ to $18.7 \%$. Among women with one to three positive lymph nodes, the proportion undergoing SLNB alone increased from $10.6 \%$ in 2011-2012 to $37.6 \%$ in 2013-2014.

\section{Conclusion}

This population-based study demonstrated the radical transformation in management of the axilla since the introduction of SLNB and following the recent publication of trials on management of the axilla with a low metastatic burden. 


\section{Introduction}

Axillary lymph node dissection (ALND) in patients with breast cancer has been performed for disease control, tumour staging, and to aid in decision-making regarding the use of adjuvant systemic treatment. ${ }^{1,2}$ An important reason for critical re-evaluation of the need for ALND was the postoperative morbidity associated with the procedure..$^{3-5}$ In the late 1990 s this led to the introduction of the sentinel lymph node biopsy (SLNB), ${ }^{6-9}$ which has become the standard of care in clinically nodenegative early-stage breast cancer. ${ }^{10-13}$ The SLNB was considered to be reliable for staging of the axilla and for the decision to omit ALND in patients with a negative sentinel lymph node (SLN).

The results of the American College of Surgeons Oncology Group (ACOSOG) Z0011 trial, published in 2011, have cast doubt on the need to perform completion ALND in patients with SLN metastases. ${ }^{14,15}$ This trial showed that omitting ALND does not result in inferior overall and disease-free survival rates in patients with cT1-2 NO breast cancer, with one or two SLN metastases treated with breast-conserving therapy as well as adjuvant chemotherapy.

Recently, the results of the European Organization for Research and Treatment of Cancer (EORTC) AMAROS (After Mapping of the Axilla: Radiotherapy or Surgery) trial, ${ }^{16}$ published in 2014, showed that both completion ALND and axillary radiotherapy after a positive SLN procedure provided excellent and comparable axillary control for patients with T1-2 primary breast cancer and no palpable lymphadenopathy.

The aim of the present study, using a large population-based registry, was to evaluate patterns of care in treatment of the axilla with regard to the introduction of the SLNB in 1998, and the impact of the ACOSOG Z0011 and EORTC AMAROS trials on management of the axilla in daily clinical practice.

\section{Methods}

Data of women from the Eindhoven region were retrieved from the Comprehensive Cancer Centre South Registry, which is part of the Dutch Cancer Registry and records data on all patients newly diagnosed with cancer in the south-east region of the Netherlands, an area with approximately 2.4 million inhabitants. Data were derived from ten hospitals: six large non-university teaching and four community hospitals. Data on patient and tumour characteristics, and treatment were collected based on 
the pathology reports and medical records. The patients were staged according to the TNM system of the International Union Against Cancer (UICC). ${ }^{17}$ Data were revised continuously to ensure completeness and accuracy.

To perform a truly population-based analysis, all women diagnosed with primary invasive breast cancer in the period from January 1993 to July 2014 were included in the analyses.

Patterns of care for the axilla were studied for the group as a whole, and also according to age group and pathological axillary node status ( $\mathrm{pN}$ category). The following age groups were defined: less than 50 years, 50-69 years, 70-79 years or 80 years or above. The following subgroups were defined according to $\mathrm{pN}$ category: pNO, patients without lymph node metastases; $\mathrm{pNO}(\mathrm{i}+)$, patients with isolated tumour cells (metastases smaller than $0.2 \mathrm{~mm}$ ); pN1mi, patients with micrometastases (metastases of $0.2-2.0 \mathrm{~mm}$ in size); pN1a, patients with metastases in one to three axillary lymph nodes, including at least one larger than $2 \mathrm{~mm} ; \mathrm{pN}>1 \mathrm{a}$, patients with metastases in more than three axillary lymph nodes or macrometastases in either supraclavicular and/or internal mammary lymph nodes.

Patients with pN1a disease were further subdivided with regard to type of breast surgery: mastectomy or breast-conserving surgery. Isolated tumour cells have been documented by the Dutch Cancer Registry only since 2003, following publication of the revised TNM system in $2002 .{ }^{17}$

\section{Statistical analysis}

The $\chi^{2}$ test was used to determine whether observed trends in axillary management were statistically significant. Statistical analyses were performed using SPSS ${ }^{\circledR}$ version 21.0 (IBM, Armonk, New York, USA). P values less than 0.05 were considered significant.

\section{Results}

Between January 1993 and July 2014, a total of 34037 women were treated for primary invasive breast cancer in the south-east region of the Netherlands. Baseline characteristics are shown in Table 2.1. Overall, $51.8 \%$ of the women had breastconserving surgery, $39.4 \%$ had a mastectomy, and $8.8 \%$ did not undergo any definitive breast surgery or the type of surgery was not documented properly. 
Table 2.1 Characteristics at diagnosis of patients treated for primary breast cancer in south-east Netherlands, 1993-2014

\begin{tabular}{|c|c|}
\hline & No. of patients ( $n=34037$ ) \\
\hline \multicolumn{2}{|l|}{ Age (years) } \\
\hline$<50$ & $8217(24.1)$ \\
\hline $50-69$ & $16514(48.5)$ \\
\hline$\geq 70$ & $9306(27.3)$ \\
\hline \multicolumn{2}{|l|}{ Period of diagnosis } \\
\hline 1993-1994 & $2455(7.2)$ \\
\hline 1995-1996 & $2386(7.0)$ \\
\hline 1997-1998 & $2640(7.8)$ \\
\hline 1999-2000 & $2821(8.3)$ \\
\hline 2001-2002 & $3095(9.1)$ \\
\hline 2003-2004 & $3382(9.9)$ \\
\hline 2005-2006 & $3303(9.7)$ \\
\hline 2007-2008 & $3568(10.5)$ \\
\hline 2009-2010 & $3640(10.7)$ \\
\hline 2011-2012 & $3883(11.4)$ \\
\hline 2013-2014 & $2864(8.4)$ \\
\hline \multicolumn{2}{|l|}{ Tumour category } \\
\hline pT1 & $19111(56.1)$ \\
\hline pT2 & 11199 (32.9) \\
\hline pT3 & $1200(3.5)$ \\
\hline pT4 & $1724(5.1)$ \\
\hline Not known & $803(2.4)$ \\
\hline \multicolumn{2}{|l|}{ Node category } \\
\hline pNO & $18181(53.4)$ \\
\hline $\mathrm{pNO}(\mathrm{i}+)$ & $522(1.5)$ \\
\hline $\mathrm{pNmi}$ & $1326(3.9)$ \\
\hline $\mathrm{pN} 1 \mathrm{a}$ & $7074(20.8)$ \\
\hline $\mathrm{pN}>1 \mathrm{a}$ & $2763(8.1)$ \\
\hline Not known & $4171(12.3)$ \\
\hline \multicolumn{2}{|l|}{ Histology } \\
\hline Ductal & $26763(78.6)$ \\
\hline Lobular mixed & $5159(15.2)$ \\
\hline Other & $1378(4.0)$ \\
\hline Unknown & $737(2.2)$ \\
\hline \multicolumn{2}{|l|}{ Type of surgery } \\
\hline Breast-conserving surgery & $17636(51.8)$ \\
\hline Mastectomy & 13416 (39.4) \\
\hline None/unknown & $2985(8.8)$ \\
\hline \multicolumn{2}{|l|}{ Type of axillary staging } \\
\hline None & $3685(10.8)$ \\
\hline ALND only & 13421 (39.4) \\
\hline SLNB only & 12351 (36.3) \\
\hline SLNB + ALND & 4396 (12.9) \\
\hline Not known & $184(0.5)$ \\
\hline
\end{tabular}

Values in parentheses are percentages. pNO(i+), isolated tumour cells; pNmi, micrometastases; ALND, axillary lymph node dissection; SLNB, sentinel lymph node biopsy. 
In 1997-1998, SLNB was used for the first time in patients treated in the Eindhoven region (Figure 2.1). In this period, a total of 2621 women were treated for primary invasive breast cancer, of whom $80(3.1 \%)$ had staging of the axilla with SLNB alone and 229 (8.7\%) with SLNB followed by completion ALND. In 2013-2014, a total of 2843 women were treated for primary invasive breast cancer, of whom 1961 (69.0\%) had axillary staging with SLNB alone and 192 (6.8\%) with SLNB plus completion ALND. The proportion of women without axillary staging (no ALND or SLNB) ranged from $11.2 \%$ in $1993-1994$ to $12.4 \%$ in $2013-2014$ (Figure 2.1).
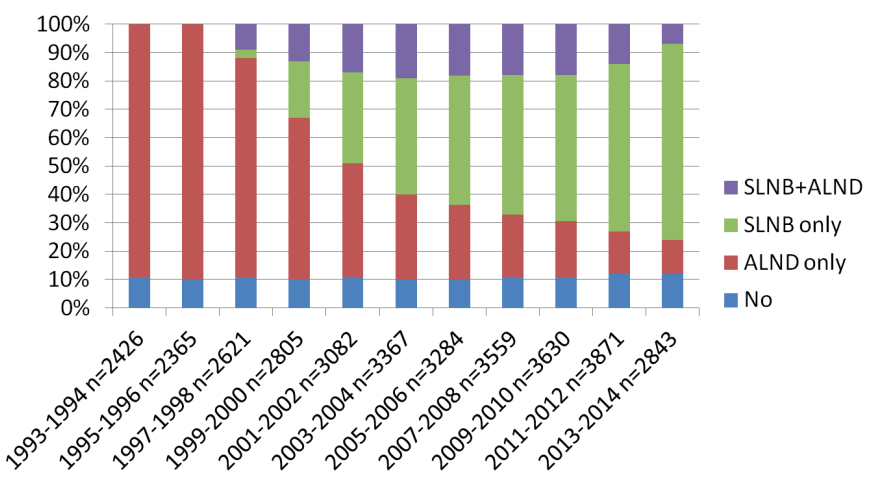

Figure 2.1 Trend in axillary staging and management for all patients, 1993-2014. Values in parentheses are numbers of patients. SLNB, sentinel lymph node biopsy; ALND, axillary lymph node dissection.

Among women aged 50 years or less, the proportion staged with SLNB alone increased from 17.7 per cent in 1999-2000 to 64.3\% in 2013-2014 (Figure 2.2A). A similar trend was observed in the age groups of 50-69 and 70-79 years (Figure 2.2B,C). In contrast to the other three groups, the proportion of women aged 80 years or above who did not undergo axillary staging increased from $45.3 \%$ in 1993-1994 to $56.8 \%$ in 2013-2014 (Figure 2.2D). In this age group, the proportion undergoing ALND decreased from $54.7 \%$ in $1993-1994$ to $9.8 \%$ in $2013-2014$. 

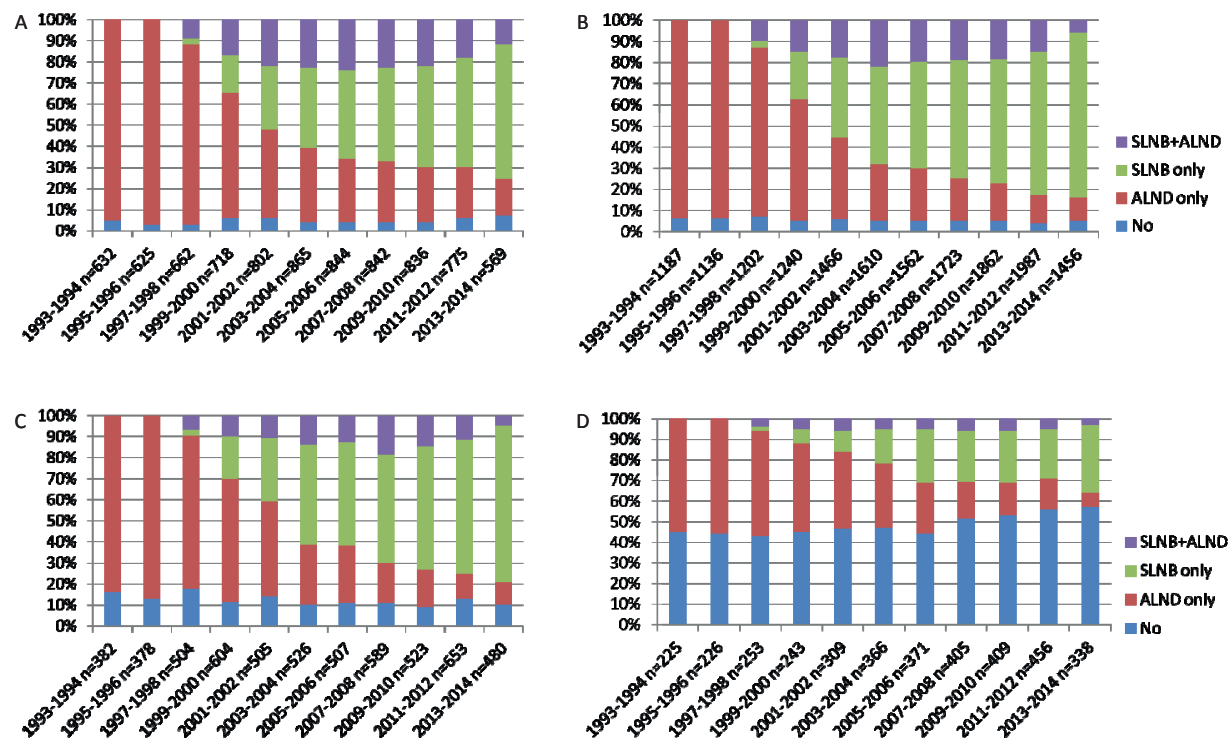

Figure 2.2 Trend in axillary staging and management, 1993-2014, in patients aged: A less than 50 years, B 50-69 years, $\mathbf{C} 70-79$ years and $\mathbf{D} 80$ years or more.

Among women with pNO disease, the proportion staged with ALND decreased from $100 \%$ in $1993-1994$ to $6.1 \%$ in 2013-2014 (Figure 2.3A).

The proportion of patients with isolated tumour cells increased from $11.3 \%$ (59 of 521 ) in 2003-2004 to $22.8 \%$ (119 of 521) in 2013-2014. In 2003-2004, 47.4\% of the women diagnosed with isolated tumour cells by SLNB underwent completion ALND; by 2013-2014 this proportion had decreased to $1.7 \%$ (Figure $2.3 \mathrm{~B}$ ).

The proportion of women with micrometastases increased from 1.3\% (17 of 1321) in 1993-1994 to 12.0\% (159 of 1321) in 2013-2014. The use of SLNB alone in women with micrometastases increased from $34.9 \%$ in $1999-2000$ to $86.2 \%$ in $2013-2014$. Until 2012, most patients with micrometastases demonstrated by SLNB also underwent completion ALND; by 2013-2014 this proportion had decreased to $6.9 \%$ (Figure 2.3C). 

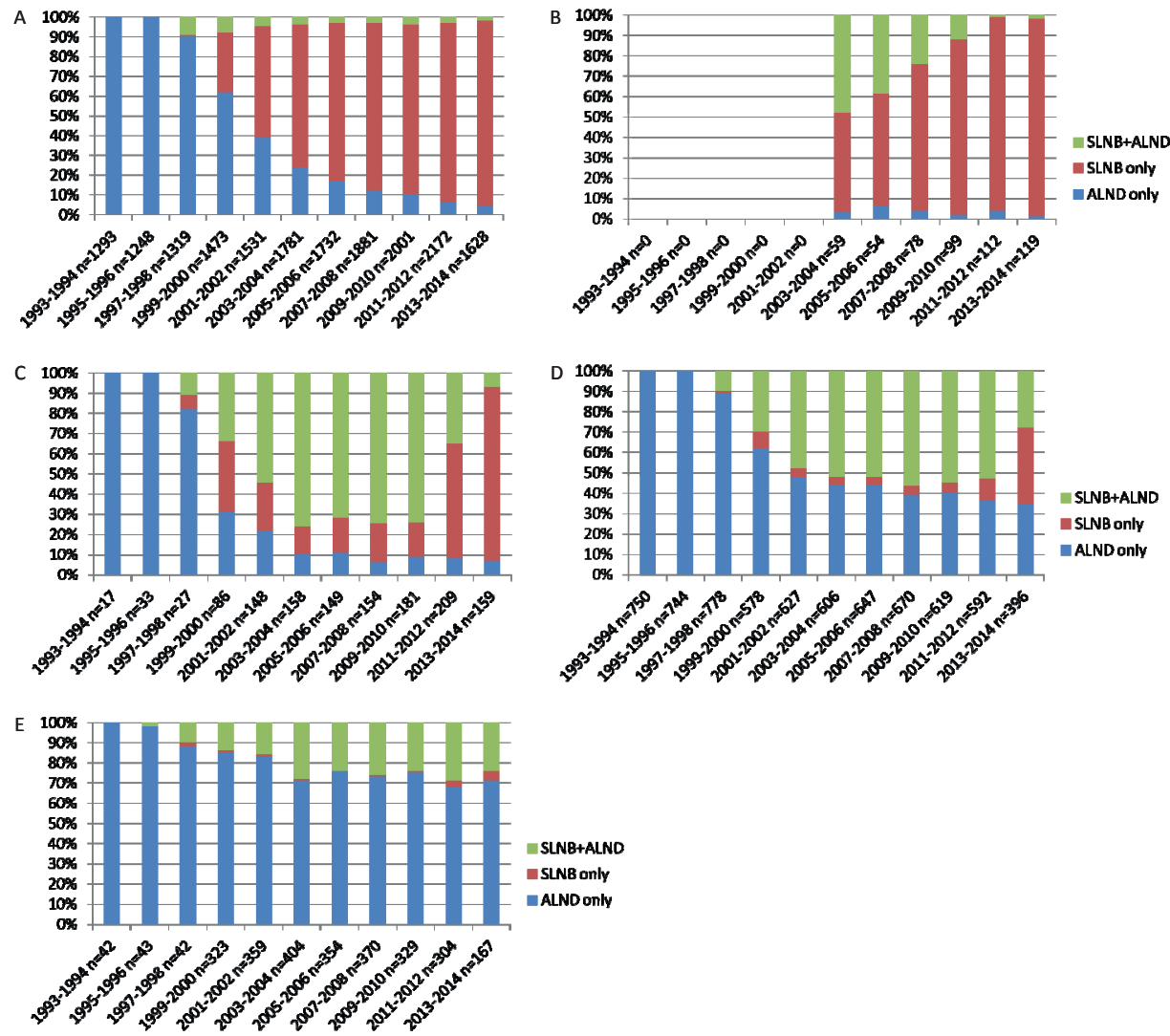

Figure 2.3 Trend in axillary staging and management, 1993-2014, in patients with: A no axillary metastasis ( $\mathrm{pNO}$ ), B isolated tumour cells (metastases smaller than $0.2 \mathrm{~mm}, \mathrm{pNO}(\mathrm{i}+)$ ), C micrometastases (metastases between 0.2 and $2.0 \mathrm{~mm}, \mathrm{pN} 1 \mathrm{mi}$ ), D metastases in one to three axillary lymph nodes, including at least one larger than $2 \mathrm{~mm}$ (pN1a), E more than three axillary lymph nodes or macrometastases in either supraclavicular and/or internal mammary lymph nodes $(\mathrm{pN}>1 \mathrm{a})$. Values in parentheses are numbers of patients. SLNB, sentinel lymph node biopsy; ALND, axillary lymph node dissection.

Between 1999 and 2011, about 5\% of women with pN1a disease were staged by SLNB alone, compared with $37.6 \%$ of those diagnosed in 2013-2014 (Figure 2.3D). When analysed according to type of surgery, in 2013-2014 the proportion of women undergoing breast-conserving surgery who had SLNB alone was $49.6 \%$, compared with $22.4 \%$ of women having a mastectomy (Figure $2.4 \mathrm{~A}, \mathrm{~B}$ ).

Among women with $\mathrm{pN}>1$ a disease, the proportion who had SLNB alone was $5.4 \%$ in 2013-2014 versus 3\% or less before that period (Figure 2.3E). 
All time trends in axillary staging described above were statistically significant $(P<0.001)$. Of 110 women with one to three positive axillary lymph nodes who had breast-conserving surgery and SLNB without completion ALND in 2013-2014, 108 $(98.2 \%)$ received adjuvant systemic treatment. In addition, of the 39 women with one to three positive axillary lymph nodes who had a mastectomy and SLNB without completion ALND in the same period, 32 (82\%) underwent postmastectomy radiotherapy.
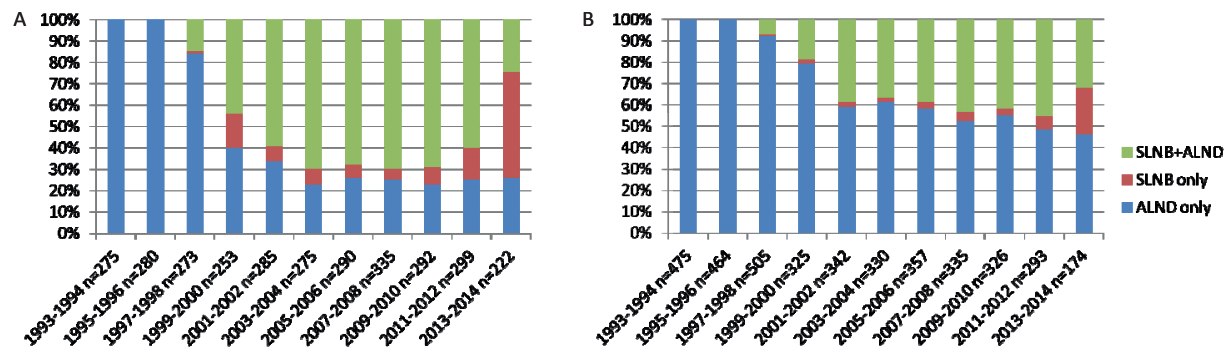

Figure 2.4 Trend in axillary staging and management, 1993-2014, in patients with one to three axillary lymph node metastases ( $\mathrm{pN1} 1 \mathrm{a}$ ) undergoing a breast-conserving surgery or b mastectomy. Values in parentheses are numbers of patients. SLNB, sentinel lymph node biopsy; ALND, axillary lymph node dissection

\section{Discussion}

In this large population-based study, the patterns of axillary staging based on a large and unselected patient population are presented. A major shift to less invasive axillary lymph node surgery was found over an interval of almost two decades.

SLNB was first introduced in Dutch national breast cancer treatment guidelines in 1999. ${ }^{18,19}$ Currently, SLNB is used in more than $70 \%$ of all women with breast cancer. As a consequence of the implementation of SLNB, the proportion of women undergoing primary ALND has decreased. This replacement of ALND by SLNB has been described previously in several studies. ${ }^{18,20,21}$

Before publication of the results of the ACOSOG Z0011 trial, the finding of one or more positive SLNs was a clear indication for completion ALND. The situation was always less distinct when the SLN contained micrometastases or isolated tumour cells and no macrometastatic disease. ${ }^{22,23}$ The debate on the necessity of performing completion ALND in these patients is reflected by the treatment patterns observed in the present study, and has been described in more detail previously. ${ }^{23}$ In 2009 , the St 
Gallen Consensus Panel recommended that completion ALND should not be performed in patients with micrometastases or isolated tumour cells in the SLN. ${ }^{24,25}$ According to the present data, in 2013-2014 more than $85 \%$ of the women with SLN micrometastases were staged by SLNB alone, and for women with SLN isolated tumour cells this was almost $100 \%$.

Although the ACOSOG Z0011 trial has been criticized for several reasons, including a heavy selection of patients, too many participating centres with low accrual rates, and only half of the initially planned 1900 patients randomized, publication of its results in 2011 can be considered as a practice-changing event in the management of the axilla. The findings from this trial questioned the need for completion ALND in patients with SLNB metastases. ${ }^{14}$ By also including the periods 2011-2012 and 2013-2014 in the present study, comparison of the situation before and after the publication of the ACOSOG Z0011 trial can be made. It is clear that axillary staging radically changed for patients with pN1a disease, even though the results of the Z0011 trial had not been integrated in the Dutch breast cancer treatment guidelines. In 2013-2014, 37.6\% of these women had axillary staging by SLNB alone, compared with about $5 \%$ in 2009-2010. This difference was even more pronounced when the type of surgery was taken into account. Before publication of the ACOSOG Z0011 trial, 7.9\% of patients treated with breast-conserving surgery (one of the main inclusion criteria of the ACOSOG Z0011 trial) were staged by SLNB alone, compared with $49.6 \%$ in 2013-2014.

The clinical impact of the ACOSOG Z0011 trial has been evaluated previously in several other studies. Wright and colleagues ${ }^{26}$ performed a retrospective population-based study to identify the impact of the ACOSOG Z0011 trial on breast cancer surgery in a community hospital setting. In this study significant changes in practice over time in the post- ACOSOG Z0011 trial era, including reduced rates of completion ALND, were observed. The rate of completion ALND in patients meeting ACOSOG Z0011 criteria decreased from $90 \%$ to $29 \%$. A limitation of this population-based study, compared with the present study, was its relatively small number of patients (1125). A singlecentre study by Caudle and co-workers ${ }^{27}$ found a decrease in the rate of a completion ALND from $85 \%$ in the years before publication of the ACOSOG Z0011 results to $24 \%$ thereafter in patients with a positive SLN who met the ACOSOG Z0011 eligibility criteria. Caretta-Weyer et al. ${ }^{28}$ suggested that a completion ALND could be spared in $38 \%$ of their patients, whereas Dengel and colleagues ${ }^{29}$ showed that completion ALND could be avoided in $84 \%$ of patients having breast-conserving surgery. Two other single-institution studies ${ }^{30,31}$ have also reported rates of approximately $45 \%$. In another single-centre study, Robison and co-workers, ${ }^{32}$ using a prospectively collected database of patients with CT1-2 NO breast cancer, found that before publication of the ACOSOG Z0011 trial $84 \%$ of all patients with macrometastases discovered during SLNB underwent completion ALND, compared with 63\% afterwards. 
Gainer and colleagues ${ }^{33}$ and Massimino et al. ${ }^{34}$ used questionnaire surveys to evaluate the impact of the ACOSOG Z0011 trial on surgical practice. Gainer and co-workers asked 2759 members of the American Society of Breast Surgeons to complete a questionnaire regarding the practice type and duration, familiarity with ACOSOG Z0011, and mode of receiving the trial results. The majority $(97 \%)$ of respondents indicated familiarity with the ACOSOG Z0011 trial, and many had incorporated it into their practice. Massimino and colleagues reported a $63 \%$ decrease in completion ALND for patients in the Pacific north-west, similar to the patients in the ACOSOG Z0011 trial.

Recently, the EORTC AMAROS trial ${ }^{16}$ and the International Breast Cancer Study Group (IBCSG) trial $^{35}$ challenged the need for completion ALND in patients with SLN metastases. The AMAROS trial compared completion ALND with axillary radiotherapy in patients with a positive SLN, T1-2 primary breast cancer and no palpable lymphadenopathy, and showed that excellent axillary control can be achieved with radiotherapy. Before publication of the results of the AMAROS trial in 2014, and probably also as a result of early reporting of the results at the annual meeting of the American Society of Clinical Oncology (ASCO) in 2013, it could be observed from the present registry data that ALND was being omitted in an increasing number of patients with one to three positive lymph nodes undergoing mastectomy. In 2013-2014, $22.4 \%$ of these patients were staged by SLNB alone, versus about $6 \%$ before publication.

Furthermore, the present study shows that elderly women with breast cancer (aged 80 years or above) underwent axillary staging less frequently than younger women, as has also been reported by others. ${ }^{36,37}$

The present study, using a large population-based registry, has demonstrated the radical transformation in management of the axilla since the introduction of SLNB and following the publication of recent studies. 


\section{References}

1. van de Vijver MJ, He YD, van't Veer LJ, Dai H, Hart AA, Voskuil DW, et al. A gene-expression signature as a predictor of survival in breast cancer. N Engl J Med. 2002;347:1999-2009.

2. Goldhirsch A, Glick JH, Gelber RD, Senn HJ. Meeting highlights: International Consensus Panel on the Treatment of Primary Breast Cancer. J Natl Cancer Inst. 1998;90:1601-8.

3. Liljegren G, Holmberg L. Arm morbidity after sector resection and axillary dissection with or without postoperative radiotherapy in breast cancer stage I. Results from a randomised trial. Uppsala-Orebro Breast Cancer Study Group. Eur J Cancer. 1997;33:193-9.

4. Ivens D, Hoe AL, Podd TJ, Hamilton CR, Taylor I, Royle GT. Assessment of morbidity from complete axillary dissection. Br J Cancer. 1992;66:136-8.

5. Noguchi M, Miwa K, Michigishi T, Yokoyama K, Nishijima H, Takanaka T, et al. The Role of Axillary Lymph Node Dissection in Breast Cancer Management. Breast Cancer. 1997;4:143-53.

6. Krag DN, Weaver DL, Alex JC, Fairbank JT. Surgical resection and radiolocalization of the sentinel lymph node in breast cancer using a gamma probe. Surg Oncol. 1993;2:335-9; discussion 40.

7. Ververs JM, Roumen RM, Vingerhoets AJ, Vreugdenhil G, Coebergh JW, Crommelin MA, et al. Risk, severity and predictors of physical and psychological morbidity after axillary lymph node dissection for breast cancer. Eur J Cancer. 2001;37:991-9.

8. Schijven $M$, Rutten $H$, Roumen R. Implementation of the sentinel node biopsy: a survey among surgeons in the Netherlands. Eur J Surg Oncol. 2000;26:431-2.

9. Schijven MP, Vingerhoets AJ, Rutten HJ, Nieuwenhuijzen GA, Roumen RM, van Bussel ME, et al. Comparison of morbidity between axillary lymph node dissection and sentinel node biopsy. Eur J Surg. 2003;29:341-50..

10. Lyman GH, Giuliano AE, Somerfield MR, Benson AB, 3rd, Bodurka DC, Burstein HJ, et al. American Society of Clinical Oncology guideline recommendations for sentinel lymph node biopsy in early-stage breast cancer. J Clin Oncol. 2005;23:7703-20.

11. Edge SB, Niland JC, Bookman MA, Theriault RL, Ottesen R, Lepisto E, et al. Emergence of sentinel node biopsy in breast cancer as standard-of-care in academic comprehensive cancer centers. J Natl Cancer Inst. 2003;95:1514-21.

12. Veronesi U, Paganelli G, Viale G, Luini A, Zurrida S, Galimberti V, et al. A randomized comparison of sentinel-node biopsy with routine axillary dissection in breast cancer. N Engl J Med. 2003;349:546-53.

13. Posther KE, McCall LM, Blumencranz PW, Burak WE, Jr., Beitsch PD, Hansen NM, et al. Sentinel node skills verification and surgeon performance: data from a multicenter clinical trial for early-stage breast cancer. Ann Surg. 2005;242:593-9.

14. Giuliano AE, Hunt KK, Ballman KV, Beitsch PD, Whitworth PW, Blumencranz PW, et al. Axillary dissection vs no axillary dissection in women with invasive breast cancer and sentinel node metastasis: a randomized clinical trial. JAMA. 2011;305:569-75.

15. Giuliano AE, McCall L, Beitsch P, Whitworth PW, Blumencranz P, Leitch AM, et al. Locoregional recurrence after sentinel lymph node dissection with or without axillary dissection in patients with sentinel lymph node metastases: the American College of Surgeons Oncology Group Z0011 randomized trial. Ann Surg. 2010;252:426-32.

16. Donker M, van Tienhoven G, Straver ME, Meijnen P, van de Velde CJ, Mansel RE, et al. Radiotherapy or surgery of the axilla after a positive sentinel node in breast cancer (EORTC 10981-22023 AMAROS): a randomised, multicentre, open-label, phase 3 non-inferiority trial. Lancet Oncol. 2014;15:1303-10.

17. Hermanek P SL, eds. UICC TNM classification of malignant tumors. Springer-Verlag, Berlin, pp 94991987.

18. Roumen RM, Pijpers HJ, Thunnissen FB, Ruers TJ. [Summary of the guideline 'Sentinel node biopsy in breast cancer.' Dutch Work Group 'Sentinel Node Biopsy for Breast Cancer']. Ned Tijdschr Geneeskd. 2000;144:1864-7.

19. Ho VK, van der Heiden-van der Loo M, Rutgers EJ, van Diest PJ, Hobbelink MG, Tjan-Heijnen VC, et al. Implementation of sentinel node biopsy in breast cancer patients in the Netherlands. Eur J Cancer. 2008;44:683-91. 
20. Tvedskov TF, Jensen MB, Balslev E, Ejlertsen B, Kroman N. Stage migration after introduction of sentinel lymph node dissection in breast cancer treatment in Denmark: a nationwide study. Eur J Cancer. 2011;47:872-8.

21. Maaskant AJ, van de Poll-Franse LV, Voogd AC, Coebergh JW, Tutein Nolthenius-Puylaert MC, Nieuwenhuijzen GA. Stage migration due to introduction of the sentinel node procedure: a population-based study. Breast Cancer Res Treat. 2009;113:173-9.

22. Gatzemeier W, Mann GB. Which sentinel lymph-node (SLN) positive breast cancer patient needs an axillary lymph-node dissection (ALND)--ACOSOG Z0011 results and beyond. Breast. 2013;22:211-6.

23. Maaskant-Braat AJ, Voogd AC, van de Poll-Franse LV, Coebergh JW, Nieuwenhuijzen GA. Axillary and systemic treatment of patients with breast cancer and micrometastatic disease or isolated tumor cells in the sentinel lymph node. Breast. 2012;21:524-8.

24. Singletary SE, Allred C, Ashley P, Bassett LW, Berry D, Bland KI, et al. Revision of the American Joint Committee on Cancer staging system for breast cancer. J Clin Oncol. 2002;20:3628-36.

25. Jörger M SH-J, Thürlimann B. St. Gallen 2009 recommendation on the treatment of early breast cancer: consensus and controversy. MEMO. 2009;2009;2:229-231.

26. Wright GP, Mater ME, Sobel HL, Knoll GM, Oostendorp LD, Melnik MK, et al. Measuring the impact of the American College of Surgeons Oncology Group Z0011 trial on breast cancer surgery in a community health system. Am J Surg. 2015;209:240-5.

27. Caudle AS, Hunt KK, Tucker SL, Hoffman K, Gainer SM, Lucci A, et al. American College of Surgeons Oncology Group (ACOSOG) Z0011: impact on surgeon practice patterns. Ann Surg Oncol. 2012;19:3144-51.

28. Caretta-Weyer H, Greenberg CG, Wilke LG, Weiss J, LoConte NK, Decker M, et al. Impact of the American College of Surgeons Oncology Group (ACOSOG) Z0011 trial on clinical management of the axilla in older breast cancer patients: a SEER-medicare analysis. Ann Surg Oncol. 2013;20:4145-52.

29. Dengel LT, Van Zee KJ, King TA, Stempel M, Cody HS, El-Tamer M, et al. Axillary dissection can be avoided in the majority of clinically node-negative patients undergoing breast-conserving therapy. Ann Surg Oncol. 2014;21:22-7.

30. Guth U, Myrick ME, Viehl CT, Schmid SM, Obermann EC, Weber WP. The post ACOSOG Z0011 era: does our new understanding of breast cancer really change clinical practice? Eur J Surg Oncol. 2012; 38:645-50.

31. Yi M, Kuerer HM, Mittendorf EA, Hwang RF, Caudle AS, Bedrosian I, et al. Impact of the american college of surgeons oncology group Z0011 criteria applied to a contemporary patient population. J Am Coll Surg. 2013;216:105-13.

32. Robinson KA, Pockaj BA, Wasif N, Kaufman K, Gray RJ. Have the American College of Surgeons Oncology Group 20011 trial results influenced the number of lymph nodes removed during sentinel lymph node dissection? Am J Surg. 2014;208:1060-4.

33. Gainer SM, Hunt KK, Beitsch P, Caudle AS, Mittendorf EA, Lucci A. Changing behavior in clinical practice in response to the ACOSOG Z0011 trial: a survey of the American Society of Breast Surgeons. Ann Surg Oncol. 2012;19:3152-8.

34. Massimino KP, Hessman CJ, Ellis MC, Naik AM, Vetto JT. Impact of American College of Surgeons Oncology Group Z0011 and National Surgical Adjuvant Breast and Bowel Project B-32 trial results on surgeon practice in the Pacific Northwest. Am J Surg. 2012;203:618-22.

35. Galimberti V, Cole BF, Zurrida S, Viale G, Luini A, Veronesi P, et al. Axillary dissection versus no axillary dissection in patients with sentinel-node micrometastases (IBCSG 23-01): a phase 3 randomised controlled trial. Lancet Oncol. 2013;14:297-305.

36. Wanebo HJ, Cole B, Chung M, Vezeridis M, Schepps B, Fulton J, et al. Is surgical management compromised in elderly patients with breast cancer? Ann Surg. 1997;225:579-86.

37. Mustacchi G, Cazzaniga ME, Pronzato P, De Matteis A, Di Costanzo F, Floriani I, et al. Breast cancer in elderly women: a different reality? Results from the NORA study. Ann Oncol. 2007;18:991-6. 



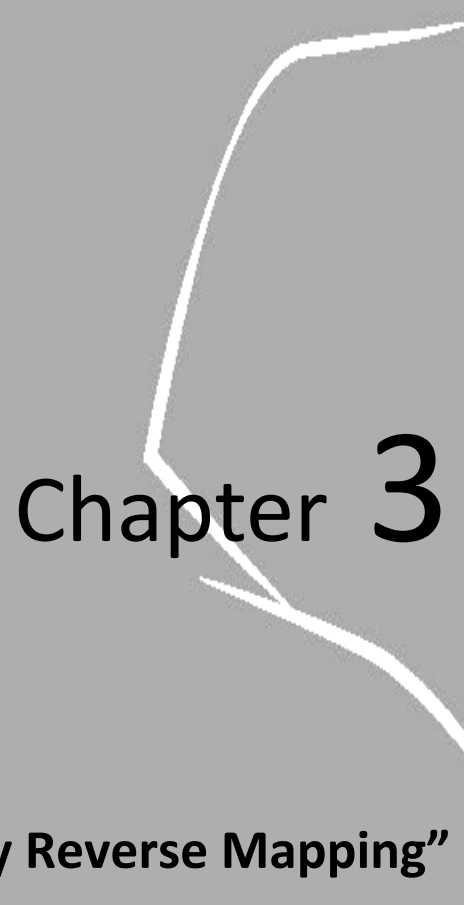
Is visualisation of lymphatics during axillary lymph

"Axillary Reverse Mapping" node dissection clinically relevant? Ned Tijdschr Geneesk 2013;157:A5646 


\section{Abstract}

A significant percentage of breast cancer patients who have undergone axillary lymph node dissection (ALND) suffer lymphedema afterwards. "Axillary reverse mapping" (ARM) is a technique which allows surgeons to visualise the lymphatic drainage system of the upper limb while performing ALND. If lymphedema after ALND is indeed caused by dissection of the axillary lymphatics or removing the attendant lymph nodes, preserving these structures might reduce the incidence of lymphedema. Patients with an indication for completion ALND, based on a positive sentinel lymph node may benefit from ARM, as may patients with an indication for primary ALND who were previously treated with neo-adjuvant chemotherapy (NAC). We will soon commence a multi-centre randomized clinical trial designed to determine whether preserving the axillary lymph nodes will reduce morbidity rates. 


\section{Which procedure to use?}

A few years ago, a technique was described which allowed intraoperative visualisation of the lymphatic drainage patterns from the arm: "axillary reverse mapping" (ARM). ARM is used to map the lymphatic drainage system of the upper limb, thus helping preserve lymphatics and lymph nodes during ALND. ${ }^{1}$ The ARM procedure starts with the injection of a marker which is preoperatively injected into the medial intermuscular groove of the ipsilateral upper arm, enabling surgeons to visualise the lymph nodes and lymphatics of the arm (Figure 3.1).

The ARM procedure is based on the anatomical position of the lymphatic drainage of the breast with respect to the upper limb. Both are located in the axilla, with the lymph nodes of the upper limb generally believed to be above the axillary vein, the upper boundary of the ALND.

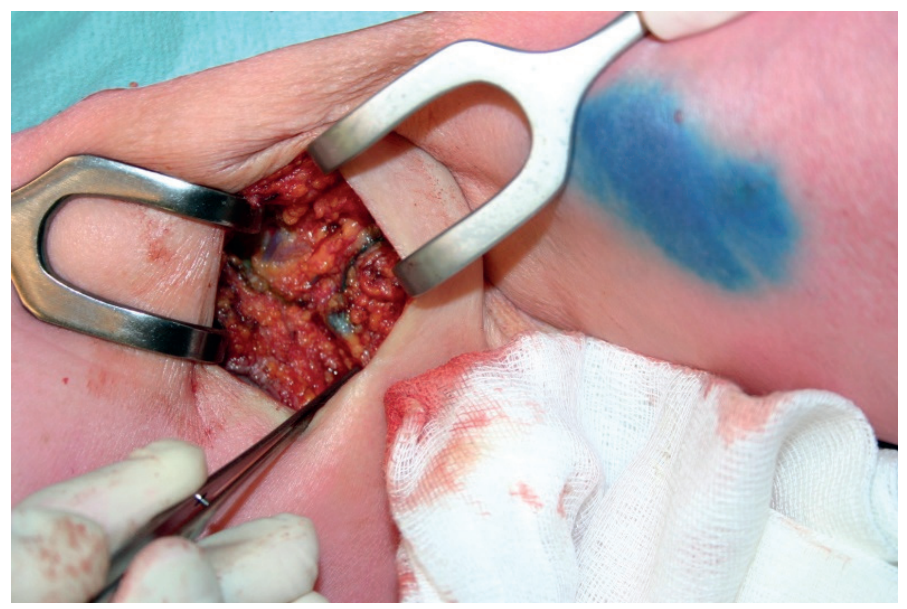

Figure 3.1 "Axillary reverse mapping" with blue dye causes the lymphatics and lymph nodes of the arm to turn blue.

However, some of the upper limb lymphatic drainage is actually drained below the level of the axillary vein, through the axillary nodal basin, which is generally completely removed during a standard ALND. These lymphatics and their corresponding lymph nodes (hereafter referred to as "ARM nodes") are situated in the upper, outer part of the axilla (Figure 3.2, zone D). During a standard ALND, these lymphatics and lymph nodes are generally removed. 
ARM can be performed using blue dye, fluorescence or radioisotopes. The latter two visualisation techniques have an advantage over blue dye in not leaving a blue, tattoolike stain on the patients skin. However, there is limited experience in using radioisotopes and fluorescence visualisation techniques. Blue dye, which is also used for sentinel lymph node procedures (SLNB) is an inexpensive tool and familiar with most (breast) surgeons. In addition, radioisotope and fluorescence imaging tends to require more expensive equipment.

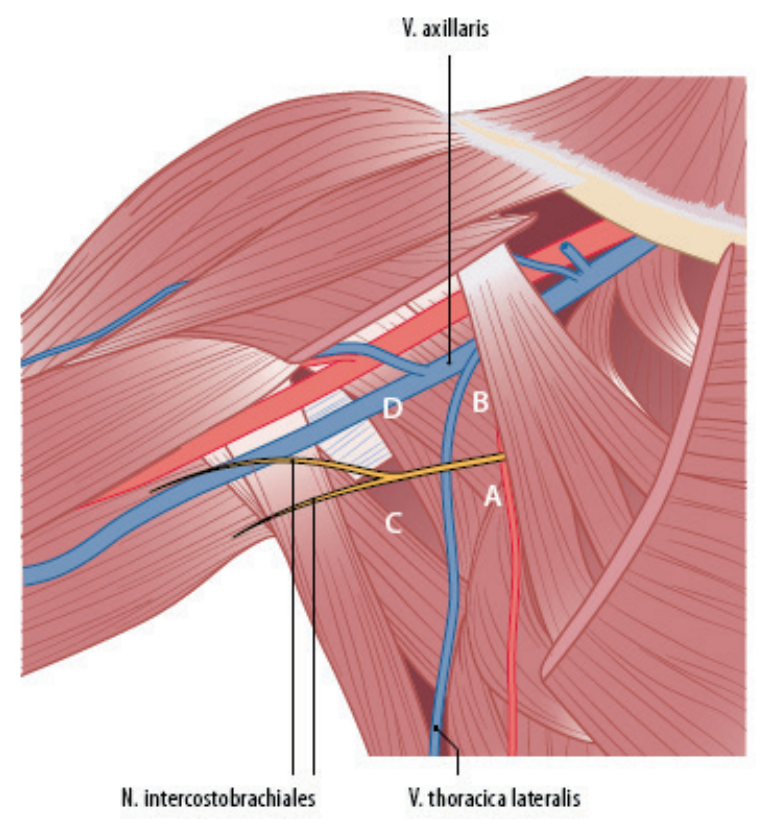

Figure 3.2 A schematic representation of the anatomy of the axilla. Where the lateral thoracic vein and the second intercostobrachial nerve intersect, the axilla is divided into four different axillary zones: $A, B, C$ and $D$. A and $B$ are considered the medial zone, while $C$ and $D$ are considered the lateral zone. Figure from Clough et al. ${ }^{4}$

\section{Why do we need a new technique?}

A significant percentage of breast cancer patients who have previously undergone ALND suffer some degree of lymphedema. Several smaller trials demonstrate this up to $70 \%$ (depending on the type of measurements used). Two more extensive randomised trials, NSABP B-32 and ALMANAC, show different results: $12-14 \%$. Even so, a reduction of morbidity in axillary surgery through improved surgical technique 
would be a welcome improvement. Using ARM while performing ALND may possibly reduce postoperative morbidity.

\section{For what indications?}

Patients with an indication for completion ALND (based on a positive SLN) may benefit from ARM, as this will help preserve the lymphatic draining system of the upper limb. In addition, patients with an indication for primary ALND and treated with neoadjuvant chemotherapy (NAC) may also be eligible for this procedure.

\section{What kind of problems does this technique solve?}

ALND's which preserve lymphatic drainage patterns (i.e. the lymphatics and the lymph nodes) may result in reduced postoperative morbidity, e.g. lymphedema.

\section{What do we know about the efficacy of the technique?}

A feasibility study carried out at our hospital $(n=93)$ demonstrated that ARM using blue dye can be used quite easily while performing an ALND to visualise ARM nodes situated in the axillary fat below the axillary vein (90.3\%). However, the study, which was designed to examine the blue dye visualisation percentage and the number of metastases in the ARM nodes, also resulted in a few other interesting findings. In patients who underwent completion ALND (after a positive SLN, $n=43$ ), the ARM nodes were always found to be tumour free. In this respect, these patients differed from patients who underwent primary ALND (because of a cytological confirmed axillary lymph nodes metastasis, $n=50$ ); $22 \%$ of whom were found to have metastases in the ARM nodes. Moreover, additional analysis of the latter group demonstrated a clear difference in the percentage of ARM nodes metastases in patients who had received NAC prior to the dissection $(n=38)$ compared to patients who received adjuvant chemotherapy $(n=12)$, respectively 15.8 and $41.7 \%{ }^{2}$ However, this difference was not believed to be significant, due to the relatively small group of patients included in the study $(n=50)$, which rendered a sub-group analysis hard to perform.

More recently, a small study has been published which shows that preserving the lymph drainage system of the upper limb really does reduce postoperative morbidity. ${ }^{3}$ 


\section{Is this technique easy to learn?}

In our series, over $90 \%$ of the patients involved were successfully operated using ARM. Surgeons who are experienced at performing ALND and SLNB are generally able to master the technique within a few procedures.

\section{Future expectations}

Axillary treatment in breast cancer patients has been under debate the last few years. A recently published ${ }^{5}$ American multi-centre study, ACOSOG Z0011 study, randomised breast cancer patients with one to three positive sentinel nodes to either completion ALND or no further axillary treatment. At a median follow-up of 6.3 years, there was no significant difference in overall and disease-free survival rates between the two groups. The locoregional recurrence percentage was $0.9 \%$ in women who did not undergo ALND. On the other hand, $27 \%$ of patients who did undergo ALND were found to have SLN metastases, which may potentially result in cancer recurrence in the group of patients who were received conservative treatment and did not undergo completion ALND.

The recently reviewed 'Breast Cancer 2.0' guideline (which can be found on www.oncoline.nl) has been significantly revised. For instance, it no longer provides an unequivocal recommendation on how to treat patients with SLN metastases. Treatment options now include completion ALND, axillary radiotherapy and no further axillary treatment.

Even so, it will probably take a while before the axilla will be left untreated following a positive SLN. This is not just due to the inclusion protocol of the ACOSOG Z0011 study, which only included breast cancer patients who underwent breast conserving therapy, in $96 \%$ of all cases, adjuvant systemic therapy, but also due to other aspects of the trial, such as its statistical limitations, the low percentage of secondary axillary nodes metastases (27\%), the strikingly high percentage of micrometastases, and the low number of patients per participating centre. ${ }^{6}$ Nevertheless, nowadays there are several hospitals in the Netherlands which no longer perform completion ALND on SLN positive patients.

Because of this 'unclear-axillary-era', and the fact that there will always be patients who will need ALND it is worth investigating the clinical relevance of axillary reverse mapping and preserving the ARM nodes, for node-positive patients who have been advised to undergo ALND and who have previously received breast-conserving surgery 
or breast amputation, and possibly also for patients with an indication for primary ALND after receiving NAC.

\section{Randomised study}

Our hospital will soon start a randomised multi-centre study in which SLN positive patients with an indication for completion ALND will be randomised either to standard-ALND or to an ARM-ALND, which will preserve the lymphatics and lymph nodes of the upper extremity. Breast cancer-related lymphedema, paraesthesia, numbness, pain and reduced arm and shoulder mobility will be the primary outcome measures of the study. Quality of life and cancer recurrence in the axilla will be secondary outcome measures. Due to the need for a sub-group analysis of clinically node positive patients, patients with an indication for primary ALND will be enrolled in a separate registration study. As part of this study, they will undergo ARM, but their lymph nodes and lymphatics will be removed on a separate occasion (which is to say that they will not be preserved). The aim of the separate registration study is to determine the extent to which these patients, who often receive NAC, may be eligible for ALND using ARM in future.

\section{Where in the Netherlands?}

We have recently been authorised by the Medical Ethics Review Board to commence investigating the efficacy of ARM in the Amphia Hospital in Breda, Catharina Hospital in Eindhoven, Haga Hospital in The Hague and Gelderse Vallei Hospital in Ede. We expect to be able to commence the study in these hospitals in the course of 2013.

Contributions to the New Techniques section outline potential new techniques in the field of medicine which are so new that they have not yet been proven valid, but come with so many interesting facts that our readers are likely to find the information contained in the story quite useful. Whether or not the techniques described in the articles will end up becoming standard procedure following further trials remains to be seen. 


\section{References}

1. Noguchi M. Axillary reverse mapping for breast cancer. Breast Cancer Res Treat. 2010;119:529-35.

2. Gobardhan PD, Wijsman JH, van Dalen T, Klompenhouwer EG, van der Schelling GP, Los J, et al. ARM: axillary reverse mapping - the need for selection of patients. Eur J Surg Oncol. 2012;38:657-61.

3. Boneti C, Badgwell B, Robertson Y, Korourian S, Adkins L, Klimberg V. Axillary reverse mapping (ARM): initial results of phase II trial in preventing lymphedema after lymphadenectomy. Minerva Ginecol. 2012;64:421-30.

4. Clough KB, Nasr R, Nos C, Vieira M, Inguenault C, Poulet B. New anatomical classification of the axilla with implications for sentinel node biopsy. Br J Surg. 2010;97:1659-65.

5. Giuliano AE, Hunt KK, Ballman KV, Beitsch PD, Whitworth PW, Blumencranz PW, et al. Axillary dissection vs no axillary dissection in women with invasive breast cancer and sentinel node metastasis: a randomized clinical trial. JAMA. 2011;305:569-75.

6. Kuhn T, Poortmans PhMP. Zoo11 - Can We Really Abolish Axillary Dissection? Breast Care 2011;6:154-7. 2011.

7. Gobardhan PD, Klompenhouwer EG, Beek MA, Voogd AC, Luiten EJ. [Axillary reverse mapping. preserving nodes during an axillary lymph node dissection]. Ned Tijdschr Geneeskd. 2013;157:A5646. 


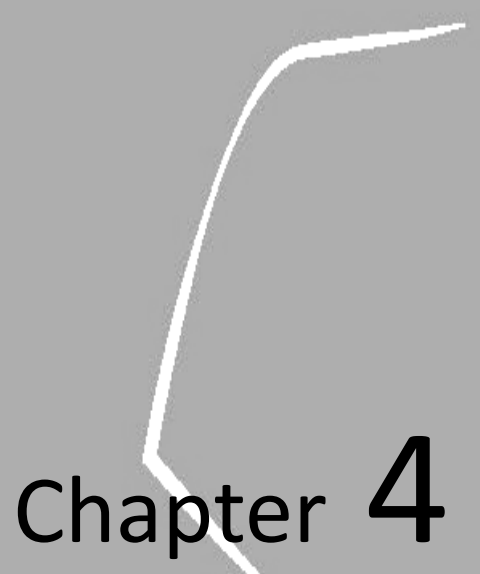

Axillary reverse mapping in axillary surgery for breast cancer - an update of the

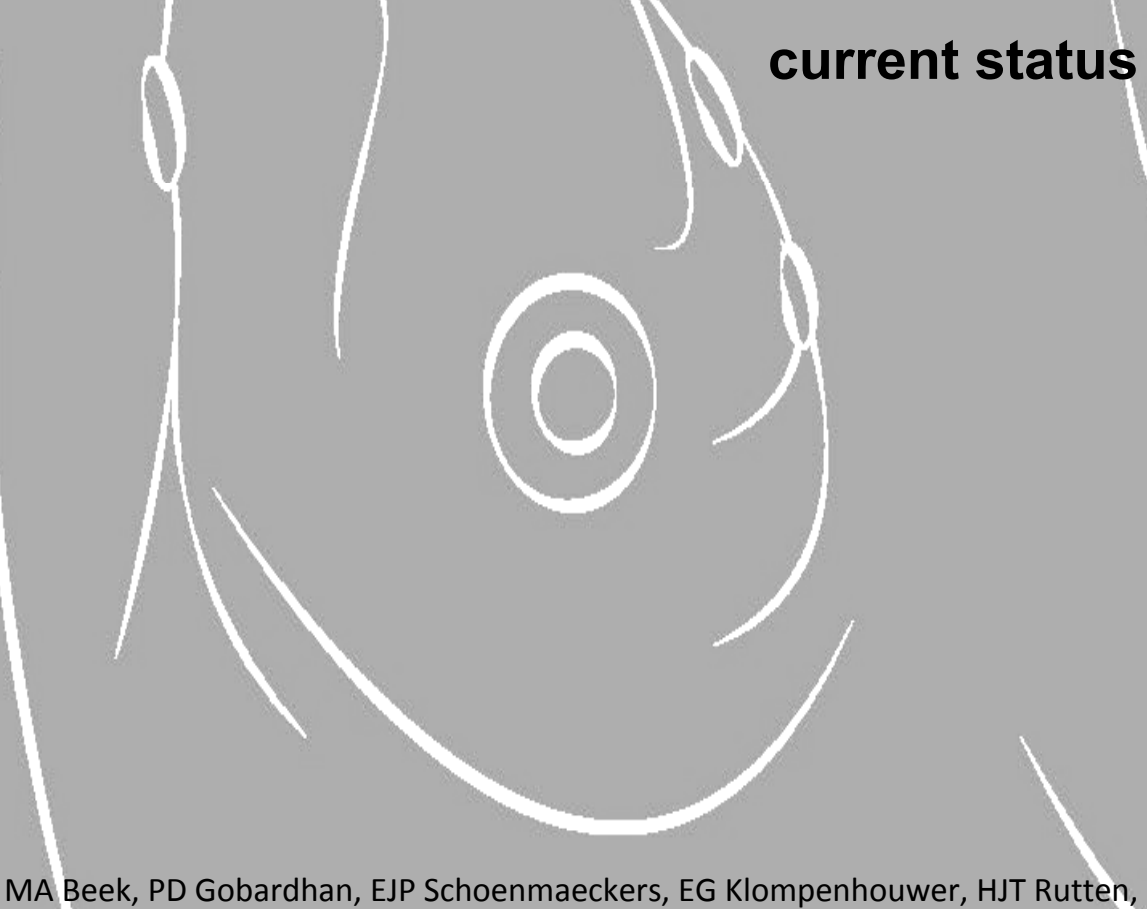
AC Voogd, EJT Luiten Breast Cancer Res Treat 2016;158:421-432 


\section{Abstract}

\section{Background}

Axillary reverse mapping (ARM) is a technique by which the lymphatic drainage of the upper extremity that traverses the axillary region can be differentiated from the lymphatic drainage of the breast during axillary lymph node dissection (ALND). Adding this procedure to ALND may reduce upper extremity lymphedema by preserving upper extremity drainage. This review of the current literature on the ARM procedure discusses the feasibility, safety and relevance of this technique.

\section{Method}

A PubMed literature search was performed until 12 August 2015. A total of 31 studies were included in this review.

\section{Results}

The studies indicated that the ARM procedure adequately identifies the upper extremity lymph nodes and lymphatics in the axillary basin using blue dye or fluorescence. Preservation of ARM lymph nodes and corresponding lymphatics was proven to be oncologically safe in clinically node-negative breast cancer patients with metastatic lymph node involvement in the sentinel lymph node (SLN) who are advised to undergo a completion ALND.

\section{Conclusion}

The ARM procedure is technically feasible with a high visualisation rate using blue dye or fluorescence. ALND combined with ARM can be regarded as a promising surgical refinement in order to reduce the incidence of upper extremity lymphedema in selected groups of patients. 


\section{Introduction}

Before the introduction of the sentinel lymph node biopsy (SLNB) in the early 1990s, axillary lymph node dissection (ALND) was the standard care in the treatment of breast cancer patients. Although the SLNB introduced a minimally invasive procedure for breast cancer staging, a substantial proportion of breast cancer patients are still scheduled for primary or completion ALND for tumour staging and optimising locoregional control. ${ }^{1,2}$

Although ALND is still the gold standard in primary clinically lymph node-positive patients, the procedure is associated with substantial morbidity consisting of upper extremity lymphedema (7-77\%), ${ }^{3-10}$ arm/shoulder dysfunction, paresthesia and discomfort. $^{11}$

In 2007, the first reports ${ }^{12,13}$ on axillary reverse mapping (ARM) - a procedure that enables discrimination of the upper extremity and breast lymphatic drainage in the axillary region - were published. The fundamental concept of the ARM procedure is based on the assumption that the upper extremity and the breast each has their own discrete pathways of lymphatic drainage through the axilla. Interconnections between these pathways have been reported. ${ }^{14}$ This concept was tested and validated in a mouse model in which two different drainage pathways were demonstrated by twocolour spectral fluorescence lymphangiography. ${ }^{15}$ If upper extremity lymphedema is caused by the disruption of corresponding axillary lymphatics and/or removal of the upper extremity (ARM) lymph nodes, then the ability to identify and preserve them could possibly prevent upper extremity lymphedema during axillary surgery.

Ever since, the concept of ARM has drawn more attention and several studies have reported on the clinical utility of this possible improvement in the technique of ALND in an effort to reduce morbidity while preserving oncologic safety.

In this update regarding ARM in breast cancer patients, we present an overview of the current literature with regard to the feasibility of the ARM technique, oncologic safety and the reduction of lymphedema by preservation of the ARM lymph nodes and corresponding upper extremity lymphatics during ALND.

\section{Method}

\section{Study selection}

A PubMed literature search was performed until 12 August 2015. 'Axillary reverse mapping' was used as the search term, which was restricted to the title, abstract and keywords. Only studies published in English were considered.

All cross-references were screened for potentially relevant studies that had not been identified by the initial literature search. Only studies including at least 10 patients 
undergoing the ARM procedure in breast cancer patients were included. Results of the literature search are summarised in Figure 4.1. Baseline characteristics of the studies included are shown in Table 4.1.

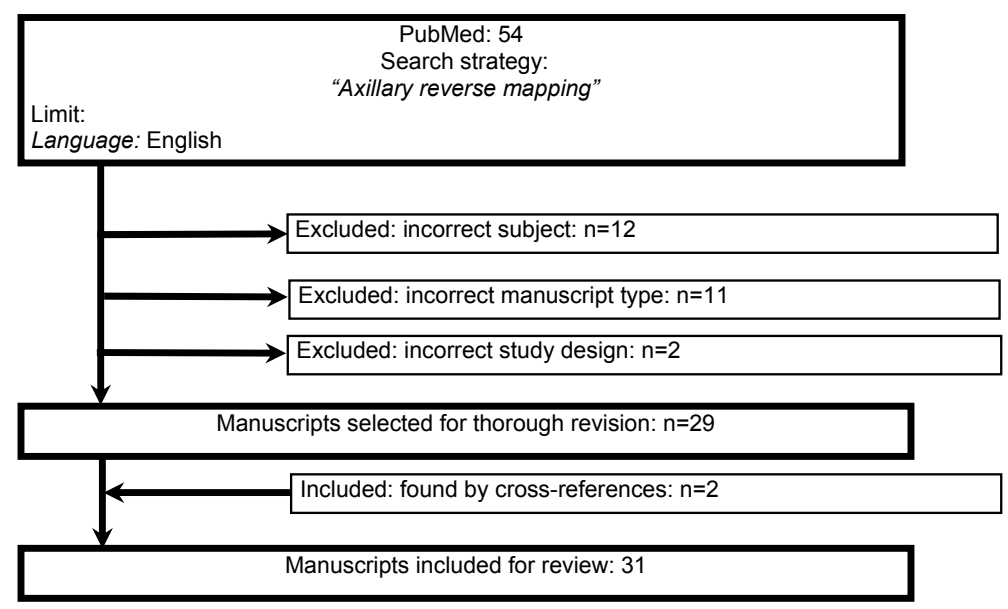

Figure 4.1 Results of the literature search (12 August 2015).

\section{Data extraction}

The variables that were analysed were as follows: number of patients treated; number of ARM procedures; number of ARM lymph nodes and/or ARM lymphatics identified in the ALND field; number of ARM lymph nodes and/or ARM lymphatics identified in the SLNB field; number of ARM lymph nodes with metastatic involvement; number of converged sentinel lymph node (SLN)-ARM lymph nodes; and the incidence of upper extremity lymphedema.

\section{Technique and feasibility of axillary upper extremity lymph node visualisation by the ARM procedure}

Three visualisation techniques have been described: radioisotopes, fluorescence dye and blue dye. Blue dye is most often used (Table 4.1). 


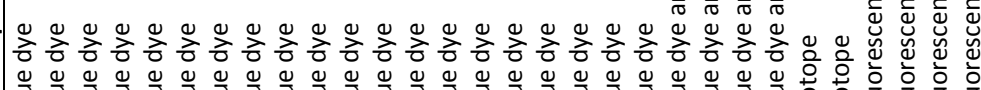

응 응

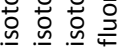

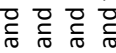

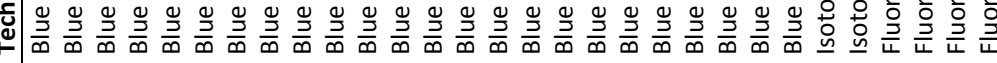

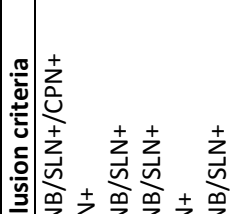

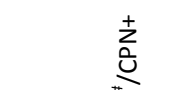

$\sum_{\substack{0 \\ \vdots}}^{+}$

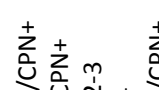

$\sum_{\substack{0 \\ 0}}^{+}$

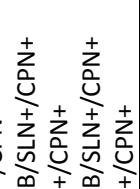

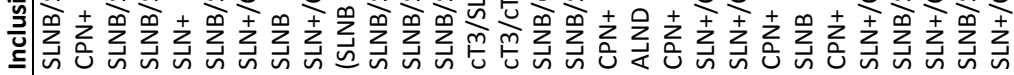

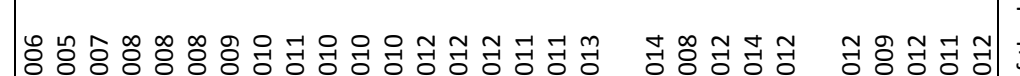

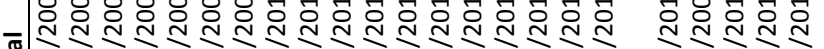

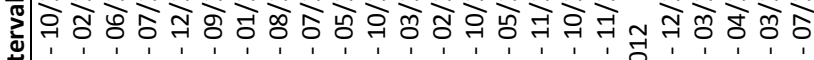

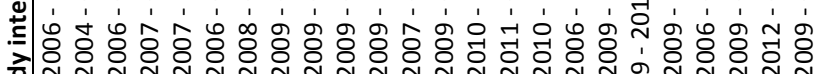

on

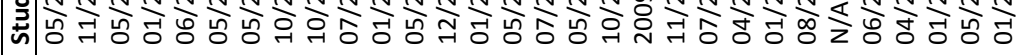

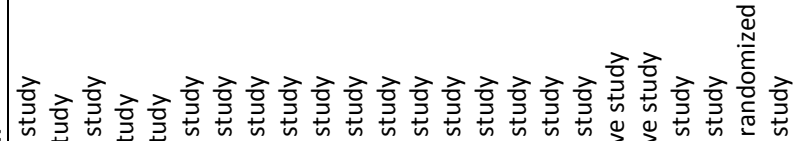

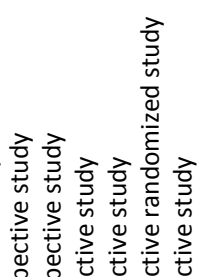

సิ

$1,1,1$

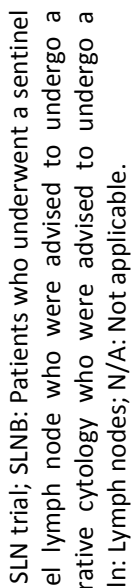

엉 实

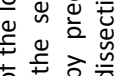

$t . \subseteq \simeq \frac{8}{0}$

주웡

들 을

之造

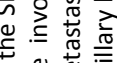

फั0 웜

节

है

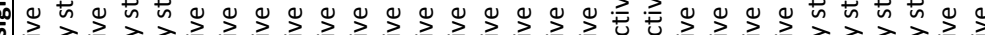

贾

늘

응

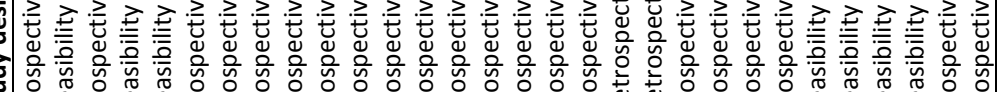

:

齐

눈흔

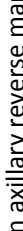

$\frac{0}{\frac{n}{0}}$

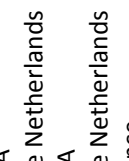

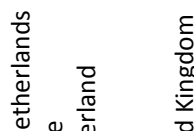

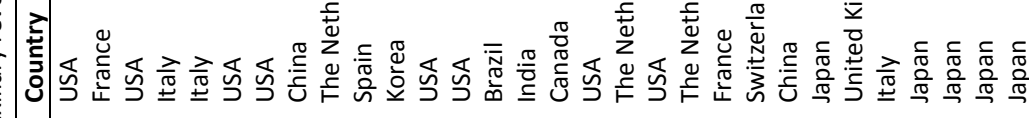

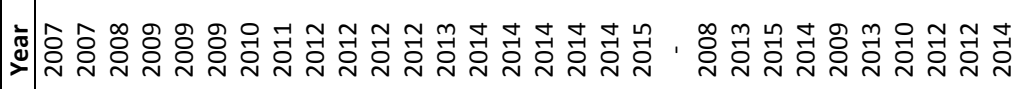




\section{Visualisation techniques}

\section{Blue dye}

The initial studies on ARM in 2007 used a blue dye to map axillary upper extremity lymph nodes and lymphatics. ${ }^{13,12}$ In accordance with the initial reports on ARM in 2007 by Nos and Thompson, respectively, blue dye has been used most often to map the axillary upper extremity lymph drainage (Table 4.1).

Visualisation rates in the axilla of ARM lymph nodes during ALND ranged from $39 \%$ to $90 \%$ and of ARM lymphatics from $47 \%$ to $86 \%$ (Table 4.2 ). The visualisation rates during SLNB of ARM lymphatics ranged from $20 \%$ to $47 \%$ (Table 4.2 ). Notably, three studies reported no data on visualisation rates since they only included patients in whom a successful ARM procedure was achieved. ${ }^{16,17}$ Furthermore, another study also reported no data on visualisation rates since it was done by a retrospective questionnaire survey. ${ }^{18}$

The wide range of visualisation rates (20-90\%) may possibly be explained by several factors. First, the possibility of encountering upper extremity lymphatics or corresponding nodes is dependent on the extent of axillary surgery. In patients who underwent SLNB only, visualisation rates are therefore lower as compared to patients who underwent an ALND. Casabona et al. ${ }^{19}$ reported a visualisation rate of $89 \%$ of ARM lymphatics in the ALND field and $38 \%$ of ARM lymphatics in the SLNB field. Two other recent studies reported the same difference in visualisation rates between the SLNB and ALND fields ${ }^{20,21}$ (Table 4.2). These differences in visualisation rates between SLNB and ALND fields can be explained by taking into account the anatomical locations of the ARM lymph nodes and the SLN. Using the anatomic classification of Clough et al., ${ }^{22}$ ARM lymph nodes are most commonly found in the upper outer quadrant of the axilla just caudal of the axillary vein (Figure 4.2D) and lateral from the ascending lateral thoracic vein that ends in the axillary vein, while the SLN is most frequently located in the lower basin of the axilla (Figure 4.2A and C). ${ }^{23,24,19,25-28}$

Secondly, the definition of a successful ARM procedure differs between the reported studies. Some studies reported visualisation rates of only ARM lymphatics, while other studies reported visualisation rates of only ARM lymph nodes and, finally, some studies reported both. Bedrosian et al. reported visualisation rates of ARM lymph nodes in $50 \%$ and ARM lymphatics in $70 \% .{ }^{23}$ These differences with regard to visualisation rates were also reported by Ponzone et al. ${ }^{27}$ (55\% ARM lymph nodes vs. $69 \%$ ARM lymphatics) and Thompson et al. ${ }^{12}$ (39\% ARM lymph nodes vs. 61\% ARM lymphatics; Table 4.2). 


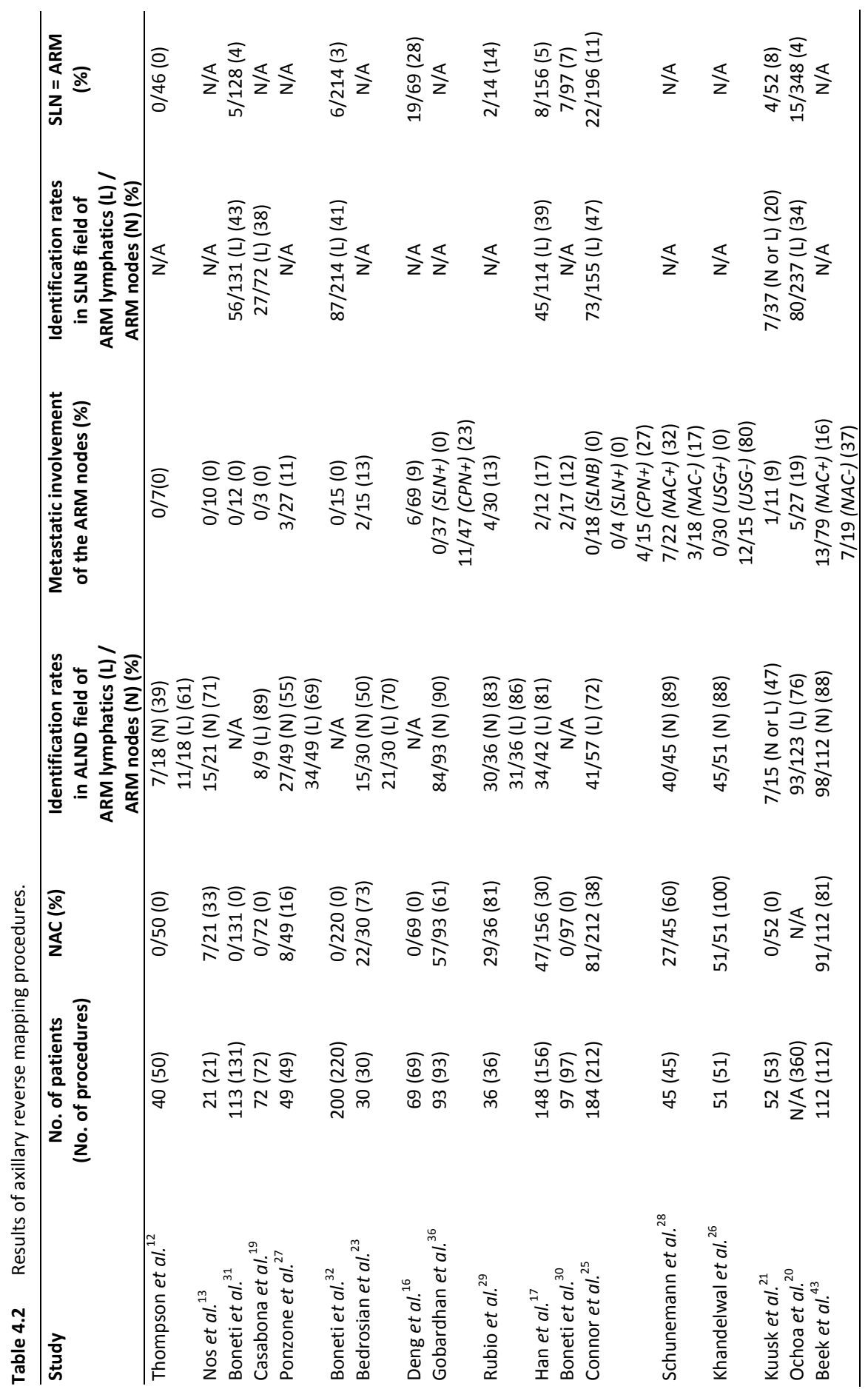




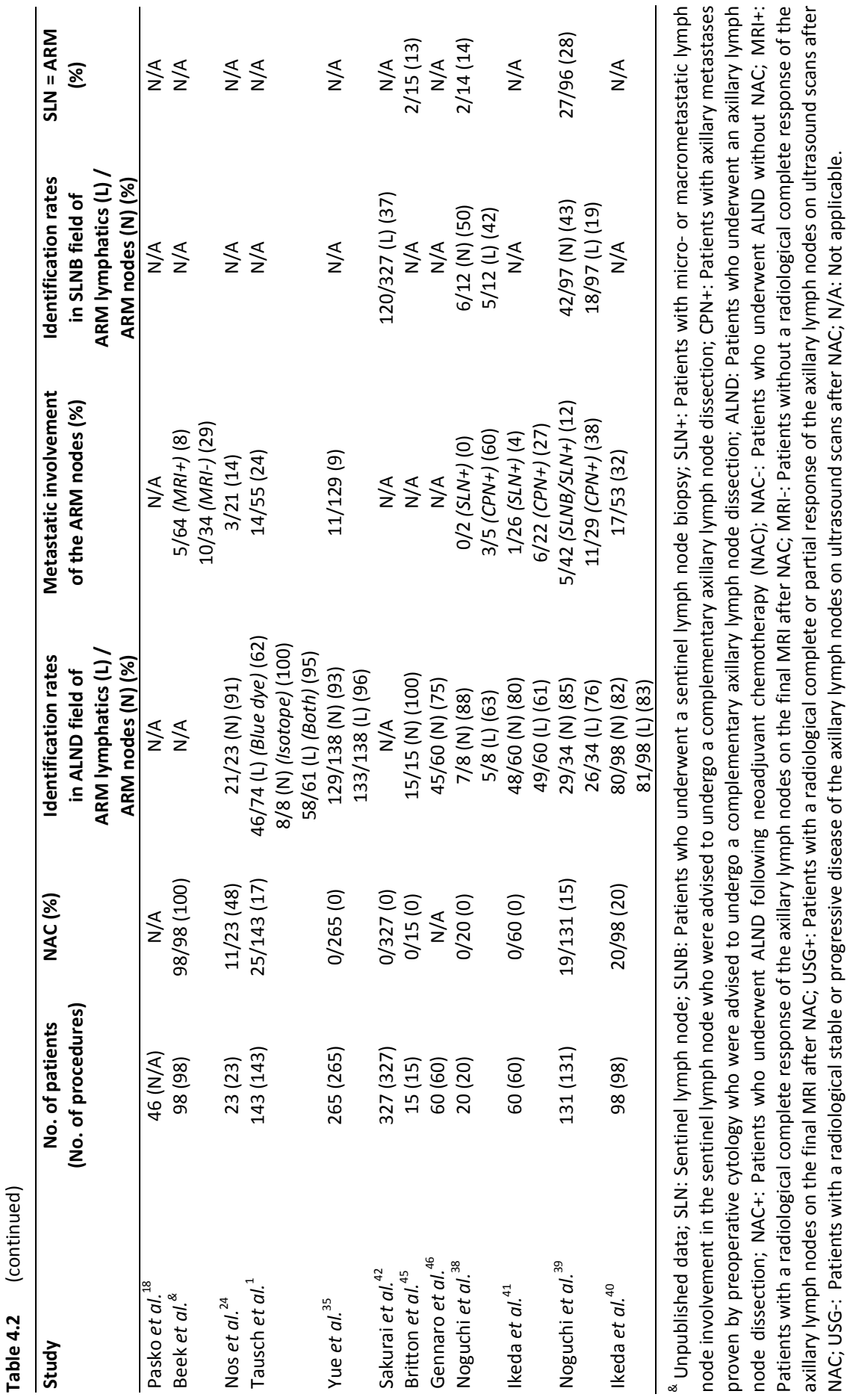




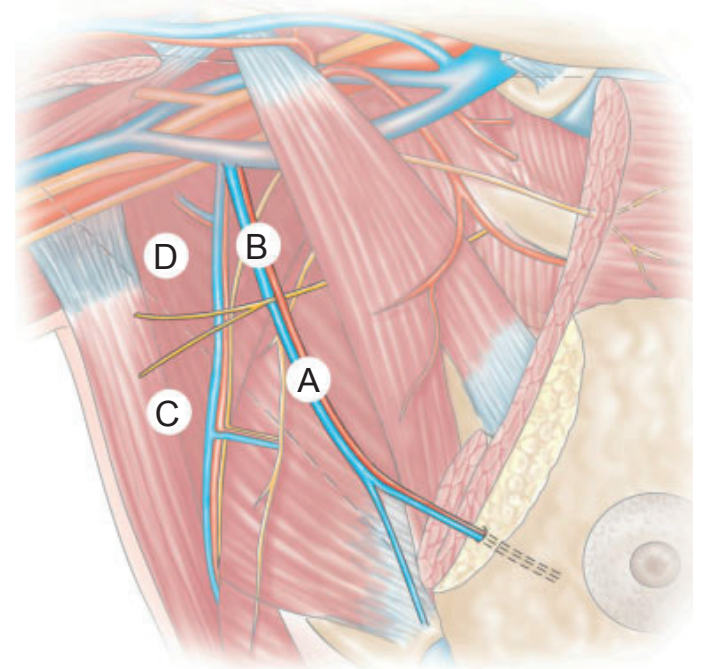

Figure 4.2 Anatomic classification of the axilla (Clough et al. ${ }^{22}$ ).

Technical aspects of the ARM procedure are a possible third factor that might explain the difference in visualisation rates. The time interval between injection of the blue dye and the start of the operation and insufficient intraoperative massage time of the injection spot following injection of blue dye could possibly influence the success rate of the procedure. ${ }^{1,13,29}$ Besides, different volumes of blue dye were used, ranging from 1 to $5 \mathrm{~mL}$. Six studies used 2-5 mL blue dye ${ }^{12,26,30-33}$ compared to the $1 \mathrm{~mL}$ of blue dye that was used in two other studies. ${ }^{27,21}$ The visualisation rates of the studies using 2 $5 \mathrm{~mL}$ blue dye were much higher than the visualisation rates reported by Ponzone et al. and Kuusk et al. who used only $1 \mathrm{~mL}$ (Table 4.2). This suggests that a volume of at least $2 \mathrm{~mL}$ is most appropriate in performing ARM, as was also recommended by Klimberg. ${ }^{34}$

Finally, the experience of the surgeon in performing the ARM procedure may have an impact on the success rate. It has been suggested that the learning curve is similar to the SLNB procedure and an increase in the detection rates of ARM lymph nodes using blue dye is noted with increasing surgical exposure. ${ }^{13,32,35,36}$

\section{Complications and technical considerations}

Complications due to blue dye are uncommon; reports of anaphylactic reactions, ${ }^{1}$ local skin reactions ${ }^{12}$ and a transient drop in saturation ${ }^{26,29}$ are rare. Subcutaneous 
injection of blue dye leaves a 'blue tattoo' at the injection site that will disappear after a period of several weeks to more than a year. ${ }^{13,19,21,26}$

If the ARM procedure is carried out during a standard dual tracer (blue dye and Tc-99) SLNB, simultaneous use of the blue dye for ARM makes it almost impossible to adequately discriminate the ARM lymph nodes and lymphatics from the SLN. In these situations, the use of fluorescence dye or radioisotopes may be considered.

\section{Radioisotopes (Tc-99)}

The first study concerning the ARM procedure using a radioisotope was published in 2008 by Nos et al. ${ }^{24}$ (Table 4.1). In this study, a dual tracer technique using blue dye to visualise ARM lymphatics and Tc-99 to identify ARM lymph nodes resulted in visualisation rates of in $91 \%$ of all patients. Similar results with Tc-99 (with or without blue dye) have been reported by others (Table 4.2).

However, the ARM procedure using only Tc-99 cannot be carried out simultaneously with the dual tracer SLN technique in which Tc-99 is also used. Apart from the longer duration of the surgery, a major limitation of the use of Tc-99 is the impossibility of mapping the ARM lymphatics, which also have to be preserved in order to perform a complete ARM procedure. ${ }^{1,24,35}$

\section{Fluorescent dye}

Up until now, only five studies have reported on the use of a fluorescent dye (indocyanine green). ${ }^{37}$ In a pilot study reported by Noguchi et al. in 2010, visualisation rates of the ARM lymph nodes and the ARM lymphatics in the ALND field were $88 \%$ and $63 \%$, respectively (Table 4.2 ). In the same study, visualisation of the ARM lymph nodes and the ARM lymphatics during SLNB was only $43 \%$ and $19 \%$, respectively. In a subsequent larger study published in 2012, Noguchi et al. achieved an identification rate of $85 \%$ of ARM lymph nodes in the ALND field. ${ }^{38}$ However, lower identification rates were reported during SLNB due to the fact that ARM lymph nodes are anatomically located at a higher level in the axilla than the SLN similar when using a blue dye. ${ }^{39}$ An increase in the detection rate depending on surgical experience was reported by lkeda et al. using either fluorescent dye or blue dye. ${ }^{39}$

A major advantage of using indocyanine green compared to other techniques is the possibility of combining the ARM procedure with standard dual tracer SLNB. Furthermore, no systemic allergic reactions have been reported and the 'green tattoo' disappears somewhat quicker compared to the blue dye (within days up to a few months). ${ }^{37-41}$

In conclusion, three visualisation techniques have been used when performing ARM. If upper extremity lymphedema is caused by disruption of the ARM nodes and/or removal of the ARM lymph lymphatics, there will be no place for mapping the axilla 
using only Tc-99. Visualisation using blue dye might be preferred since it is easy to perform and inexpensive. However, when using the blue dye technique, a 'blue tattoo' will be seen on the patient's skin following subcutaneous injection. Visualisation using Tc-99 may have higher identification rates when compared to blue dye but does not allow visualisation of the ARM lymphatics, which is considered essential when lymphatic drainage of the upper extremity should be kept intact. Visualisation using fluorescence dyes results in high visualisation of both ARM lymph nodes and lymphatics of up to around $90 \%$. Studies on the former are scarce, however. Moreover, this technique requires the use of additional equipment in contrast to the use of blue dye, which is available in almost every hospital.

\section{Clinical utility of ARM in different stages of breast cancer: patient selection and oncologic safety}

The concept of the ARM procedure is based on the hypothesis that axillary lymph node draining of the upper extremity may play a minor role in the lymph node metastasis pathway of breast cancer. However, interconnections between these pathways do exist, thereby enabling metastatic breast cancer cells to invade ARM lymph nodes as well. Until now, no increased incidence of axillary recurrence has been reported in the literature when the ARM lymph nodes were preserved (Table 4.2). When reviewing the oncologic safety of the ARM procedure, there appears to be a significant variability in patient selection. Therefore, we have classified and divided the patients into three groups according to their axillary status. The first group (SLNB patients) consisted of patients with clinically node-negative breast cancer who underwent SLNB. The second group (SLN+ patients) consisted of patients with microor macrometastatic lymph node involvement in the SLN who were advised to undergo a completion ALND. The third group (CPN+ patients) consisted of patients who had a preoperative (proven by fine-needle aspiration cytology) diagnosis of lymph node metastasis and were scheduled for primary ALND.

\section{SLNB in cNo patients}

Twelve studies reported on the oncologic safety of SLNB patients (Table 4.1). None of them found metastatic involvement of the ARM lymph nodes in SLN-negative cases. A study by Deng et al. showed metastatic involvement of the ARM lymph node in 6 of 69 patients. They observed that in all these patients, the ARM lymph nodes were concordant with the breast SLN lymph nodes. ${ }^{16}$ This finding has also been confirmed by others; metastatic involvement of non-SLN-ARM lymph nodes was never seen and preserving these lymph nodes is oncologically safe. ${ }^{16,37,38}$ 


\section{Completion ALND in SLN+ patients}

Several studies reported no metastatic involvement in the ARM lymph nodes in patients with micro- or macrometastatic SLN involvement in whom a completion ALND was carried out, regardless of the presence of additional metastases in other lymph nodes in the ALND specimens (Table 4.2). Gobardhan et al. $^{33}$ found no metastatic involvement in $37 \mathrm{SLN}+$ patients, which has also been reported by Casabona et al. ${ }^{19}$ in three SLN+ patients and by Conner et al. ${ }^{25}$ in a subgroup of four patients. Noguchi et al. ${ }^{38}$ showed metastatic involvement of the ARM lymph nodes, all of which were concordant with the breast SLN, in 5 of 42 patients. The other studies that included SLN+ patients also included other groups of patients, which makes it difficult to draw conclusions on this subgroup (Table 4.2).

\section{Primary ALND in CPN+ patients}

In 2008, Nos et al. were the first to report on the metastatic involvement of ARM lymph nodes in clinically node-positive patients ${ }^{24}$ (Table 4.2), thereby casting doubt on the oncologic safety of preservation of ARM lymph nodes and corresponding lymphatics in this subgroup of patients. In this study of 21 patients with a successful identification of the ARM lymph nodes, three (14\%) were found to harbour metastatic involvement of the ARM lymph nodes. This finding was confirmed by others, with metastatic involvement of the ARM lymph nodes up to $60 \%$. Notably, higher rates of axillary ARM node metastatic involvement were mostly reported in smaller studies (Table 4.3). Some of these studies reported on a correlation between axillary stage and the number of positive ARM lymph nodes. ${ }^{35,39}$

Nowadays, all breast cancer patients with clinically node-positive disease undergo neoadjuvant systemic therapy (NAC) followed by breast surgery and ALND. NAC results in an axillary pathologic complete response ( $\mathrm{PCR}$ ) in one out of three patients, indicating the complete absence of axillary metastases after completion of NAC. A trend towards performing the SLN procedure or targeted axillary dissection is noted following NAC, although high false-negative rates of up to more than $20 \%$ have been reported. ${ }^{42}$ In the case of a positive SLN following NAC, the indication for additional axillary treatment is evident. Completion ALND remains the gold standard in these cases, although replacement by radiotherapy is noted even though there is no Level I clinical evidence so far. In patients undergoing direct breast reconstruction with tissue expander following breast amputation, radiotherapy should be avoided as much as possible to prevent complications and worse cosmetic outcome. 


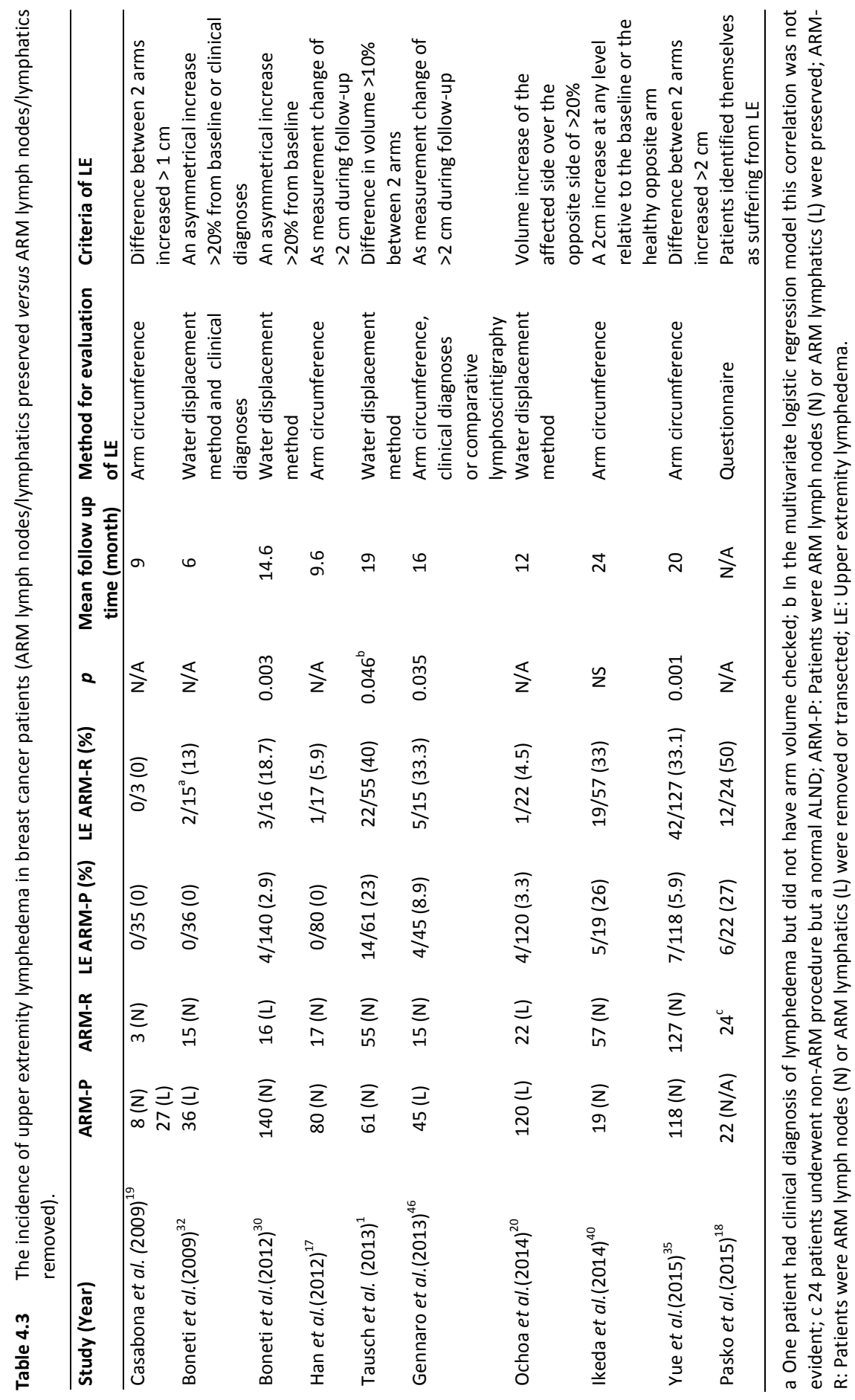


Beek et al. found a significant decrease in metastasis in the ARM lymph nodes for those in whom a primary ALND was preceded by NAC in CPN+ patients ${ }^{43}$ (Table 4.2). In a recent analysis, they found that a radiologically complete response of the axilla on standard breast MRI in CPN+ patients treated with a primary ALND following NAC was correlated with less metastatic involvement of ARM lymph nodes. ARM lymph nodes contained metastasis in 5 out of 57 patients with a radiologically complete response of the axilla, resulting in a negative predictive value of $91 \%$ (Table 4.2 ). ${ }^{44}$ This decrease following NAC was also reported by Khandelwal et al. using ultrasound of the axilla pre- and post-NAC. In this study, none of the ARM lymph nodes contained metastatic involvement in patients with partial or complete response following NAC. ${ }^{26}$

In conclusion, in both SLNB patients and SLN+ patients, it is oncologically safe to preserve the ARM lymph nodes provided that no convergence of the ARM lymph nodes and SLN is present. In CPN+ patients, some studies have reported metastatic involvement of the ARM lymph nodes, casting doubt over the oncological safety of preserving the ARM lymph nodes. However, in patients with a clinically and radiologically complete response following NAC, reports of the absence of metastasis in the ARM lymph nodes may open the way to preserving them in this selected group of patients. A further selection of $\mathrm{CPN}+$ patient groups might benefit from this technique, and should be the subject of future research.

\section{Clinical impact of ARM with regard to the reduction of upper extremity lymphedema}

Upper extremity lymphedema is one of the most serious postoperative complications in patients during axillary surgery. Although the development of upper extremity lymphedema is related to the removal of axillary lymph nodes, other factors do play a role, e.g. radiotherapy of the axilla or obesity. ${ }^{45}$ In patients who underwent SLNB, occurrence of upper extremity lymphedema is up to $13 \%$. In patients who underwent standard ALND, the incidence was significantly higher, ranging from $7 \%$ to $77 \% .{ }^{3-10}$ In order to evaluate the clinical utility of ARM with preservation of ARM lymph nodes and corresponding lymphatics with regard to the reduction of upper extremity lymphedema, SLNB patients and ALND patients have to be analysed separately.

In patients who underwent SLNB only, the incidence of upper extremity lymphedema can be explained by the concordance of SLN and ARM lymph nodes, both of which drain into the same lymph node. ${ }^{46}$ Several studies have reported crossover rates of up to $28 \%$ between ARM lymph nodes and SLN (Table 4.2).

If the ARM lymph nodes are concordant with the SLN draining the breast, there is no possibility of preserving them, which may serve as a possible explanation for the incidence of upper extremity lymphedema in patients after SLNB. Since ARM lymph 
nodes may be situated in the immediate surroundings of the SLN, extensive dissection may cause accidental damage to these lymph nodes and lymphatics, thereby compromising upper extremity lymphatic drainage.

Ten studies reported on the incidence of upper extremity lymphedema in patients who underwent ALND with or without preserving the ARM lymph nodes and/or lymphatics (Table 4.3). The incidence of upper extremity lymphedema was evaluated by different measurement methods: four studies used water displacement to evaluate the incidence of upper extremity lymphedema, ${ }^{1,20,30,32}$ five studies measured the arm circumference and one study used a questionnaire survey ${ }^{17-19,35,39,47]}$ (Table 4.3).

Three of the ten studies reported an absence of upper extremity lymphedema in patients in whom the ARM lymph nodes were preserved compared to $13 \%$ (reported by Boneti et al.) and 6\% (reported by Han et al.) upper extremity lymphedema in the population who had an ARM lymph node resected. All of them had a short follow-up of 9 months or less (Table 4.3). However, since only 51 of the 182 patients had preand postoperative arm volumes recorded, conclusions with regard to the true incidence have to be interpreted with some caution. ${ }^{32}$ Furthermore, two studies reported a non-significant decrease in the incidence of upper extremity lymphedema in patients in whom the ARM lymph nodes were preserved (Table 4.3). ${ }^{1,39}$

Robust conclusions with regard to the clinical use of the ARM procedure for reducing upper extremity lymphedema from these studies are rather difficult to put forward due to small study size, different measurement methods, short follow-up and differences in the criteria of upper extremity lymphedema even when using the same measurement methods.

However, a recent prospective randomised study by Yue et al. reported on 265 patients with an average follow-up of 20 months. ${ }^{35}$ They found that upper extremity lymphedema occurred in $7(6 \%)$ patients in the ARM group compared to $42(33 \%)$ patients in the control group (Table 4.3). In another study by Pasko et al., who used a questionnaire survey, upper extremity lymphedema was reported by $27 \%$ of the ARM lymph node 'preserved group' compared to $50 \%$ of the 'removed group'. ${ }^{18}$

In conclusion, the ARM procedure may potentially significantly contribute to a reduction in upper extremity lymphedema in patients who undergo ALND. The ongoing randomised controlled multicentre trial in The Netherlands (ALND with or without preservation of ARM lymph nodes and corresponding lymphatics in SLN+ patients) will further contribute to the clinical utility of this procedure. ${ }^{48}$

\section{Conclusion}

In summary, by carrying out an ARM procedure using blue dye or fluorescence, upper extremity lymph nodes and lymphatics in the axillary basin can be identified in up to 
around $90 \%$. Preservation of the ARM lymph nodes and corresponding lymphatics is oncologically safe in clinically node-negative breast cancer patients with metastatic lymph node involvement in the SLN who are advised to undergo a completion ALND. In cases of clinical node-positive disease, proper selection of patients scheduled for completion axillary surgical treatment ARM-ALND) may be considered. This surgical refinement of ALND may reduce the incidence of upper extremity lymphedema. 


\section{References}

1. Tausch C, Baege A, Dietrich D, Vergin I, Heuer H, Heusler RH, et al. Can axillary reverse mapping avoid lymphedema in node positive breast cancer patients? Eur J Surg Oncol. 2013;39:880-6.

2. Noguchi M, Morioka E, Ohno Y, Noguchi M, Nakano Y, Kosaka T. The changing role of axillary lymph node dissection for breast cancer. Breast Cancer. 2013;20:41-6.

3. Blanchard DK, Donohue JH, Reynolds C, Grant CS. Relapse and morbidity in patients undergoing sentinel lymph node biopsy alone or with axillary dissection for breast cancer. Arch Surg. 2003;138:482-7.

4. Haid A, Koberle-Wuhrer R, Knauer M, Burtscher J, Fritzsche H, Peschina W, et al. Morbidity of breast cancer patients following complete axillary dissection or sentinel node biopsy only: a comparative evaluation. Breast Cancer Res Treat. 2002;73:31-6.

5. Leidenius M, Leivonen M, Vironen J, von Smitten K. The consequences of long-time arm morbidity in node-negative breast cancer patients with sentinel node biopsy or axillary clearance. J Surg Oncol. 2005;92:23-31.

6. Mansel RE, Fallowfield L, Kissin M, Goyal A, Newcombe RG, Dixon JM, et al. Randomized multicenter trial of sentinel node biopsy versus standard axillary treatment in operable breast cancer: the ALMANAC Trial. J Natl Cancer Inst. 2006;98:599-609.

7. Ronka R, von Smitten $K$, Tasmuth $T$, Leidenius M. One-year morbidity after sentinel node biopsy and breast surgery. Breast. 2005;14:28-36.

8. Schijven MP, Vingerhoets AJ, Rutten HJ, Nieuwenhuijzen GA, Roumen RM, van Bussel ME, et al. Comparison of morbidity between axillary lymph node dissection and sentinel node biopsy. Eur J Surg Oncol. 2003;29:341-50.

9. Schrenk P, Rieger R, Shamiyeh A, Wayand W. Morbidity following sentinel lymph node biopsy versus axillary lymph node dissection for patients with breast carcinoma. Cancer. 2000;88:608-14.

10. Swenson KK, Nissen MJ, Ceronsky C, Swenson L, Lee MW, Tuttle TM. Comparison of side effects between sentinel lymph node and axillary lymph node dissection for breast cancer. Ann Surg Oncol. 2002;9:745-53.

11. Noguchi M, Miwa K, Michigishi T, Yokoyama K, Nishijima H, Takanaka T, et al. The Role of Axillary Lymph Node Dissection in Breast Cancer Management. Breast Cancer. 1997;4:143-53.

12. Thompson M, Korourian S, Henry-Tillman R, Adkins L, Mumford S, Westbrook KC, et al. Axillary reverse mapping (ARM): a new concept to identify and enhance lymphatic preservation. Ann Surg Oncol. 2007;14:1890-5.

13. Nos C, Lesieur B, Clough KB, Lecuru F. Blue dye injection in the arm in order to conserve the lymphatic drainage of the arm in breast cancer patients requiring an axillary dissection. Ann Surg Oncol. 2007; 14:2490-6.

14. Pavlista D, Eliska O. Analysis of direct oil contrast lymphography of upper limb lymphatics traversing the axilla -- a lesson from the past -- contribution to the concept of axillary reverse mapping. European journal of surgical oncology : the journal of the European Society of Surgical Oncology and the British Association of Surgical Oncology 2012;38:390-4.

15. Hama Y, Koyama Y, Urano Y, Choyke PL, Kobayashi H. Simultaneous two-color spectral fluorescence lymphangiography with near infrared quantum dots to map two lymphatic flows from the breast and the upper extremity. Breast Cancer Res Treat. 2007;103:23-8.

16. Deng H, Chen L, Jia W, Chen K, Zeng Y, Rao N, et al. Safety study of axillary reverse mapping in the surgical treatment for breast cancer patients. J Cancer Res Clin Oncol. 2011; 137:1869-74.

17. Han JW, Seo YJ, Choi JE, Kang SH, Bae YK, Lee SJ. The efficacy of arm node preserving surgery using axillary reverse mapping for preventing lymphedema in patients with breast cancer. J Breast Cancer. 2012;15:91-7.

18. Pasko JL, Garreau J, Carl A, Ansteth M, Glissmeyer M, Johnson N. Axillary reverse lymphatic mapping reduces patient perceived incidence of lymphedema after axillary dissection in breast cancer. Am J Surg. 2015;209:890-5 
19. Casabona F, Bogliolo S, Valenzano Menada M, Sala P, Villa G, Ferrero S. Feasibility of axillary reverse mapping during sentinel lymph node biopsy in breast cancer patients. Ann Surg Oncol. 2009;16: 2459-63.

20. Ochoa D, Korourian S, Boneti C, Adkins L, Badgwell B, Klimberg VS. Axillary reverse mapping: five-year experience. Surgery. 2014;156:1261-8.

21. Kuusk U, Seyednejad N, McKevitt EC, Dingee CK, Wiseman SM. Axillary reverse mapping in breast cancer: a Canadian experience. J Surg Oncol. 2014;110:791-5.

22. Clough KB, Nasr R, Nos C, Vieira M, Inguenault C, Poulet B. New anatomical classification of the axilla with implications for sentinel node biopsy. Br J Surg. 2010;97:1659-65.

23. Bedrosian I, Babiera GV, Mittendorf EA, Kuerer HM, Pantoja L, Hunt KK, et al. A phase I study to assess the feasibility and oncologic safety of axillary reverse mapping in breast cancer patients. Cancer. 2010;116:2543-8.

24. Nos C, Kaufmann G, Clough KB, Collignon MA, Zerbib E, Cusumano $P$, et al. Combined axillary reverse mapping (ARM) technique for breast cancer patients requiring axillary dissection. Ann Surg Oncol. 2008;15:2550-5.

25. Connor C, McGinness M, Mammen J, Ranallo L, Lafaver S, Klemp J, et al. Axillary reverse mapping: a prospective study in women with clinically node negative and node positive breast cancer. Ann Surg Oncol. 2013;20:3303-7.

26. Khandelwal R, Poovamma CU, Shilpy C, Prema M, Anthony P. Axillary reverse mapping: Is it feasible in locally advanced breast cancer patients? Breast Dis. 2014;34:151-5.

27. Ponzone R, Cont NT, Maggiorotto F, Cassina E, Mininanni P, Biglia N, et al. Extensive nodal disease may impair axillary reverse mapping in patients with breast cancer. J Clin Oncol. 2009;27:5547-51.

28. Schunemann E, Jr., Doria MT, Silvestre JB, Gasperin P, Jr., Cavalcanti TC, Budel VM. Prospective study evaluating oncological safety of axillary reverse mapping. Ann Surg Oncol. 2014;21: 2197-202.

29. Rubio IT, Cebrecos I, Peg V, Esgueva A, Mendoza C, Cortadellas T, et al. Extensive nodal involvement increases the positivity of blue nodes in the axillary reverse mapping procedure in patients with breast cancer. J Surg Oncol. 2012;106:89-93.

30. Boneti C, Badgwell B, Robertson Y, Korourian S, Adkins L, Klimberg V. Axillary reverse mapping (ARM): initial results of phase II trial in preventing lymphedema after lymphadenectomy. Minerva Ginecol. 2012;64:421-30.

31. Boneti C, Korourian S, Bland K, Cox K, Adkins LL, Henry-Tillman RS, et al. Axillary reverse mapping: mapping and preserving arm lymphatics may be important in preventing lymphedema during sentinel lymph node biopsy. J Am Coll Surg. 2008;206:1038-42.

32. Boneti C, Korourian S, Diaz Z, Santiago C, Mumford S, Adkins L, et al. Scientific Impact Award: Axillary reverse mapping (ARM) to identify and protect lymphatics draining the arm during axillary lymphadenectomy. Am J Surg. 2009;198:482-7.

33. Gobardhan PD, Wijsman JH, van Dalen T, Klompenhouwer EG, van der Schelling GP, Los J, et al. ARM: axillary reverse mapping - the need for selection of patients. Eur J Surg Oncol. 2012;38:657-61.

34. Klimberg VS. A new concept toward the prevention of lymphedema: axillary reverse mapping. J Surg Oncol. 2008;97:563-4.

35. Yue T, Zhuang D, Zhou P, Zheng L, Fan Z, Zhu J, et al. A Prospective Study to Assess the Feasibility of Axillary Reverse Mapping and Evaluate Its Effect on Preventing Lymphedema in Breast Cancer Patients. Clinical Breast Cancer. 2015;15:301-6.

36. Gobardhan PD, Klompenhouwer EG, Beek MA, Voogd AC, Luiten EJ. [Axillary reverse mapping preserving nodes during an axillary lymph node dissection]. Ned Tijdschr Geneeskd. 2013;157:A5646.

37. Noguchi M, Yokoi M, Nakano Y. Axillary reverse mapping with indocyanine fluorescence imaging in patients with breast cancer. J Surg Oncol. 2010;101:217-21.

38. Noguchi M, Noguchi M, Nakano Y, Ohno Y, Kosaka T. Axillary reverse mapping using a fluorescence imaging system in breast cancer. J Surg Oncol. 2012;105:229-34

39. Ikeda K, Ogawa Y, Kajino C, Deguchi S, Kurihara S, Tashima T, et al. The influence of axillary reverse mapping related factors on lymphedema in breast cancer patients. Eur J Surg Oncol. 2014;40:818-23. 
41. Ikeda K, Ogawa Y, Komatsu H, Mori Y, Ishikawa A, Nakajima T, et al. Evaluation of the metastatic status of lymph nodes identified using axillary reverse mapping in breast cancer patients. World J Surg Oncol. 2012;10:233.

42. Alvarado R, Yi M, Le-Petross H, Gilcrease M, Mittendorf EA, Bedrosian I, Hwang RF, Caudle AS, Babiera GV, Akins JS, Kuerer HM, Hunt KK. The role for sentinel lymph node dissection after neoadjuvant chemotherapy in patients who present with node-positive breast cancer. Annals of surgical oncology 2012;19:3177-84.

43. Beek MA, Gobardhan PD, Klompenhouwer EG, Rutten HJ, Voogd AC, Luiten EJ. Axillary reverse mapping (ARM) in clinically node positive breast cancer patients. Eur J Surg Oncol. 2015;41:59-63.

44. Beek MA, Tetteroo E, Luiten EJ, Gobardhan PD, Rutten HJ, Heijns JB, Voogd AC, Klompenhouwer EG. Clinical impact of breast MRI with regard to axillary reverse mapping in clinically node positive breast cancer patients following neo-adjuvant chemotherapy. Eur J Surg Oncol. 2016;42:672-8.

45. Coen JJ, Taghian AG, Kachnic LA, Assaad SI, Powell SN. Risk of lymphedema after regional nodal irradiation with breast conservation therapy. Int J Radiat Oncol Biol Phys. 2003;55:1209-15.

46. Britton TB, Solanki CK, Pinder SE, Mortimer PS, Peters AM, Purushotham AD. Lymphatic drainage pathways of the breast and the upper limb. Nucl Med Commun. 2009;30:427-30.

47. Gennaro M, Maccauro M, Sigari C, Casalini P, Bedodi L, Conti AR, et al. Selective axillary dissection after axillary reverse mapping to prevent breast-cancer-related lymphoedema. Eur J Surg Oncol. 2013;39:1341-5.

48. Klompenhouwer EG, Gobardhan PD, Beek MA, Voogd AC, Luiten EJ. The clinical relevance of axillary reverse mapping (ARM): study protocol for a randomized controlled trial. Trials. 2013;14:111. 



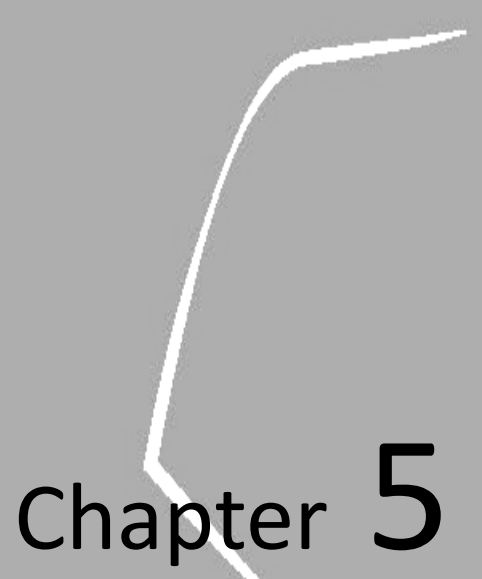

The clinical relevance of axillary reverse mapping (ARM): study protocol for a randomized

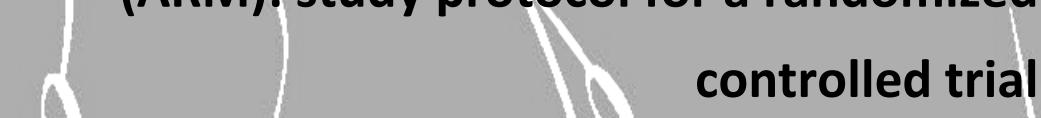




\section{Abstract}

\section{Background}

Axillary lymph node dissection for patients with breast cancer has the potential to induce side effects, including upper extremity lymphedema. Axillary reverse mapping (ARM) is a technique that enables discrimination of lymphatic drainage of the breast from the upper extremity in the axillary lymph node basin. If lymphedema is caused by removing these lymphatics and nodes of the upper extremity, the possibility to identify these lymphatics would enable surgeons to preserve them. The aim of this study is to determine the clinical relevance of selective axillary lymph node and lymphatics preservation by means of ARM. To minimize the risk of overlooking tumorpositive ARM nodes and the associated risk of under treatment, we will only include patients with a tumor-positive sentinel lymph node. Patients who are candidates for axillary lymph node dissection because of a proven positive axilla at clinical examination can be included in a registration study.

\section{Methods and design}

280 Patients diagnosed with sentinel lymph node biopsy metastasis of invasive breast cancer with an indication for a completion axillary lymph node dissection will be included in the study. Patients will be randomized between a standard axillary lymph node dissection or an axillary lymph node dissection in which the ARM nodes and their corresponding lymphatics will be left in situ. Primary outcome is the presence of axillary surgery related lymphedema at 6, 12 and 24 months postoperatively, measured by the water displacement method. Secondary outcome measures include: pain, paresthesia, numbness and loss of shoulder mobility, quality of life and axillary recurrence risk.

\section{Discussion}

The benefit of an axillary lymph node dissection in patients with a positive sentinel node is under debate. For many patients an axillary lymph node dissection will remain to be the treatment of choice. This multicenter randomized trial will provide evidence on whether or not axillary lymph node preservation by means of ARM will decrease the side effects of an axillary lymph node dissection. The inclusion of patients will start in January 2013 in five breast cancer centers in the Netherlands and is expected to close by January 2016. 


\section{Background}

Axillary lymph node dissection (ALND) for patients with breast cancer has the potential to cause side-effects, including pain, numbness or paresthesia, arm/shoulder mobility restriction and lymphedema. ${ }^{1}$ Arm lymphedema is documented in $7-77 \%$ of patients who undergo ALND. ${ }^{2-3}$ The incidence of lymphedema depends on whether ALND has been combined with subsequent radiotherapy, but also on the definition used.

Sentinel lymph node biopsy (SLNB) represents the standard of care for axillary node staging in patients with early-stage, clinically node-negative breast cancer (cT1-2N0). The goals of SLNB are to reduce the morbidity of breast cancer surgery by avoiding an unnecessary ALND and to improve staging of the regional lymph node basis. However, in a selected group of patients ALND is still indicated.

Axillary reversed mapping (ARM) is a recently developed technique that enables surgeons to discriminate the lymphatic drainage pattern from the breast and the arm. The concept of ARM is to map the drainage of the arm to determine the anatomical variation in these lymphatics in order to create a roadmap for their preservation. If lymphedema is caused by removing these lymphatics and nodes of the upper extremity, the possibility to identify these lymphatics would enable surgeons to preserve them. ${ }^{4}$ The ARM technique can be performed by using blue dye, fluorescence or a radioisotope. In the past few years several groups have reported their first experiences with this relatively new technique. ${ }^{5-13} \mathrm{~A}$ review by Ngochi on this topic ${ }^{14}$ clearly described the ARM techniques that are currently available. Studies on ARM using blue dye reported identification rates varying between $50-89 \%,{ }^{5-10}$ and for isotope and fluorescence these rates are $91-100 \%{ }^{11,13}$ and $88 \%{ }^{12}$ respectively. The latter two visualization techniques have the advantage of not leaving a 'blue tattoo' on the patients' arm. On the other hand, there is limited experience with the use of isotopes and fluorescence and they require the use of expensive equipment during surgery.

Between October 2009 and June 2011 we performed a pilot study using blue dye for visualizing lymph nodes drainage of the upper extremity. ${ }^{15}$ Patients with invasive breast cancer and an indication for ALND were enrolled in the study, including 50 patients with a tumor-positive SLN and 43 patients who had axillary metastases proven by preoperative cytology. During surgery ARM-nodes were identified and removed first, followed by ALND (at least level I-II). No significant differences were observed in visualization rate of ARM-nodes between the groups (86 vs. $94 \%$ respectively, $P=0.196)$. In the group of patients with a positive SLN none of the ARMnodes contained metastases, as compared to 11 (22\%) of the ARM-nodes in the group with axillary metastases proven by preoperative cytology $(P=0.001)$. Patients receiving 
neoadjuvant chemotherapy had a significantly lower risk of additional axillary lymph node metastases ( 24.6 vs. $44.4 \%, P=0.046)$. Our results are largely in accordance with other studies. Boneti et al., ${ }^{8}$ Thompson et al. ${ }^{7}$ and Casbona et al. ${ }^{10}$ found no tumor deposits in the ARM nodes even when the patients had positive axillary nodes in the initial series. However, Nos et al. ${ }^{6}$ found metastases in $14 \%$ of the ARM nodes ( 3 out of 21 patients), all being associated with extensive axillary lymph node metastasis. Noguchi et al. ${ }^{12}$ found ARM node metastases in 3 out of 7 patients who underwent ALND, and all three patients had a clinically positive axilla (N1/N2). Based on our results and those from the literature, we conclude that the ARM procedure using blue dye, is technically feasible and has a high visualization rate and that its use might be considered in patients with a positive SLN. Still, more research is needed to determine the safety of ARM in patients with clinically positive lymph nodes.

Despite these promising results, there are some problems that need to be considered before ARM can be used in routine clinical practice. In some patients the available techniques are insufficient to identity the arm lymph nodes. The relevance of the ARM procedure is based on the assumption that the lymphatic pathways from the upper extremity are not involved in metastasis of the primary breast cancer. ${ }^{5}$ However, the SLN draining of the breast may be the same as the ARM node draining the upper extremity in a minority of patients. This may explain why the ARM nodes may be involved with metastatic foci in patients with clinically axillary lymph node metastases. These issues may represent an important drawback for the implementation of the ARM procedure.

Boneti et al. recently published a phase II trial with promising results. ${ }^{16}$ He shows that preserving the ARM nodes in a clinically negative axilla is safe and results in a low incidence of postoperative lymphedema in patients undergoing ALND and SLNB in a phase II trial. However, further studies are needed before this technique can be adopted as a standard procedure during CALND in surgical treatment of breast cancer.

In this paper we will present the design of a multicenter randomized controlled trial to determine the clinical relevance and safety of selectively sparing upper extremity axillary lymph nodes and their corresponding lymphatics by means of ARM. To minimize the risk of undertreatment due to non-removal of possible ARM node metastases, we will only include patients based on a tumor-positive sentinel lymph node.

\section{Design}

The aim of the ARM-trial is to determine the usefulness of the ARM technique in identifying and sparing the upper extremity related axillary lymph nodes and its ability to reduce the risk of lymphedema of the upper extremity. After determining the indication for a complementary axillary lymph node dissection (cALND) basis on a 
tumor-positive sentinel node, patients will be randomly allocated in two groups: ALND with preservation of the upper extremity related axillary lymph nodes (ALND-ARM) and a level I-II ALND (ALND-standard).

Patients who are candidates for a primary axillary lymph node dissection (pALND) based on a axillary metastases proven by preoperative cytology can be included in the registration study to confirm the feasibility of the procedure and to perform a further subgroup analysis. A schematic overview of the trial is given in Figure 5.1.

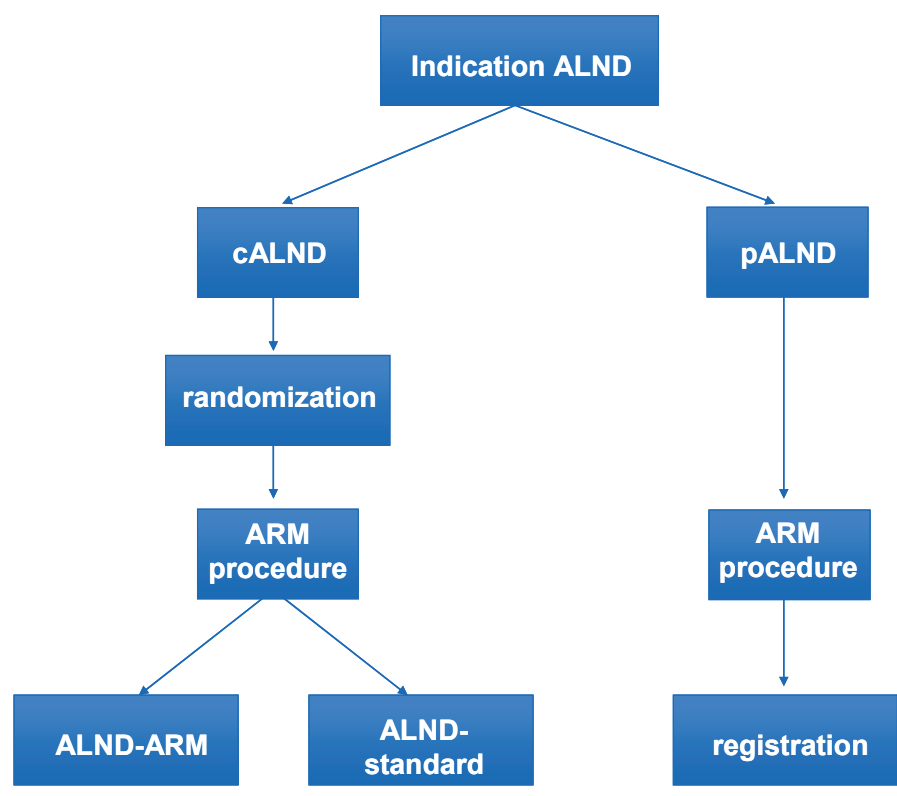

Figure 5.1 Flow chart of axillary reverse mapping (ARM) trial. ALND, Axillary lymph node dissection; CALND, complementary axillary lymph node dissection based on a tumor-positive sentinel node; PALND, primary axillary lymph node dissection based on a tumor-positive axilla proven by pre-operative cytology; ARM-ALND axillary lymph node dissection with preservation of the upper limb-related axillary lymph nodes; standard ALND, level I-II axillary lymph node dissection.

\section{Participants}

Female patients over 18 years presenting with invasive breast cancer and an indication for a cALND based on a positive SLN will be deemed eligible for the trial. The indication for the ALND should be made in a multidisciplinary team including an oncologic surgeon, a medical oncologist, a pathologist, a radiologist and a radiotherapist. Informed consent will be obtained from all patients. 
Exclusion criteria are:

- Indication for a primary ALND based on a clinical positive axilla;

- A contra-indication for SLNB;

- An adverse event during the previous SLNB;

- A history of breast cancer;

- A history of axillary surgery;

- Pregnancy.

Patients who undergo an ALND based on a clinical positive axilla can participate in the registration study, in which patients will undergo ARM during ALND. In this group of patients no attempts will be made to spare nodes or lymphatics.

\section{Objectives}

The hypothesis is that patients undergoing an ALND-ARM have less postoperative complications compared to patients undergoing an ALND-standard. The postoperative complications that will be measured are the occurrence of breast cancer related lymphedema (BCRL) and paresthesia/numbness, pain and shoulder immobility. The hypothesis is that a reduction of the risk of post-operative complications in patients undergoing ALND-ARM will result in a better quality of life as compared to those undergoing ALND-standard. Based on the results of our feasibility study, in which no lymph node metastasis where found in the patients with a tumor-positive SLN, it is expected that the axillary recurrence risk in the ALND-ARM group will be comparable to the risk in the ALND-standard group.

\section{Research questions}

1. Will there be less postoperative complications after ALND-ARM compared to standard-ALND in patients with an indication for a cALND based on a positive SLNB? Postoperative complications include:

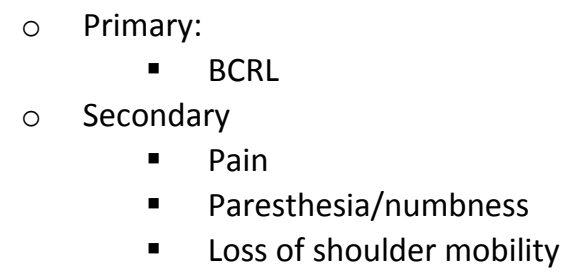

2. Do possible reductions of these postoperative complaints have a positive effect on the quality of life?

3. Do patients with an ALND-ARM have a similar axillary recurrence risk as the patients undergoing ALND-standard? 


\section{Randomization}

Randomization will be performed by using a web-based randomization system, and will be stratified by center. This randomization application and the web-help will be available 24 hours a day, seven days a week. Because of pratical considerations the randomization will take place as soon as the patient has given written informed consent. Based on our previous experience with the ARM-procedure we expect that identification or preservation of the ARM-nodes/lymphatics will not be possible in $5-15 \%$ of the randomized patients. Once this happens, a standard ALND will be performed, if possible, the ARM nodes/lymphatics will be separately removed and sent for analysis. These patients will be included in the analysis, according to the intention-to-treat principle, but excluded in a sensitivity analysis. (Figure 5.1, more information can be found on www.armstudie.nl)

\section{Interventions}

Surgeons familiar with the ARM-procedure will perform the ALND. In our feasibility study (Amphia hospital Breda, the Netherlands) we hardly measured a learning curve in performing the ARM-procedure and we accomplished an overall identification rate of the upper extremity lymph nodes up to $94 \%$. Based on these results we consider an oncologic breast surgeon capable of performing an ARM-procedure when 5 procedures have been performed under supervision of an oncologic surgeon from the Amphia Hospital Breda (EL/PG).

The procedure will be performed under general anesthesia. Approximately $2 \mathrm{ml}$ blue patent $V$ dye (Laboratoire Guerbet, Aulnay-sous-Bois, France) solution of $25 \mathrm{mg} / \mathrm{ml}$ (17) will be injected subcutaneously into the inner arm along the medial intermuscular groove of the ipsilateral arm. This dye is identical to the dye used to perform an SLNB. After injection, the site of injection is massaged and the arm will be elevated for a few minutes to enhance arm lymphatic drainage. Next, the patient is covered sterile and the ALND can start.

The technical part of the operation is identical to a normal level I-II ALND, accept for the first part of the operation. After incision, the blue painted lymph nodes and lymphatics are identified. In the ALND-ARM group, the blue painted lymph nodes and lymphatics will be spared, then a normal ALND will be performed. In the ALNDstandard group, a normal ALND will be performed without sparing the upper extremity lymphatics and nodes. The pathologist will separately analyze the lymph nodes (level I-II nodes and ARM-nodes, Figure 5.2). 


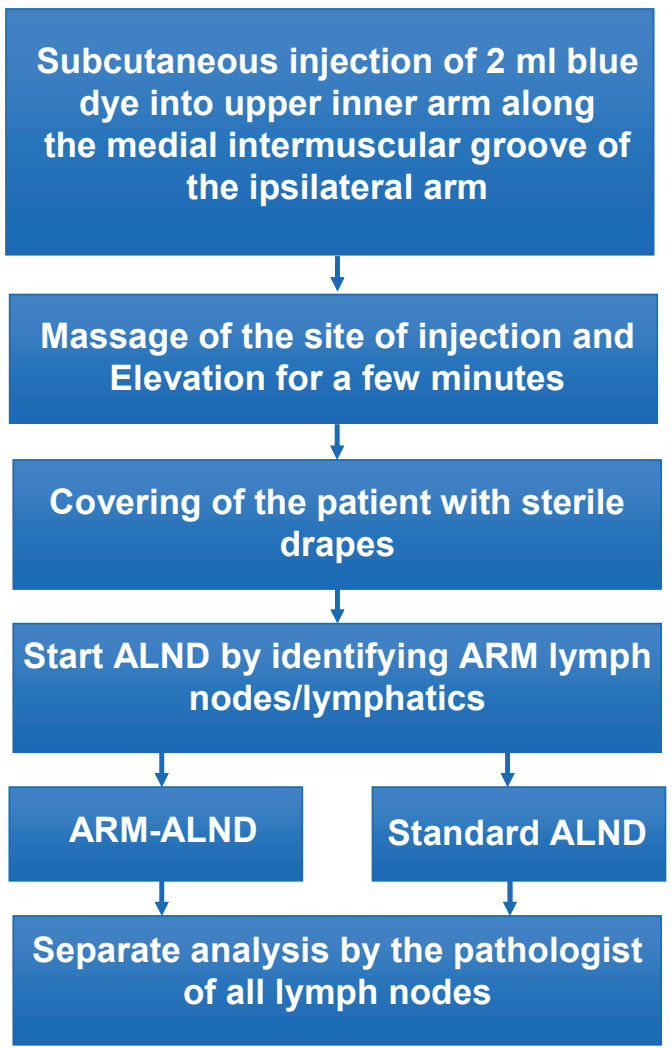

Figure 5.2 Axillary reverse mapping (ARM) procedure.

\section{Settings and location}

The study is a multicenter randomized controlled trial, which will be conducted in the Netherlands. Already 5 dedicated breast cancer centers are willing to participate:

- Amphia Hospital, Breda

- Catharina Hospital, Eindhoven

- Haga Hospital, The Hague

- Hospital Gelderse Vallei, Ede

- Albert Schweitzer Hospital, Dordrecht

Patients eligible for inclusion are enrolled by the breast surgeon at the outpatient clinic, following informed consent. 


\section{Primary and secondary outcome measures}

Our primary outcome is the occurrence of lymphedema in the upper extremity. Lymphedema will be measured as follows at 6, 12 and 24 months:

a) The water displacement method. This method is regarded as the gold standard for the measurement of lymphedema. For the assessment of upper-limb volume an arm volume meter will be used $\left(\right.$ Varitex $\left.{ }^{18}\right)$. This method has a high reproducibility. ${ }^{19}$ Both upper-limbs will be measured pre- and postoperatively. Lymphedema is defined as a limb volume difference greater than $200 \mathrm{~mL}$ between the treated and untreated side, according to the study by Lopez Penha et al. ${ }^{20}$

b) Circumference method. The arm circumference will be measured at 4 sites: metacarpophalangeal joints, wrist, $10 \mathrm{~cm}$ distal of the lateral epicondyle en $15 \mathrm{~cm}$ proximal of the epicondyle. A difference of more than $2 \mathrm{~cm}$ between the affected and contra-lateral arm is considered clinically significant.

Secondary end-points are measured at 6, 12 and 24 months and include:

a) Paresthesia/numbness: These outcomes will be documented with the aid of a standardized questionnaire developed for breast cancer patients ${ }^{21}$

b) Pain: This will be measured through a VAS-score.

c) Loss of shoulder mobility: The shoulder function of the affected upper extremity will be compared with the not affected upper extremity. Loss of abduction of more than 20 degrees is regarded as abnormal.

d) Quality of life: These outcomes will be documented with the aid of a standardized questionnaire developed for breast cancer patients. ${ }^{21}$ This questionnaire includes the WHOQOL-Bref.

e) Loco-regional recurrence rate: This will be checked during follow-up by means of physical examination. In case of suspicion, an ultrasound (with or without biopsy) will be performed.

\section{Data collection}

A nurse practitioner or clinical investigator (depending on the local organization of the participating hospital) will be responsible for the preoperative and postoperative data collection. The surgeon who performed the ALND will be responsible for the perioperative data collection. Data will be stored anonymously by using a unique trial number. The principal investigators (EL/PG) will have access to the data. All data will be collected in an online CRF and an online participant questionnaire (PQ). The timetable and source of the data collection is listed in Table 5.1. 
Table 5.1 Data collection.

\begin{tabular}{lccc}
\hline Data & Source & Preoperative & $\begin{array}{c}\text { Perioperative } \\
\text { Follow-up at 6, 12, } \\
\text { and 24 months }\end{array}$ \\
\hline Baseline characteristics & CRF & $\mathrm{X}$ & \\
Medical history & CRF & $\mathrm{X}$ & \\
Pre-operative tumor status/ markers & CRF & $\mathrm{X}$ & $\mathrm{X}$ \\
Operation data including: & CRF & & \\
Registration & & & $\mathrm{X}$ \\
Randomization & & $\mathrm{X}^{\text {a }}$ & $\mathrm{X}$ \\
Post-operative complications & $\mathrm{CRF} / \mathrm{PQ}$ & & $\mathrm{X}$ \\
Quality of life & $\mathrm{PQ}$ & & \\
Axillary recurrence rate & $\mathrm{CRF}$ & & \\
\hline
\end{tabular}

$\mathrm{CRF}$, case report form; $\mathrm{PQ}$, participant questionnaire; ${ }^{\mathrm{a}}$ The volume, circumference and mobility of the arm will be measured pre-operatively.

\section{Blinding}

The qualified oncologic breast surgeon will perform the procedure and cannot be blinded. The patient as well as the person responsible for the postoperative data collection and measurements will not be aware of the performed procedure (double blind).

\section{Statistics}

\section{Sample size}

The clinically relevant amount of lymphedema is stated as a volume difference of $200 \mathrm{ml}$ or more between both arms. Primary research shows that the risk of BCRL is $15 \%$ in patients undergoing an ALND. It is expected that ALND-ARM will reduce the risk to $5 \%$. Based on these assumptions we will need to study 140 subjects in the ALND-standard and 140 subjects in the ALND-ARM group to be able to reject the null hypothesis that the failure rates for experimental and control subjects are equal with probability (power) 0.8 . The Type I error probability associated with this test of this null hypothesis is 0.05 .

\section{Data analysis}

We will use an uncorrected chi-squared statistic to test the null hypothesis regarding the proportion of patients developing lymphedema in the upper extremity. These tests will be performed after 6, 12 and 24 months. We are interested in the course of the development of lymph edema, based on the measurements at each of the three specified time-point. As lymph edema is known to develop at least until 2 years after surgery, or even longer, the measurement at 24 months is considered our primary end-point. 
To compare continuous data, like pain and quality of life, analyses of co-variance (ANCOVA) will be performed, using baseline values as a co-variate. Separate analyses will be performed for the three time-points $(6,12$ and 24 months). The local recurrence rate in both groups will be analyzed with the Kaplan-Meier method and compared with the logrank-test. A repeated measurement analysis will be performed, to compare the changes in continuous data between the two groups, until two year after treatment. It is expected that not only the prevalence of lymphedema will be higher in the ALND-standard arm, but also that it will occur earlier and will be more severe. To test this hypothesis, interaction terms between treatment and time will be included in the repeated measures analysis.

All data will be documented and analyzed in SPSS, with the exception of the repeated measurements. These will be run in SAS with the procedure PROC MIXED. No interimanalysis will be performed.

\section{Discussion}

Treatment of the axilla in breast cancer patients is under debate recently. More than $50 \%$ of the patients with a positive SLN appear to have no further lymph node involvement. ${ }^{22-23}$ Based on these findings, the therapeutic role of a cALND for these patients, seems limited. ${ }^{24}$

In the late 1990s the ACOSOG Z0011 trial started in the United States. This trial randomized breast cancer patients with one to three positive SLN's between a complementary ALND and a 'wait and see' policy. The recently published results showed no differences in overall and disease free survival with a median follow up of 6.3 years. ${ }^{25}$ Although the results do question the therapeutic benefit of ALND in SLNB positive patients, abandoning ALND is not yet regarded as standard of care. Moreover, considering the selected group of patients in the ACOSOG Z0011 trial of which the majority received adjuvant chemotherapy as well as locoregional radiotherapy of the breast with inclusion of lower regions of the axilla, the safety of omitting ALND in patients not fulfilling these criteria remains unclear.

Furthermore, a recently published study by Avril et al. ${ }^{26}$ failed to demonstrate benefit in event free and overall survival for post-menopausal early breast cancer patients. This study randomized clinically node negative post-menopausal breast cancer patients between ALND and no ALND. Adjuvant systemic therapy was based on the primary tumor characteristics.

In studies, published several years ago, no difference was found in loco regional disease control, metastatic free survival and overall survival when comparing ALND with radiotherapy of the axilla. ${ }^{27}$ However, as is the case with standard ALND, 
axillary radiotherapy appeared to be associated with an increased incidence of lymphedema. ${ }^{28,29}$

The recently published Dutch guideline for breast cancer treatment ${ }^{30}$ leaves several therapeutic options for the treatment of the axilla. It says that, in case of micrometastasis and macrometastasis with a maximum of 2 positive SLN in patients receiving a breast sparing therapy in combination with adjuvant systemic therapy, omitting of the cALND can be considered. This may affect the accrual of the trial. However, there will be many patients left in which a primary and cALND will remain the treatment of first choice, for example in patients undergoing a mastectomy or in patients with a high risk of axillary involvement. For these patients, a limited and more tailor-made ALND based on the ARM technique, might be a valuable tool in order to reduce the morbidity associated with the current ALND technique.

This multicenter randomized trial will provide evidence on whether or not upper extremity axillary lymph node preservation by means of ARM will decrease the side effects of an ALND. 


\section{References}

1. Hack TF, Cohen L, Katz J, Robson LS, Goss P. Physical and psychological morbidity after axillary lymph node dissection for breast cancer. J Clin Oncol. 1999;17:143-9.

2. Schijven MP, Vingerhoets AJ, Rutten HJ, Nieuwenhuijzen GA, Roumen RM, van Bussel ME, et al. Comparison of morbidity between axillary lymph node dissection and sentinel node biopsy. Eur J Surg Oncol. 2003;29:341-50.

3. Ronka R, von Smitten K, Tasmuth $T$, Leidenius M. One-year morbidity after sentinel node biopsy and breast surgery. Breast. 2005;14:28-36.

4. Klimberg VS. A new concept toward the prevention of lymphoedema: axillary reverse mapping. J Surg Oncol. 2008;97:563-4.

5. Ponzone R, Mininanni P, Cassina E, Sismondi P. Axillary reverse mapping in breast cancer: can we spare what we find? Ann Surg Oncol. 2008;15:390-1.

6. Nos C, Lesieur B, Clough KB, Lecuru F. Blue dye injection in the arm in order to conserve the lymphatic drainage of the arm in breast cancer patients requiring an axillary dissection. Ann Surg Oncol. 2007; 14:2490-6.

7. Thompson M, Korourian S, Henry-Tillman R, Adkins L, Mumford S, Westbrook KC, et al. Axillary reverse mapping (ARM): a new concept to identify and enhance lymphatic preservation. Ann Surg Oncol. 2007;14:1890-5.

8. Boneti C, Korourian S, Bland K, Cox K, Adkins LL, Henry-Tillman RS, et al. Axillary reverse mapping: mapping and preserving arm lymphatics may be important in preventing lymphoedema during sentinel lymph node biopsy. J Am Coll Surg. 2008;206:1038-42.

9. Casabona F, Bogliolo S, Ferrero S, Boccardo F, Campisi C. Axillary reverse mapping in breast cancer: a new microsurgical lymphatic-venous procedure in the prevention of arm lymphoedema. Ann Surg Oncol. 2008;15:3318-9.

10. Casabona F, Bogliolo S, Valenzano Menada M, Sala P, Villa G, Ferrero S. Feasibility of axillary reverse mapping during sentinel lymph node biopsy in breast cancer patients. Ann Surg Oncol. 2009;16: 2459-63.

11. Nos C, Kaufmann G, Clough KB, Collignon MA, Zerbib E, Cusumano P, et al. Combined axillary reverse mapping (ARM) technique for breast cancer patients requiring axillary dissection. Ann Surg Oncol. 2008;15:2550-5.

12. Noguchi M, Yokoi M, Nakano Y. Axillary reverse mapping with indocyanine fluorescence imaging in patients with breast cancer. J Surg Oncol. 2010;101:217-21.

13. Britton TB, Solanki CK, Pinder SE, Mortimer PS, Peters AM, Purushotham AD. Lymphatic drainage pathways of the breast and the upper limb. Nucl Med Commun. 2009;30:427-30.

14. Noguchi M. Axillary reverse mapping for breast cancer. Breast Cancer Res Treat. 2010;119:529-

15. Gobardhan PD, Wijsman JH, van Dalen T, Klompenhouwer EG, van der Schelling GP, Los J, Voogd AC, Luiten EJ. ARM: axillary reverse mapping - the need for selection of patients. Eur J Surg Oncol. 2012;38:657-61.

16. Boneti C, Badgwell B, Roberton Y, Korourian S, Adkins L, Klimberg V. Axillary reverse mapping (ARM): initial results of phase II trial in preventing lymphedema after lymphadenectomy. Minerva ginecol 2012;64:421-30.

17. www.guebert.nl

18. www.varitex.nl

19. Damstra RJ, Glazenbrug EJ, Hop WCJ. Validation of the inverse water volumetry method: a new gold standard for arm volume measurements. Breast Cancer Res Treat. 2006;99:267-23.

20. Lopez Penha TR, Slangen JJ, Heuts EM, Voogd AC, Von Meyenfeldt MF. Prevalence of lymphoedema more than five years after breast cancer treatment. Eur J Surg Oncol. 2011;37:1059-63.

21. Ververs JM, Roumen RM, Vingerhoets AJ, Vreugdenhil G, Coebergh JW, Crommelin MA, et al. Risk, severity and predictors of physical and psychological morbidity after axillary lymph node dissection for breast cancer. Eur J Cancer. 2001;37:991-9. 


\section{Chapter 5}

22. Kim T, Giuliano AE, Lyman GH. Lymphatic mapping and sentinel lymph node biopsy in early-stage breast carcinoma: a metaanalysis. Cancer. 2006;106:4-16.

23. Straver ME, Meijnen P, van Tienhoven G, van de Velde CJ, Mansel RE, Bogaerts J, et al. Role of axillary clearance after a tumor-positive sentinel node in the administration of adjuvant therapy in early breast cancer. J Clin Oncol. 2010;28:731-7.

24. Fisher B, Jeong JH, Anderson S, Bryant J, Fisher ER, Wolmark N. Twenty-five-year follow-up of a randomized trial comparing radical mastectomy, total mastectomy, and total mastectomy followed by irradiation. N Engl J Med. 2002;347:567-75.

25. Giuliano AE, Hunt KK, Ballman KV, Beitsch PD, Whitworth PW, Blumencranz PW, et al. Axillary dissection vs no axillary dissection in women with invasive breast cancer and sentinel node metastasis: a randomized clinical trial. JAMA. 2011;305:569-75.

26. Avril A, Le Bouedec G, Lorimier G, Classe JM, Tunon-de-Lara C, Giard S, et al. Phase III randomized equivalence trial of early breast cancer treatments with or without axillary clearance in postmenopausal patients results after 5 years of follow-up. Eur J Surg Oncol. 2011;37:563-70.

27. Fisher B, Wolmark N, Fisher ER, Deutsch M. Lumpectomy and axillary dissection for breast cancer: surgical, pathological, and radiation considerations. World J Surg. 1985;9:692-8.

28. Deutsch M, Land S, Begovic M, Sharif S. The incidence of arm edema in women with breast cancer randomized on the National Surgical Adjuvant Breast and Bowel Project study B-04 to radical mastectomy versus total mastectomy and radiotherapy versus total mastectomy alone. Int J Radiat Oncol Biol Phys. 2008;70:1020-4.

29. Sakorafas GH, Peros G, Cataliotti L, Vlastos G. Lymphoedema following axillary lymph node dissection for breast cancer. Surg Oncol. 2006;15:153-65.

30. http://www.oncoline.nl/mammacarcinoom 
Reliability of the inverse water volumetry method to measure the volume of the upper limb

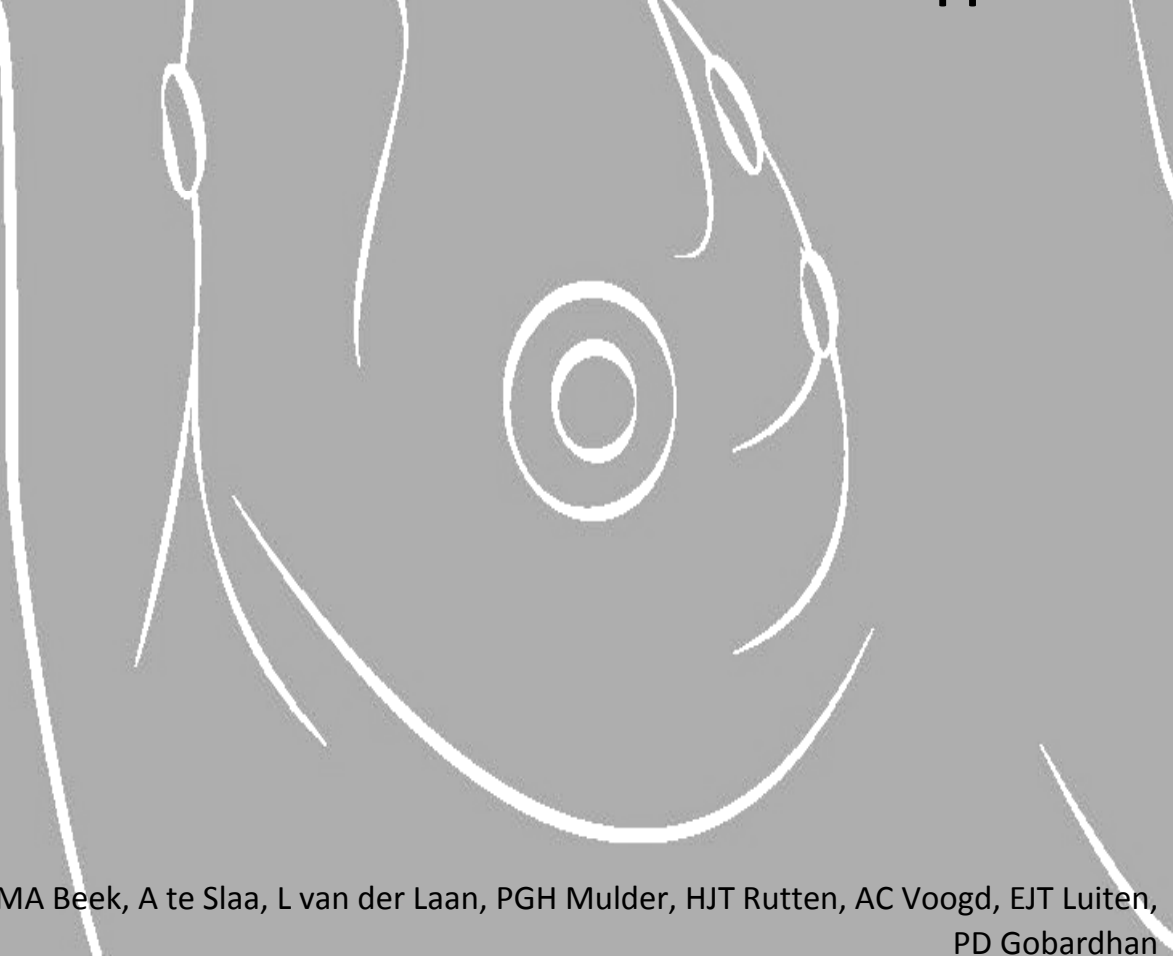

Lymphat Res Biol. 2015;13:126-30 


\section{Abstract}

\section{Background}

Lymphedema of the upper extremity is a common side effect of lymph node dissection or irradiation of the axilla. Several techniques are being applied in order to examine the presence and severity of lymphedema. Measurement of circumference of the upper extremity is most frequently performed. An alternative is the waterdisplacement method. The aim of this study was to determine the reliability and the reproducibility of the "Inverse Water Volumetry apparatus" (IWV-apparatus) for the measurement of arm volumes.

\section{Patients and methods}

The IWV-apparatus is based on the water-displacement method. Measurements were performed by three breast cancer nurse practitioners on ten healthy volunteers in three weekly sessions.

\section{Results}

The intra class correlation coefficient defined as the ratio of the subject component to the total variance equals 0.99 . The reliability index is calculated as $0.14 \mathrm{~kg}$. This indicates that only changes in a patient's arm volume measurement of more than $0.14 \mathrm{~kg}$ would represent a true change in arm volume, which is about $6 \%$ of the mean arm volume of $2.3 \mathrm{~kg}$.

\section{Conclusion}

The IWV-apparatus proved to be a reliable and reproducible method to measure arm volume. 


\section{Introduction}

Postoperative lymphedema of the upper extremity can be a significant problem for women treated for breast cancer. Lymphedema of the upper extremity is mostly seen after extensive axillary surgery (axillary lymph node dissection, ALND) but may also occur after minimal invasive axillary staging (sentinel lymph node biopsy, SLNB).

The incidence of arm lymphedema ranges from $0-13 \%$ in breast cancer patients who underwent a SLNB and from 7-77\% after ALND. ${ }^{1-9}$ This wide range is, mainly, due to the effect of the different definitions and measurement methods that are used to diagnose lymphedema. ${ }^{10}$ Commonly used methods to asses and to grade the presence of lymphedema are:

- the Herpertz method. ${ }^{11}$ This method is a combination of a four point circumference measurement of both limbs. Afterwards a calculation is made of the relative proportional swelling compared to the healthy side.

- circumference measurement at various levels on the both arms. A difference between both arms of $2.0 \mathrm{~cm}$ or more, or sometimes $2.5 \mathrm{~cm}$ or more, is used to define lymphedema.,12-16

- questionnaires to determine whether patients consider themselves as having lymphedema. ${ }^{6,8,17,18}$

Other methods based on perometry, ${ }^{19}$ ultrasonography, ${ }^{10}$ magnetic resonance imaging $^{20}$ and scintigraphy ${ }^{21}$ did not show to be viable in the current medical practice. Another, not widely used method is the "Inverse Water Volumetry" (IWV). This technique is based on the water-displacement method ${ }^{22-26}$ and is performed by using an IWV-apparatus (Figure 6.1). The water-displacement method is considered to be the most accurate method because it is easy to perform, painless and the possibility of direct measurement of objects with an irregular form. ${ }^{23-25,27}$ Currently, a multicentre trial is performed in the Netherlands with this IWV-apparatus to measure the presence of lymphedema. In this trial breast cancer patients undergoing axillary reverse mapping (ARM), a new type of axillary surgery which is expected to decrease upper extremity lymphedema. ${ }^{28}$ In this trial lymphedema is defined as a $10 \%$ difference between the pre-operative and the post-operative arm volume.

The aim of this study is to assess the reliability and the reproducibility of the IWVapparatus for measuring arm volumes.

\section{Patients and methods}

\section{Study cohort}

In this experiment ten healthy volunteers ( 5 males, 5 females) participated. All volunteers were medical students. Three observers (breast cancer nurse practitioners) 
performed all measurements and none of them acted as a subject or vice versa. All participants gave informed consent. Ethical approval for this study was provided by the local ethical commitee of the Amphia Hospital. The study was conducted in accordance with the Declaration of Helsinki.

\section{Inverse Water Volumetry apparatus ${ }^{29}$}

Archimedes' principle indicates that the upward buoyant force that is exerted on a body immersed in a fluid is equal to the weight of the fluid that the body displaces. The IWV-apparatus is based on Archimedes' principle (Figure 6.1).

Prior to use, calibration to zero is performed by filling the water tank till the reference point where the water flows into the overflow tube. The overflow tube is emptied where after the patient's arm is placed into the water tank. When the whole system is in equilibrium, patient's arm is removed and the display of the weighting device shows the shortness of water compared with the initial situation. Because the "lack of water" is measured, the method is named Inverse Water Volumetry". ${ }^{29}$

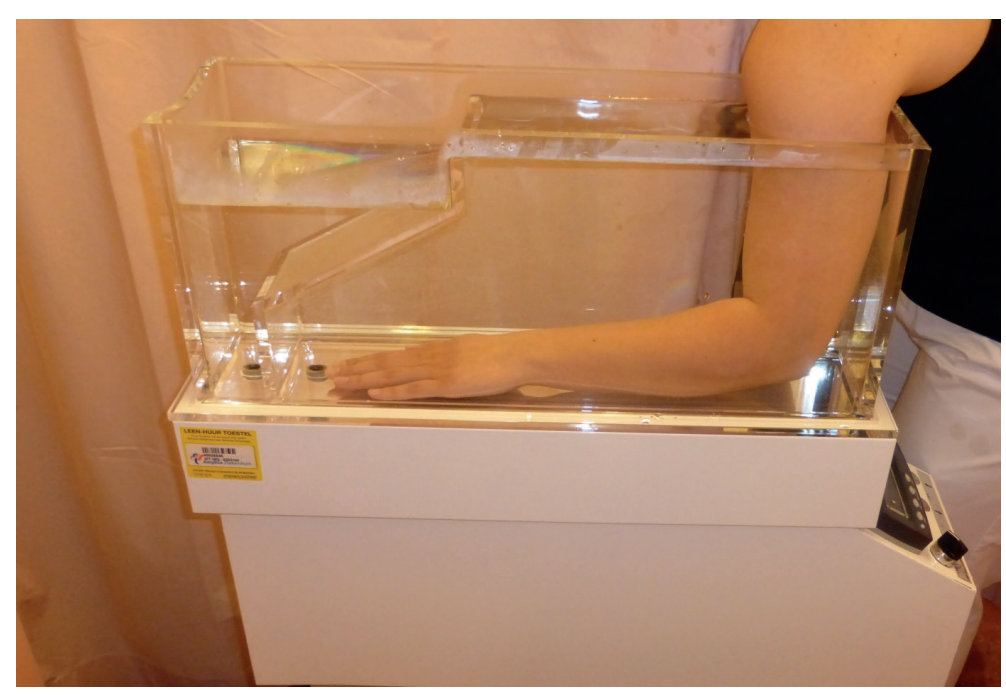

Figure 6.1 The Inverse Water Volumetry apparatus.

\section{Measurements}

The observers performed the arm volume measurements with the IWV-apparatus during three weekly sessions. At each session the subjects were separately measured by each of the three observers. Sessions were scheduled at the same time of day for 
each subject. The subjects were measured in a random order each session. For each separate measurement the three observers were given a blank form to fill in the measurements results, the temperature of the water and the room temperature. It was a double-blind study design; the observers were not able to see their preceding or each other's measurements and the subjects were not able to see their own outcomes or those of the other subjects.

\section{Statistical analysis}

The raw volume data was summarized through means and standard deviations by session (three sessions) and observer (three observers). After averaging the subject's volume measurements over the three observers the volume trajectories of the ten subjects across the three sessions were depicted in a figure. In each subject the average of the nine volume measurements and the nine deviations from that average were calculated. Then those deviations from average were plotted against the average subject's volumes using all subjects. In the resulting scatterplot reference lines were drawn calculated as \pm 1.96 times the crude (unadjusted) estimate of the within-subject standard deviation of the volume measurements.

A formal statistical analysis of the data was performed using linear mixed modelling in the total dataset of 90 observations. The following fixed effects were specified in order to account for potential systematic differences in measurements between sessions: water temperature (degrees centigrade), room temperature (degrees centigrade) and session (a 3-level categorical nominal variable). Given these fixed effects, the following sources of variability (random effects) were considered for decomposing the total variance of the measurements: the subjects (1), the observers (2) and the interactions when combining these sources: observer $x$ subject (3), observer $x$ session (4) and subject $x$ session (5). The remaining error component then is the interaction subject $x$ observer $x$ session (6). These effects were supposed to respectively stand for the variance components: between-subjects (source (1)), between-observers (source (2)), within-observers (sources (3) and (4)), within-subjects (source (5)) and the error inherent in functioning of the measurement device (source (6)). Total variance is the sum of these six components. The fixed and random effects were estimated using a restricted maximum likelihood method (REML). Reliability is represented by the intra class correlation coefficient (icc), defined as the ratio of the first component (the subjects, source (1)) to the total variance. The icc is a dimensionless number between 0 and 1 , representing the similarity of replicate measurements on the same subject. Clinically it may be more useful to define a reliability measure in the same units as the measurement involved (kilogram water). To this end a reliability index is defined as 1.96 times the square root of twice the sum of the remaining components (sources (2) to (6)), assembling all within-subject random components. It represents the maximum absolute difference in kilogram 
water allowed (on the 95\% level) between two consecutive measurements of the same subject with an unchanged arm volume.

\section{Results}

The mean age of the ten subjects was 27 years and their mean Body Mass Index (BMI) was 21. Baseline characteristics are shown in Table 6.1.

Table 6.1 Baseline characteristics of the ten healthy volunteers ( 5 males and 5 females).

\begin{tabular}{lcccc}
\hline & Mean & Std. Deviation & Minimum & Maximum \\
\hline Age (years) & 26.7 & 2.7 & 24 & 32 \\
Weight $(\mathrm{kg})$ & 67.5 & 11.1 & 53 & 85 \\
Height $(\mathrm{m})$ & 1.78 & 0.06 & 1.71 & 1.86 \\
BMI $\left(\mathrm{kg} / \mathrm{m}^{2}\right)$ & 21.3 & 2.7 & 17 & 25 \\
\hline
\end{tabular}

Summary statistics of measured volumes by observer and session are shown in Table 6.2. Notably, one volunteer was not able to attend the second session. Mean volumes appeared to be systematically lower in session 3 than in sessions 1 and 2 for each observer.

Table 6.2 Measurement results with the "Inverse Water Volumetry apparatus" (IWV-apparatus).

\begin{tabular}{lcccccc}
\hline Observer & Session & Mean $\mathbf{( k g})$ & $\mathbf{N}$ & Std. Deviation & Minimum & Maximum \\
\hline \multirow{3}{*}{1} & 1 & 2.33 & 10 & 0.46 & 1.64 & 3.07 \\
& 2 & 2.31 & 9 & 0.45 & 1.66 & 2.90 \\
& 3 & 2.24 & 10 & 0.43 & 1.63 & 2.81 \\
2 & 1 & 2.31 & 10 & 0.46 & 1.64 & 2.97 \\
& 2 & 2.30 & 9 & 0.45 & 1.60 & 2.87 \\
& 3 & 2.20 & 10 & 0.48 & 1.52 & 2.85 \\
3 & 1 & 2.30 & 10 & 0.45 & 1.63 & 2.97 \\
& 2 & 2.33 & 9 & 0.45 & 1.67 & 2.96 \\
& 3 & 2.24 & 10 & 0.46 & 1.53 & 2.92 \\
\hline
\end{tabular}

Figure 6.2 shows the volume trajectories (averaged over 3 observers) of all subjects. All subjects had a lower average volume at session 3 than at session 1 . No intersections of the trajectories were seen, meaning that all subjects kept their relative position in the distribution of volumes across sessions, indicating a high reliability. 


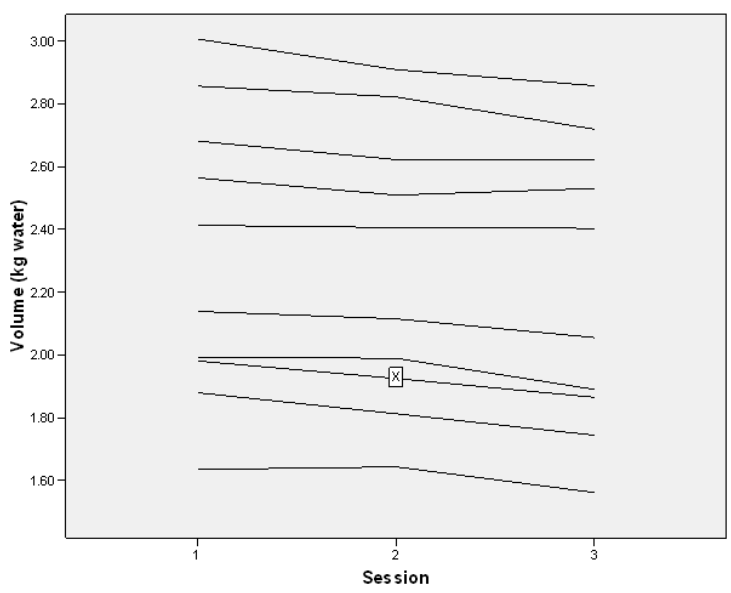

Figure 6.2 Measured volumes (averaged across three observers) by subject by session ( $\mathrm{X}=$ missing value).

Figure 6.3 shows the 9 deviations from mean volume per subject plotted against the subject's mean volume in all subjects. The vertical scatter of this plot represents the crude within-subject variability. As expected almost all points were lying between $\pm 0.125 \mathrm{~kg}$, equalling 1.96 times the crude within-subject standard deviation of $0.064 \mathrm{~kg}$. From this plot there was no suspicion of any pattern in the variability of volume measurements in relation to volume level. No adjustment was made here for fixed effects of temperature and session on volume.

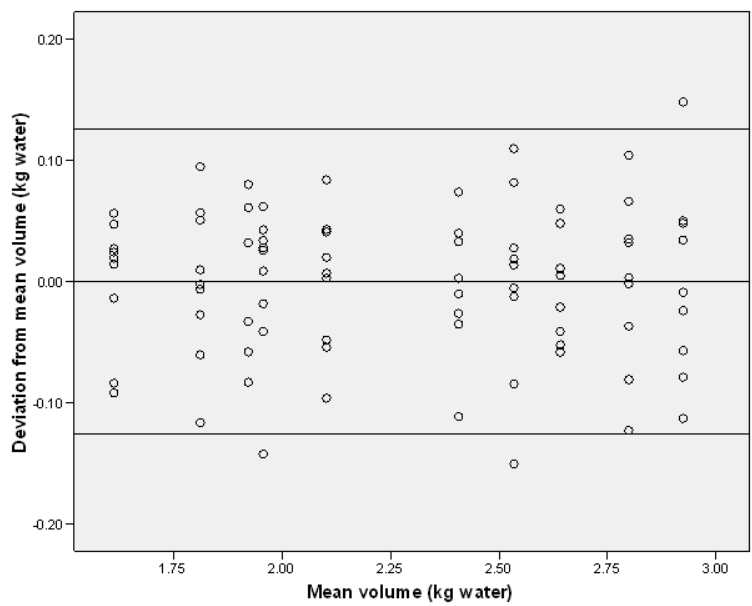

Figure 6.3 Deviation from mean volume plotted against mean volume (kg water). The upper and lower horizontal lines were drawn at a distance from the mean of 1.96 times the crude unadjusted within-subject standard deviation of 0.064 . 
The results of the linear mixed model analysis are shown in Table 6.3. Three missing observations due to one subject not attending session 2 were appropriately dealt with by using the restricted maximum likelihood method for estimating the fixed and random effects. It appeared that room temperature and water temperature did not have significant effects on the measured volumes. However, adjusted for those temperatures, the effect of the different session on measured volumes was significant $(P=0.001)$. Measurements at the third session were systematically and significantly lower than those at the preceding sessions: $0.10 \mathrm{~kg}$ lower than session 1 and $0.08 \mathrm{~kg}$ lower than session 2, corroborating the conclusions from Table 6.2 and Figure 6.2. After correction for these systematic effects, reliability of volume measurements can be judged by calculating the intra class correlation coefficient (icc) as the ratio of the subject component (1) to the total variance (7) resulting in a value of $0.99(=0.20331 /$ $0.20597)$, which is nearly perfect as already illustrated by means of Figure 6.2. The reliability index was calculated from Table 6.3 as $1.96 \times \sqrt{2} \times(0.20597-0.20331=$ $0.14 \mathrm{~kg}$, meaning that the absolute difference between two volume measurements within the same subject with an unchanged arm volume is maximally $0.14 \mathrm{~kg}$ at the 95\% level. The overall within-subject standard deviation after adjustment for the fixed

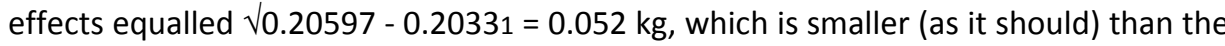
crude (unadjusted) within-subject standard deviation of $0.064 \mathrm{~kg}$ used for the reference lines in Figure 6.3.

Table 6.3 Results of a linear mixed model for analysing the reliability of the measurements with the IWV-apparatus (fixed effects in $\mathrm{kg}$; random effects in squared $\mathrm{kg}$ ).

\begin{tabular}{|c|c|c|}
\hline Variable & Fixed effects (95\% Cl) & $P$ \\
\hline Intercept & 1.677 (0.861 to 2.492$)$ & \\
\hline Water temperature $\left(+1^{\circ} \mathrm{C}\right)$ & $-0.003(-0.006$ to +0.000$)$ & 0.084 \\
\hline Room temperature $\left(+1^{\circ} \mathrm{C}\right)$ & $0.027(-0.007$ to +0.061$)$ & 0.11 \\
\hline Session & $0.099(0.063$ to 0.135$)$ & 0.001 \\
\hline 2 & $0.076(0.032$ to 0.120$)$ & 0.002 \\
\hline 3 & 0 & \\
\hline Variance component & Random effects & $\%$ \\
\hline (1) Subject & 0.20331 & 98.71 \\
\hline (2) Observer & 0.00000 & 0.00 \\
\hline (3) Observer x subject & 0.00029 & 0.14 \\
\hline (4) Observer $x$ session & 0.00001 & 0.00 \\
\hline (5) Subject x session & 0.00026 & 0.13 \\
\hline (6) Error & 0.00210 & 1.02 \\
\hline (7) Total variance & 0.20597 & 100 \\
\hline
\end{tabular}

ICC $=(1) /(7)=0.99$

The inter-observer component (2) was smaller than $0.005 \%$ of the total variance. The intra-observer components ( 3 ) and (4) added to $0.15 \%$ of total variance and the intrasubjects component (5) was $0.13 \%$ of total variance. The remaining error component 
(6) due to functioning of the measurement device was $1.02 \%$ of total variance. These sources of variation were estimated after correcting for the fixed effects mentioned in the upper part of Table 6.3.

\section{Discussion}

In this study the use of measurements with the IWV-apparatus for the measurement of repeated arm volumes can be considered a reliable and reproducible method. There was no inter-observer variability and hardly any intra-observer and intra-subject variability.

The intra class correlation coefficient defined as the ratio of the inter-subject component to the total variance reached the almost perfect value of 0.99 which is similar to the results reported in a study by Damstra et al.. ${ }^{29}$ The reliability index of the measurements with the IWV-apparatus was calculated as $0.14 \mathrm{~kg}$. This means that only absolute changes in a patient's arm volume measurement of more than $0.14 \mathrm{~kg}$ would represent a true change in arm volume, which is about $6 \%$ of the mean arm volume of $2.3 \mathrm{~kg}$. This reliability index can be considered a generalization to more than two replicate measurements of the limits of agreement as introduced by Bland and Altman ${ }^{30}$ for the comparison of two measurement methods. In Bland and Altman the limits are calculated around the fixed mean difference between the two methods. In our case the reliability index represents limits calculated around zero, in order to meet the assumption of the replicate measurements on the subject being taken under the same conditions. The linear mixed model can be considered a general tool for both types of studies.

A puzzling result was the decrease in arm volume in all subjects at session 3 compared to session 1 (Figure 6.2). Although relatively small (less than $5 \%$ of the mean volume of $2.3 \mathrm{~kg}$ ), the mean decrease of $0.10 \mathrm{~kg}$ (Table 6.3 ) was highly statistically significant because of the relatively small overall within-subject standard deviation. It is very unlikely that the arm volume of all subjects would have decreased during this experiment. Hence, we concluded that this was not a real volume effect. As we did not measure a known dummy control volume along with the other measurements we were not able to determine if this decrease could either be attributed to an artefact in the study subjects or a technical artefact of the IWV-apparatus. Although rather speculatively, we think that this decrease is the result of a learning effect in the group of subjects who in the course of the experiment learned to more carefully place their arms into the water tank.

Taking measurements with the IWV-apparatus have some limitations; it is timeconsuming (on average 15 minutes, with most time spent on the preparation), it requires many handlings with water and water tanks, it does not measure the upper 
part of the upper arm and the device is expensive. ${ }^{23,24,29}$ Other methods to measure arm volume do have limitations as well. Brorson and Höijer described (with a limited number of patients) consistent results based on the circumferential measurement method compared to water displacement methods. ${ }^{16}$ They found the circumferential measurement method useful in evaluating lymphedema because it is cheap and quick. In conclusion, the findings of this study suggest that the IWV-apparatus provides reliable and reproducible measurements so that its use for measuring arm volumes can be recommended. 


\section{References}

1. Mansel RE, Fallowfield L, Kissin M, Goyal A, Newcombe RG, Dixon JM, et al. Randomized multicenter trial of sentinel node biopsy versus standard axillary treatment in operable breast cancer: the ALMANAC Trial. J Natl Cancer Inst. 2006;98:599-609.

2. Blanchard DK, Donohue JH, Reynolds C, Grant CS. Relapse and morbidity in patients undergoing sentinel lymph node biopsy alone or with axillary dissection for breast cancer. Arch Surg. 2003;138:482-7.

3. Haid A, Koberle-Wuhrer R, Knauer M, Burtscher J, Fritzsche H, Peschina W, et al. Morbidity of breast cancer patients following complete axillary dissection or sentinel node biopsy only: a comparative evaluation. Breast Cancer Res Treat. 2002;73:31-6.

4. Leidenius M, Leivonen M, Vironen J, von Smitten K. The consequences of long-time arm morbidity in node-negative breast cancer patients with sentinel node biopsy or axillary clearance. J Surg Oncol. 2005;92:23-31.

5. Ronka R, von Smitten K, Tasmuth T, Leidenius M. One-year morbidity after sentinel node biopsy and breast surgery. Breast. 2005;14:28-36.

6. Schijven MP, Vingerhoets AJ, Rutten HJ, Nieuwenhuijzen GA, Roumen RM, van Bussel ME, et al. Comparison of morbidity between axillary lymph node dissection and sentinel node biopsy. Eur J Surg Oncol. 2003;29:341-50.

7. Schrenk P, Rieger R, Shamiyeh A, Wayand W. Morbidity following sentinel lymph node biopsy versus axillary lymph node dissection for patients with breast carcinoma. Cancer. 2000;88:608-14.

8. Swenson KK, Nissen MJ, Ceronsky C, Swenson L, Lee MW, Tuttle TM. Comparison of side effects between sentinel lymph node and axillary lymph node dissection for breast cancer. Ann Surg Oncol. 2002;9:745-53.

9. Noguchi M, Miwa K, Michigishi T, Yokoyama K, Nishijima H, Takanaka T, et al. The Role of Axillary Lymph Node Dissection in Breast Cancer Management. Breast Cancer. 1997;4:143-53.

10. Johnson KC, Kennedy AG, Henry SM. Clinical measurements of lymphedema. Lymphat Res Biol. 2014;12:216-21.

11. Herpertz U. [Measuring and documentation of edema]. Z Lymphol. 1994;18:24-30.

12. Farncombe M, Daniels G, Cross L. Lymphedema: the seemingly forgotten complication. J Pain Symptom Manage. 1994;9:269-76.

13. Voogd AC, Ververs JM, Vingerhoets AJ, Roumen RM, Coebergh JW, Crommelin MA. Lymphoedema and reduced shoulder function as indicators of quality of life after axillary lymph node dissection for invasive breast cancer. Br J Surg. 2003;90:76-81.

14. Coen JJ, Taghian AG, Kachnic LA, Assaad SI, Powell SN. Risk of lymphedema after regional nodal irradiation with breast conservation therapy. Int J Radiat Oncol Biol Phys. 2003;55:1209-15.

15. Armer JM, Stewart BR. A comparison of four diagnostic criteria for lymphedema in a post-breast cancer population. Lymphat Res Biol. 2005;3:208-17.

16. Brorson $\mathrm{H}$, Hoijer P. Standardised measurements used to order compression garments can be used to calculate arm volumes to evaluate lymphoedema treatment. J Plast Surg Hand Surg. 2012;46:410-5.

17. Bulley C, Coutts F, Blyth C, Jack W, Chetty U, Barber M, et al. A Morbidity Screening Tool for identifying fatigue, pain, upper limb dysfunction and lymphedema after breast cancer treatment: a validity study. Eur J Oncol Nurs. 2014;18:218-27.

18. Barranger E, Dubernard G, Fleurence J, Antoine M, Darai E, Uzan S. Subjective morbidity and quality of life after sentinel node biopsy and axillary lymph node dissection for breast cancer. J Surg Oncol. 2005;92:17-22.

19. Stanton AW, Badger C, Sitzia J. Non-invasive assessment of the lymphedematous limb. Lymphology. 2000;33:122-35.

20. te Slaa A, Tetteroo E, Mulder PG, Ho GH, Vos LD, Moll FL, et al. Magnetic resonance imaging reveals edema-like changes not only subcutaneously, but also in muscle tissue after femoropopliteal bypass surgery. Ann Vasc Surg. 2012;26:233-41. 


\section{Chapter 6}

21. van der Laan L, Oyen WJ, Verhofstad AA, Tan EC, ter Laak HJ, Gabreels-Festen A, et al. Soft tissue repair capacity after oxygen-derived free radical-induced damage in one hindlimb of the rat. J Surg Res. 1997;72:60-9.

22. Kettle JH, Rundle FF, Oddie TH. Measurement of upper limb volumes: a clinical method. Aus N Z J Surg. 1958;27:263-70.

23. Kaulesar Sukul DM, den Hoed PT, Johannes EJ, van Dolder R, Benda E. Direct and indirect methods for the quantification of leg volume: comparison between water displacement volumetry, the disk model method and the frustum sign model method, using the correlation coefficient and the limits of agreement. J Biomed Eng. 1993;15:477-80.

24. Megens AM, Harris SR, Kim-Sing C, McKenzie DC. Measurement of upper extremity volume in women after axillary dissection for breast cancer. Arch Phys Med Rehabil. 2001;82:1639-44.

25. Sagen A, Karesen R, Skaane P, Risberg MA. Validity for the simplified water displacement instrument to measure arm lymphedema as a result of breast cancer surgery. Arch Phys Med Rehabil. 2009; 90:803-9.

26. Oerlemans HM, Goris RJ, Oostendorp RA. Impairment level sumscore in reflex sympathetic dystrophy of one upper extremity. Arch Phys Med Rehabil. 1998;79:979-90.

27. Gjorup C, Zerahn B, Hendel HW. Assessment of volume measurement of breast cancer-related lymphedema by three methods: circumference measurement, water displacement, and dual energy X-ray absorptiometry. Lymphat Res Biol. 2010;8:111-9.

28. Klompenhouwer EG, Gobardhan PD, Beek MA, Voogd AC, Luiten EJ. The clinical relevance of axillary reverse mapping (ARM): study protocol for a randomized controlled trial. Trials. 2013;14:111.

29. Damstra RJ, Glazenburg EJ, Hop WC. Validation of the inverse water volumetry method: A new gold standard for arm volume measurements. Breast Cancer Res Treat. 2006;99:267-73.

30. Bland JM, Altman DG. Statistical methods for assessing agreement between two methods of clinical measurement. Lancet. 1986;1:307-10. 


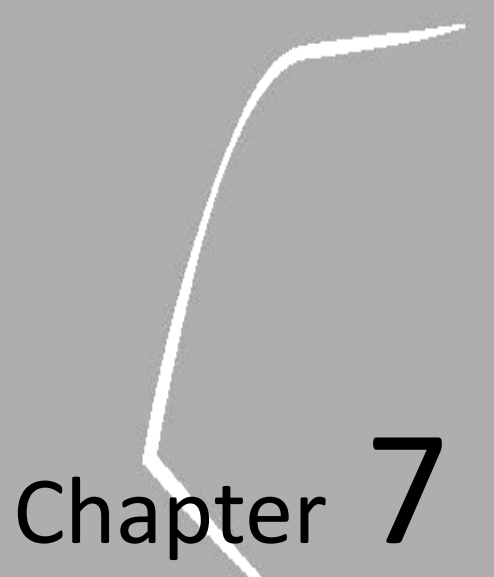

Clinical utility of Axillary Reverse Mapping (ARM) in an era of changing perceptions concerning

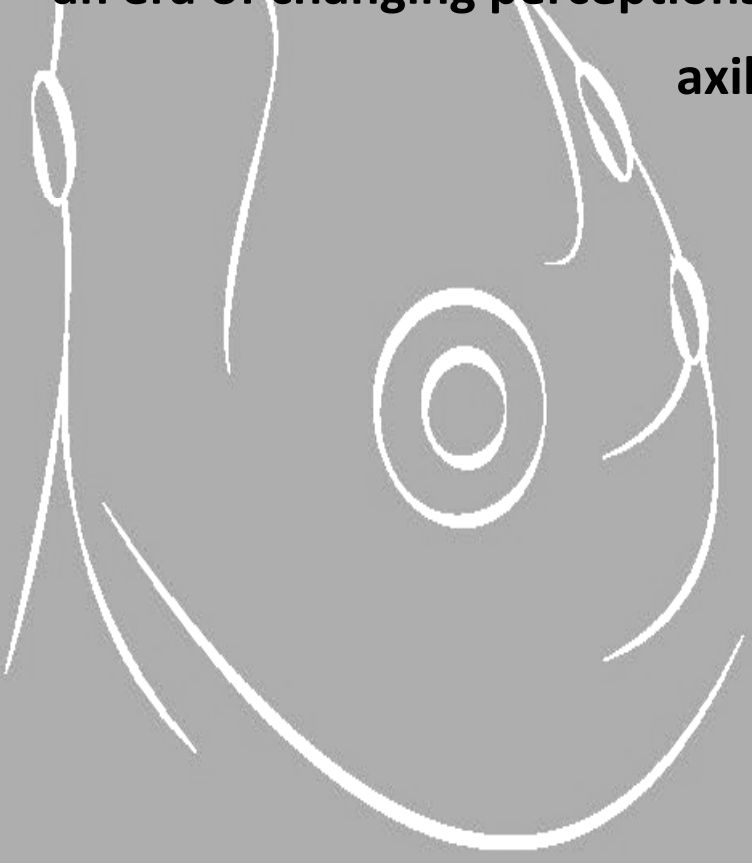

EJT Luiten, MA Beek, IT Rubio Eur J Surg Oncol. 2016;42(5):585-7 

Axillary lymph node dissection (ALND) has been a valuable technique for final staging of breast cancer patients and knowledge of the extent of axillary lymph node involvement has been important when making adjuvant treatment decisions.

The technique of ALND which was first reported in the $15^{\text {th }}$ century by the Italian surgeon Marcus Aurelius Severinus and the French surgeon Ambrose Paré and later in the $18^{\text {th }}-19^{\text {th }}$ century by Lorenz Heister, William Halsted, John Madden and others has essentially been the same over the last 150 years however refraining from Level III dissection. The surgical technique is however infamous for its accompanying morbidity, i.e. pain, numbness or paresthesia, arm and/or shoulder mobility restriction and especially upper extremity lymphedema.

Upper extremity lymphedema may occur in $7-77 \%$ in axillary surgery and is thereby the most frequent complication. ${ }^{1}$ Patients with upper extremity lymphedema are more susceptible to upper extremity wound infections, pain, restriction of arm movement all of which result in structurally lower quality of life. Consequently total health care costs are increased in patients with lymphedema.

Ever since publication of the compelling results of both American College of Surgeons Oncology Group (ACOSOG) Z0011 trial ${ }^{2}$ and the European Organisation for Research and Treatment of Cancer (EORTC) AMAROS trial ${ }^{3}$ the clinical use of completion ALND as additional axillary clearance in case of positive sentinel lymph node(s) (SLN) has been confined to second best or should even be abandoned.

Notwithstanding reports of considerable limitations, results of these trials demonstrating no difference in oncologic outcome as well as reduction of morbidity have changed our current way of thinking concerning the clinical use of completion ALND. In current practice axillary radiotherapy (ART) or even or omission of completion ALND in case of concordance with Z0011 criteria, are options which are increasingly adapted. Apart from the aforementioned ALND may still be indicated for patients with mastectomy and macrometastases in the SLN, those where more information on the number of axillary lymph nodes involved is needed or in case of residual axillary disease despite neo-adjuvant chemotherapy.

However concerns have risen that this shift towards less axillary surgery, motivated by the desire to reduce morbidity should not come at the cost of less favourable outcome. The role of the ongoing UK POSNOC (POsitive Sentinel NOde: adjuvant therapy alone versus adjuvant therapy plus Clearance or axillary radiotherapy) trial may therefore further contribute to the actual role of completion ALND in patients with macrometastatic lymph node involvement in the SLN. ${ }^{4}$

Despite the growing awareness of less invasive therapy for the additional treatment of the axilla patients presenting with involved lymph nodes as detected by ultrasound guided fine needle aspiration cytology (FNAC) at preoperative assessment should undergo axillary clearance. ${ }^{5}$

The influence of ALND on survival has always been a controversy. Despite increased rates of axillary recurrence in patients without an ALND, results of the NSABP B-04 
study did not translate into differences in survival. However the meta-analysis by Orr showed a $5 \%$ difference in overall survival. ${ }^{6}$ In the era of multimodal treatment, an increased rate of local recurrence is translated into a decreased survival, which suggests that any regional therapy may have impact in overall survival, be it either ALND or ART. 7,8

However a valuable surgical technique that has proven its value in clinical decision making for many decades is now on the verge of being discarded without efforts to refine this technique thereby striving to reach postoperative morbidity comparable to ART but excluding the specific sequelae of the latter.

ART on the other hand is also known for its possible side effects especially a lesser range of shoulder motion, when compared to a Level I-II ALND. Moreover upper extremity lymphedema, most often measured as any clinical signs or by arm circumference, may also develop following ART however to a lesser degree unless added to ALND in case of extensive nodal disease.

The basic question as to why development of upper extremity lymphedema occurs as well as surgical refinements of ALND in an effort to prevent this has not gained attention until publication of Nos et al. in 2007 and Klimberg introducing a more selective ALND introducing the addition of axillary reversed mapping (ARM). ${ }^{9,10}$ The fundamental concept of the ARM procedure is based on the findings that the upper extremity and breast each have their own discrete pathways of lymphatic drainage in the axillary basin which may sometimes interconnect. ${ }^{11}$ If upper extremity lymphedema is caused by disruption of these axillary lymphatics and/or removal of ARM lymph nodes, then being able to identify and preserve them would possibly prevent upper extremity lymphedema during SLN and/or ALND. Upper extremity lymph nodes and lymphatics in the axillary basin can be visualised by different techniques of which blue dye, which is injected subcutaneously in the medial bicipital grove of the upper part of the fore arm, is most frequently used. Generally, the blue lymph nodes are located one centimetre below the axillary vein and over or lateral to the thoracodorsal neurovascular bundle. ${ }^{12}$

Visualisation using fluorescent imaging seems to result in high visualisation rates of both ARM lymph nodes and lymphatics. However, this technique requires the use of more expensive equipment and the experience is still limited. ${ }^{13,14}$

In a non-randomised study in 60 patients to assess the incidence of breast cancerrelated lymphedema Gennaro et al. reported a statistically significantly lower risk of lymphedema of $9 \%$ in patients treated with ARM-ALND $(n=45)$ as compared to $33 \%$ in case of standard ALND ( $n=15) .{ }^{15}$ Pasko et al. used a questionnaire survey to determine the presence of lymphedema. Of the 46 surveys returned, six of the patients $(27 \%)$ in the ARM-ALND group identified themselves as having lymphedema compared with 12 of the patients (50\%) in standard-ALND group. ${ }^{16}$ Recently, Yue et al. reported results of up until now the only prospective randomized study in which preservation of the lymph drainage system of the upper limb by means of the ARM technique significantly 
reduced postoperative morbidity. In this study 265 patients, operated on by one surgeon, were included with an average follow-up period of 20 months. Upper extremity lymphedema occurred in 42 patients (33\%) in the control group versus only 7 patients $(6 \%)$ in the experimental group $(p<0.001) .{ }^{17}$ However, in this study the circumference measurement method was carried out for assessing upper extremity lymphedema which less accurate in contrast to the water-displacement method and patients treated with NAC or breast-conserving surgery were excluded. A recent meta-analysis, including 1142 patients who underwent the ARM procedure, demonstrates that the technique is feasible and low rates of upper extremity lymphedema from ARM lymphatic preservation have been reported. ${ }^{18}$

Hence, amelioration of the ALND technique thereby possibly reducing the incidence of upper extremity lymphedema to values comparable to or even better than alternative therapies may be of clinical value. Preliminary results with regard to upper extremity lymphedema and quality of life of a multicentre randomised controlled trial which is currently running in The Netherlands, analysed by volume displacement measurement, which currently is regarded as the gold standard, as well as circumference measurement and patient reported outcome measures are promising. ${ }^{19}$ Although final results will have to wait, refinements of any surgical technique should be fully appreciated before valuable surgical procedures are to be discarded, knowing that there are still patients that benefit from ALND.

Preservation of ARM lymph nodes in case of SLN-positive disease is safe since metastatic involvement is virtually absent in contrast to the other axillary lymph nodes. In case of primary clinical node positive disease administration of neo-adjuvant chemotherapy significantly lowers the rate of metastatic involvement of ARM lymph nodes. Proper selection of the aforementioned patients in whom residual metastatic involvement of ARM lymph nodes is still present despite neo-adjuvant chemotherapy allows for more aggressive axillary treatment thereby accepting higher risk of upper extremity lymphedema. ${ }^{20}$

In summary, the addition of selectively sparing upper-limb axillary lymph nodes and lymphatic drainage by means of ARM during ALND may have the potency to reduce the incidence of upper extremity lymphedema to much lesser levels as currently reported when using the classic technique. ALND remains a valuable surgical tool for breast cancer patients when indicated and refinements of the ALND technique including the ARM procedure may potentially become the standard to reduce the morbidity of the ALND without compromising the oncological outcomes in those patients that still benefit from an ALND. Further results of ongoing studies and long term follow up should be awaited for in order to appreciate the clinical value of ARMALND in the current debate concerning the different treatment options of the axilla in breast cancer patients. 


\section{References}

1. Schijven MP, Vingerhoets AJ, Rutten HJ, Nieuwenhuijzen GA, Roumen RM, van Bussel ME, et al. Comparison of morbidity between axillary lymph node dissection and sentinel node biopsy. Eur J Surg Oncol. 2003;29:341-50.

2. Giuliano AE, Hunt KK, Ballman KV, Beitsch PD, Whitworth PW, Blumencranz PW, et al. Axillary dissection vs no axillary dissection in women with invasive breast cancer and sentinel node metastasis: a randomized clinical trial. JAMA. 2011;305:569-75.

3. Donker M, van Tienhoven G, Straver ME, Meijnen P, van de Velde CJ, Mansel RE, et al. Radiotherapy or surgery of the axilla after a positive sentinel node in breast cancer (EORTC 10981-22023 AMAROS): a randomised, multicentre, open-label, phase 3 non-inferiority trial. Lancet Oncol. 2014;15:1303-10.

4. Goyal A, Dodwell D. POSNOC: A randomised trial looking at axillary treatment in women with one or two sentinel nodes with macrometastases. Clin Oncol (R Coll Radiol). 2015;27:692-5.

5. Dodwell D, Goyal A. Axillary conservation in early breast cancer. Br J Surg. 2015;102:1297-9.

6. Orr RK. The impact of prophylactic axillary node dissection on breast cancer survival--a Bayesian meta-analysis. Ann Surg Oncol. 1999;6:109-16.

7. Poortmans PM, Collette S, Kirkove C, Van Limbergen E, Budach V, Struikmans H, et al. Internal mammary and medial supraclavicular irradiation in breast cancer. N Engl J Med. 2015;373:317-27.

8. Early Breast Cancer Trialists' Collaborative G, Darby S, McGale P, Correa C, Taylor C, Arriagada R, et al. Effect of radiotherapy after breast-conserving surgery on 10-year recurrence and 15-year breast cancer death: meta-analysis of individual patient data for 10,801 women in 17 randomised trials. Lancet. 2011;378:1707-16.

9. Nos C, Lesieur B, Clough KB, Lecuru F. Blue dye injection in the arm in order to conserve the lymphatic drainage of the arm in breast cancer patients requiring an axillary dissection. Ann Surg Oncol. 2007;14:2490-6.

10. Klimberg VS. A new concept toward the prevention of lymphedema: axillary reverse mapping. J Surg Oncol. 2008;97:563-4.

11. Pavlista D, Eliska O. Analysis of direct oil contrast lymphography of upper limb lymphatics traversing the axilla -- a lesson from the past -- contribution to the concept of axillary reverse mapping. Eur J Surg Oncol. 2012;38:390-4.

12. Clough KB, Nasr R, Nos C, Vieira M, Inguenault C, Poulet B. New anatomical classification of the axilla with implications for sentinel node biopsy. Br J Surg. 2010;97:1659-65.

13. Ikeda K, Ogawa Y, Kajino C, Deguchi S, Kurihara S, Tashima T, et al. The influence of axillary reverse mapping related factors on lymphedema in breast cancer patients. Eur J Surg Oncol. 2014;40:818-23.

14. Noguchi M, Noguchi M, Nakano Y, Ohno Y, Kosaka T. Axillary reverse mapping using a fluorescence imaging system in breast cancer. J Surg Oncol. 2012;105:229-34.

15. Gennaro M, Maccauro M, Sigari C, Casalini P, Bedodi L, Conti AR, et al. Selective axillary dissection after axillary reverse mapping to prevent breast-cancer-related lymphoedema. Eur J Surg Oncol. 2013;39:1341-5.

16. Pasko JL, Garreau J, Carl A, Ansteth M, Glissmeyer M, Johnson N. Axillary reverse lymphatic mapping reduces patient perceived incidence of lymphedema after axillary dissection in breast cancer. Am J Surg. 2015;209:890-5.

17. Yue T, Zhuang D, Zhou P, Zheng L, Fan Z, Zhu J, et al. A prospective study to assess the feasibility of axillary reverse mapping and evaluate its effect on preventing lymphedema in breast cancer patients. Clin Breast Cancer. 2015;15:301-6.

18. Ahmed M, Rubio IT, Kovacs T, Klimberg VS, Douek M. Systematic review of axillary reverse mapping in breast cancer. Br J Surg. 2016;103:170-8.

19. Klompenhouwer EG, Gobardhan PD, Beek MA, Voogd AC, Luiten EJ. The clinical relevance of axillary reverse mapping (ARM): study protocol for a randomized controlled trial. Trials. 2013;14:111.

20. Beek MA, Gobardhan PD, Klompenhouwer EG, Rutten HJ, Voogd AC, Luiten EJ. Axillary reverse mapping (ARM) in clinically node positive breast cancer patients. Eur J Surg Oncol. 2015;41:59-63. 


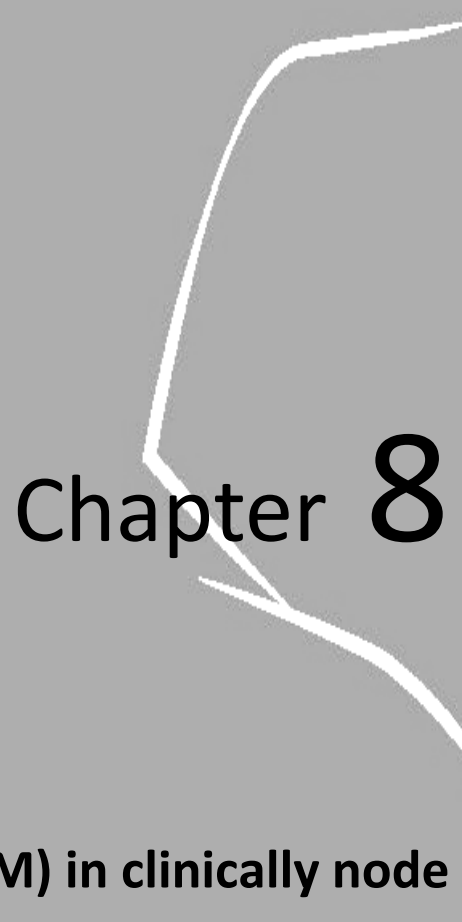

Axillary reverse mapping (ARM) in clinically node positive breast cancer patients

MA Beek, PD Gobardhan, EG Klompenhouwer, HJT Rutten, AC Voogd, EJT Luiten

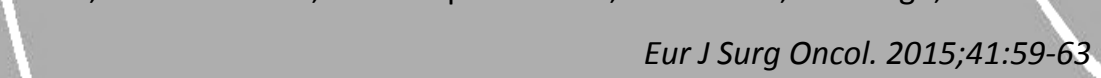




\section{Abstract}

\section{Background}

Axillary reverse mapping (ARM) is a technique to map and preserve upper-extremity lymphatic drainage during axillary lymph node dissection (ALND) in breast cancer patients.

We prospectively evaluated the metastatic involvement of ARM-nodes in patients who underwent an ALND for clinically node positive disease following (neo)adjuvant chemotherapy (NAC) in comparison to patients in whom primary ALND was performed without NAC.

\section{Patients and methods}

Patients with clinically node positive invasive breast cancer, confirmed by fine needle aspiration cytology and scheduled for primary ALND enrolled the study. Patients were separated in two groups: one group treated with NAC (NAC+ group) and one group not treated with NAC (NAC- group). ARM was performed in all patients by injecting blue dye in the ipsilateral upper extremity. During ALND ARM-nodes were first identified and removed separately, followed by a standard ALND.

\section{Results}

Ninety-one patients were included in the NAC+ and 21 patients in the NAC- group. There was no difference in the ARM visualization rate between the two groups $(86.8 \%$ for NAC+ group versus $90.5 \%$ for NAC- group, $P=0.647$ ). In the NAC+ group $16.5 \%$ of the patients had metastatic involvement of the ARM-nodes versus $36.8 \%$ of the patients in the NAC- group $(P=0.048)$.

\section{Conclusion}

The risk of metastatic involvement of ARM-nodes in clinically node positive breast cancer patients is significantly lower in patients who have received NAC. 


\section{Introduction}

Since almost 15 years, sentinel lymph node biopsy (SLNB) is standard of care in staging in cNO breast cancer patients. A negative SLNB withholds the need for a completion axillary lymph node dissection (ALND). ${ }^{1}$ Nevertheless, a primary ALND is still offered to clinically node positive patients, as proven by fine needle aspiration cytology (FNAC). ${ }^{2}$

ALND is associated with significant morbidity, i.e. postoperative lymphedema, pain, loss of sensitivity and arm/shoulder dysfunction. ${ }^{3}$ The reported incidence of lymphedema ranges from $7 \%$ and $77 \% .^{4-11}$ The incidence of lymphedema depends on whether ALND has been combined with subsequent radiotherapy but also on the definition of lymphedema used.

Most of the lymphatic drainage of the upper extremity are situated cranial to the axillary vein. Some of these lymphatics however may also be situated below the axillary vein and therefore communicate with lymph nodes which mainly drain the (female) breast. A few years ago a new technique was introduced to map the latter upper extremity lymphatics in the axilla: axillary reverse mapping (ARM). ${ }^{12-14}$ The concept of ARM is to map this part of drainage of the upper limb and thus create a road map for their preservation. If lymphedema of the upper extremity is caused by removing these lymphatics and nodes situated in the axilla, the possibility of identifying these lymphatics would enable surgeons to preserve them. In different reports the use of this technique has been described for both the SLNB as well as an ALND. ${ }^{12-23}$ In most of these reports ARM has been used for separate removal of the lymph nodes of the upper extremity. Recently, a small randomised controlled trial demonstrated a benefit (in terms of less lymphedema) for breast cancer patients in which ARM was used during an ALND to preserve upper extremity lymphatics and lymph nodes. ${ }^{24}$

A recent report from our group showed that ARM using blue dye is feasible with a visualization rate of varying between 86 and $94 \%$. Of these patients, $46 \%$ had an indication for ALND based on a positive sentinel lymph node (SLN, SLN+ group) and $53.8 \%$ underwent primary ALND because of a FNAC proven axillary metastases (CP+ group). In the first group $100 \%$ of the ARM-nodes were tumor-free compared to $22 \%$ of the patients in the second group. ${ }^{19}$

In the current report we prospectively evaluated the incidence of metastatic involvement of ARM-nodes in $\mathrm{CN}+$ patients who underwent ALND following (neo)adjuvant chemotherapy (NAC), in comparison to patients who underwent ALND without NAC. 


\section{Patients and method}

\section{Study cohort}

The study population included a consecutive serie of 112 female breast cancer patients whom received a primary ALND between October 2009 and November 2013 (Figure 8.1). The indication for a primary ALND was based on a positive FNAC of an axillary lymph node. Ethical approval for this study was provided by the local ethical commitee of the hospital. Informed consent was obtained from each participant prior to the operation.

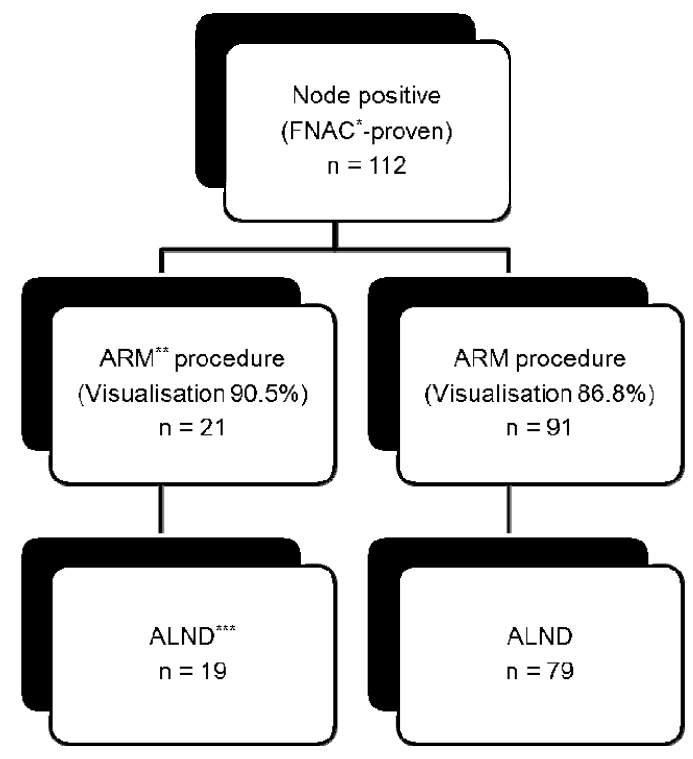

Figure 8.1 Study population of patients receiving an ALND following an ARM-procedure.

${ }^{*}$ FNAC: Fine needle aspiration cytology; ${ }^{* *}$ ARM: Axillary reverse mapping; ${ }^{* * *}$ ALND: Primary axillary lymph node dissection

\section{(Neo)adjuvant chemotherapy}

The indication for chemotherapy was determined in a multidisciplinary team including an oncologic breast surgeon, oncologist, radiotherapist and radiologist (e.g. cN+ status, large tumor size, high malignancy grade and/or young age). Treatment with NAC was discussed with the patient if the indication for chemotherapy already existed before surgery was performed 


\section{Axillary reverse mapping}

Details of the ARM-procedure have been described previously. ${ }^{19}$ In brief, blue dye (Bleu patenté V, Laboratoire Guerbet, Aulnay-sous-Bois, France) was injected in the ipsilateral upper extremity. Following a subcutaneous injection of approximately $2.0 \mathrm{ml}$ in the medial bicipital sulcus between $\mathrm{m}$. biceps brachii and $\mathrm{m}$. triceps brachii, the injection site was massaged gently for 5 minutes.

\section{Pathological examination}

Primary tumor diameter, malignancy grade applying the modified Bloom and Richardson (BR) grade, $^{25}$ estrogen (ER) and progesteron (PR) -receptorstatus and HER2/neu status were assessed. ARM-nodes were analyzed separately. In accordance with the national pathology protocol (26) all retrieved axillary lymph nodes (including the ARM-nodes) were embedded in paraffin and two central cuts from every lymph node were examined after staining with hematoxylin and eosine (H\&E) and immunohistochemically (IHC) with an antibody against keratin.

\section{Classification of patients}

Patients were classified into two groups. The first group consisted of patients treated with neoadjuvant chemotherapy (NAC+ group) and the second group consisted of patients treated with a primary ALND and adjuvant chemotherapy (NAC- group). Intraoperative visualization/identification rates as well as the proportion of ARM and axillary nodes with metastatically involved were evaluated for both groups. The number of additionally involved lymph nodes was defined as the overall number of metastatic nodes minus the one node that was proven to contain metastases (by FNAC).

\section{Statistical analysis}

Statistical analyses were performed using SPSS, version 21.0 (SPSS, Inc., Chicago, USA). Chi-square analysis was performed to evaluate proportional differences between the two groups regarding the proportion of patients with metastatic lymph node involvement. $P$ values less than 0.05 were considered significant.

\section{Results}

The mean age of the 112 patients was 55.5 years. The NAC+ group consisted of 91 patients and the NAC- group of 21 patients. Baseline characteristics for both 
groups are shown in Table 8.1. Notably, woman in the NAC+ group were younger and had larger tumors.

The overall identification rate of ARM-nodes by means of the blue dye technique was $87.5 \%$. There were no differences in the visualization rate between the two groups (86.8\% for NAC+ group and $90.5 \%$ for NAC- group, $P=0.647$, Table 8.1 ).

Table 8.1 Baseline characteristics for 112 breast cancer patients, according to use of neo-adjuvant chemotherapy (NAC).

\begin{tabular}{|c|c|c|c|}
\hline Characteristic & $\begin{array}{l}\text { NAC+ } \\
N=91\end{array}$ & $\begin{array}{l}\text { NAC- } \\
N=21\end{array}$ & $P$ \\
\hline \multicolumn{4}{|l|}{ Age, years } \\
\hline Mean (Range) & $53.4 \pm 10.0$ & $64.6 \pm 11.8$ & 0.000 \\
\hline \multicolumn{4}{|l|}{ Tumor size, $\mathrm{cm}^{1,2}$} \\
\hline Mean (Range) & $4.6(4.1-5.1)$ & $3.3(2.6-4.0)$ & 0.007 \\
\hline \multicolumn{4}{|l|}{ Tumor size $(\%)^{2}$} \\
\hline$<1 \mathrm{~cm}$ & $44(48.4)$ & $1(4.8)$ & 0.001 \\
\hline $1-2 \mathrm{~cm}$ & $11(12.1)$ & $8(38.1)$ & \\
\hline $2-3 \mathrm{~cm}$ & $13(14.3)$ & $4(19.0)$ & \\
\hline$>3 \mathrm{~cm}$ & $23(25.3)$ & $8(38.1)$ & \\
\hline \multicolumn{4}{|l|}{ Bloom-Richardson grade (\%) } \\
\hline Well differentiated & $12(13.2)$ & $2(9.5)$ & 0.004 \\
\hline Moderately differentiated & $27(29.7)$ & $10(47.6)$ & \\
\hline Poorly differentiated & $18(19.8)$ & 9 (42.9) & \\
\hline Unknown ${ }^{3}$ & $34(37.4)$ & $0(0.0)$ & \\
\hline Estrogen receptor positive (\%) & $59(64.8)$ & $17(81.0)$ & 0.154 \\
\hline Progesterone receptor positive (\%) & $44(48.4)$ & $12(57.1)$ & 0.705 \\
\hline Her2Neu receptor positive (\%) & $30(33.0)$ & $3(14.3)$ & 0.091 \\
\hline Visualisation ARM node(s) (\%) & $79(86.8)$ & $19(90.5)$ & 0.647 \\
\hline
\end{tabular}

No significant difference was found concerning the mean number of lymph nodes removed per patients during the axillary procedure including the ARM lymph nodes between the two groups (16.96 for NAC+ group and 18.42 for NAC- group, $P=0.485$, Table 8.2).

The risk of axillary lymph node involvement of both groups is shown in Table 8.2. No difference was found in the number of patients with axillary lymph node metastases: 57 out of 79 patients $(72.2 \%)$ in the NAC+ group compared to 17 out of 19 patients (89.5\%) in the NAC- group $(P=0.115)$. Not surprisingly, NAC was associated with a lower risk of mean number of positive lymph nodes with ALND (2.44 vs. 5.11, $P=0.002$ ).

Metastatic involvement of the ARM-nodes was found in 13 of 79 patients (16.5\%) in the NAC+ group compared to 7 of 19 patients $(36.8 \%)$ in the NAC-group $(P=0.048)$. 
The incidence of ARM node involvement among the patients with axillary lymph node metastases was $22.8 \%(13 / 57)$ in the NAC+ group and $41.1 \%(7 / 17)$ in the NAC-group $(P=0.158)$.

Table 8.2 Number of axillary and ARM nodes removed, number of positive axillary and ARM nodes and number and percentage of patients with axillary and ARM metastases, according to use of neo-adjuvant chemotherapy (NAC).

\begin{tabular}{|c|c|c|c|}
\hline Characteristic & $\begin{array}{l}\text { NAC+ } \\
N=79\end{array}$ & $\begin{array}{l}\text { NAC- } \\
N=19\end{array}$ & $P$ \\
\hline Axillary metastases & $57(72 \%)$ & $17(90 \%)$ & $0.115^{\#}$ \\
\hline Axillary metastases without ARM metastases & $44(56 \%)$ & $10(53 \%)$ & $0.377^{\#}$ \\
\hline Axillary metastases with ARM metastases & $13(17 \%)$ & $7(37 \%)$ & $0.048^{\#}$ \\
\hline ARM metastases without axillary metastases & 0 & 0 & \\
\hline Patients without ARM and axillary metastases & $22(28 \%)$ & $2(11 \%)$ & $0.115^{\#}$ \\
\hline \multicolumn{4}{|c|}{ Total number of lymph nodes removed, including ARM nodes } \\
\hline Mean (Range) & $16.96(4-30)$ & $18.42(10-38)$ & $0.485^{*}$ \\
\hline \multicolumn{4}{|l|}{ Total number of ARM lymph nodes removed } \\
\hline Mean (Range) & $1.66(1-9)$ & $1.58(1-4)$ & $0.678^{*}$ \\
\hline \multicolumn{4}{|c|}{ Total number of positive lymph nodes, including ARM nodes } \\
\hline Mean (Range) & $2.44(0-18)$ & $5.11(0-15)$ & $0.002^{*}$ \\
\hline \multicolumn{4}{|l|}{ Total number of positive ARM lymph nodes } \\
\hline Mean (Range) & $0.20(0-2)$ & $0.32(0-1)$ & $0.172^{\#}$ \\
\hline
\end{tabular}

* Mann-Whitney U test; ${ }^{\#}$ Chi-square test.

\section{Discussion}

This report on the incidence of metastasis in ARM nodes, demonstrates that the metastatic burden is reduced in patients when treated with NAC. As expected, NAC also decreased the percentage of additional positive axillary lymph nodes. According to other reports on ARM, ARM-nodes in both groups were less likely to show tumor involvement than the other axillary lymph nodes. ${ }^{12,13,17,19,20,22,23}$

The concept underlying the ARM procedure assumes that the lymphatic pathway from the upper extremity is not involved in the metastatic spread from the primary breast tumor. ${ }^{13,14,20,27}$ However, in recent feasibility studies $14 \%$ to $43 \%$ of the patients were reported to have metastatic tumor deposits in the ARM nodes. ${ }^{12,13,17,20-22,28}$ These studies did not focus on patients with clinically node positive breast cancer and also did not make a distinction between patients receiving NAC and those treated with a primary ALND.

A completion ALND for patients with a positive SLN is under debate. However, an ALND is still the standard treatment for $\mathrm{cN}+$ patients. ${ }^{2}$ Performing an ALND in these patients exposes them to a substantial risk of developing lymphedema of the upper 
extremity, especially when combined with radiotherapy. The risk of $16.5 \%$ of having positive ARM-nodes, found in this study in $\mathrm{cN}+$ patients receiving NAC, is lower than the $27 \%$ from the 20011 trial. $^{29}$ On the other hand, a direct comparison between these groups is difficult because most patients from the $Z 0011$ trial received adjuvant systemic therapy. Preserving ARM nodes and lymphatic drainage in these patients may reduce the incidence of lymphedema after ALND. Currently, a study is performed in the Netherlands; this multicentre trial randomizes SLN+ patients between a standard ALND and an ARM-ALND. Probably this trial will provide evidence whether axillary lymph node preservation by means of ARM can decrease the side-effects of an ALND. $^{30}$

In the present study, the high visualization rate of $86.8 \%-90.5 \%$ by using blue dye only, is similar to the results in our previous study. ${ }^{19}$ The visualization rate compares favourably to visualization rates reported by others, which vary between $50 \%$ and $89 \%$. ${ }^{13,17,20,22,23}$ Compared to radioisotopes or fluorescent dyes, using blue dye for ARM is advantageous since it is easy to perform and inexpensive.

In conclusion, in patients with clinically node positive breast cancer the incidence of metastases in the ARM-nodes was significantly lower for those in whom ALND was preceded by NAC, as compared to those without NAC. Further studies are needed to provide sufficient evidence that these patients are suitable candidates for the ARM procedure with selective preservation of these specific nodes. 


\section{References}

1. Maaskant AJ, van de Poll-Franse LV, Voogd AC, Coebergh JW, Tutein Nolthenius-Puylaert MC, Nieuwenhuijzen GA. Stage migration due to introduction of the sentinel node procedure: a population-based study. Breast Cancer Res Treat. 2009;113:173-9.

2. Tausch C, Baege A, Dietrich D, Vergin I, Heuer $\mathrm{H}$, Heusler $\mathrm{RH}$, et al. Can axillary reverse mapping avoid lymphedema in node positive breast cancer patients? Eur J Surg Oncol. 2013;39:880-6.

3. Noguchi M, Miwa K, Michigishi T, Yokoyama K, Nishijima H, Takanaka T, et al. The Role of Axillary Lymph Node Dissection in Breast Cancer Management. Breast Cancer. 1997;4:143-53.

4. Mansel RE, Fallowfield L, Kissin M, Goyal A, Newcombe RG, Dixon JM, et al. Randomized multicenter trial of sentinel node biopsy versus standard axillary treatment in operable breast cancer: the ALMANAC Trial. J Natl Cancer Inst. 2006;98:599-609.

5. Blanchard DK, Donohue JH, Reynolds C, Grant CS. Relapse and morbidity in patients undergoing sentinel lymph node biopsy alone or with axillary dissection for breast cancer. Arch Surg. 2003;138:482-7.

6. Haid A, Koberle-Wuhrer R, Knauer M, Burtscher J, Fritzsche H, Peschina W, et al. Morbidity of breast cancer patients following complete axillary dissection or sentinel node biopsy only: a comparative evaluation. Breast Cancer Res Treat. 2002;73:31-6.

7. Leidenius M, Leivonen M, Vironen J, von Smitten K. The consequences of long-time arm morbidity in node-negative breast cancer patients with sentinel node biopsy or axillary clearance. J Surg Oncol. 2005;92:23-31.

8. Ronka R, von Smitten K, Tasmuth T, Leidenius M. One-year morbidity after sentinel node biopsy and breast surgery. Breast. 2005;14:28-36.

9. Schijven MP, Vingerhoets AJ, Rutten HJ, Nieuwenhuijzen GA, Roumen RM, van Bussel ME, et al. Comparison of morbidity between axillary lymph node dissection and sentinel node biopsy. Eur J Surg Oncol. 2003;29:341-50.

10. Schrenk P, Rieger R, Shamiyeh A, Wayand W. Morbidity following sentinel lymph node biopsy versus axillary lymph node dissection for patients with breast carcinoma. Cancer. 2000;88:608-14

11. Swenson KK, Nissen MJ, Ceronsky C, Swenson L, Lee MW, Tuttle TM. Comparison of side effects between sentinel lymph node and axillary lymph node dissection for breast cancer. Ann Surg Oncol. 2002;9:745-53.

12. Nos C, Kaufmann G, Clough KB, Collignon MA, Zerbib E, Cusumano $P$, et al. Combined axillary reverse mapping (ARM) technique for breast cancer patients requiring axillary dissection. Ann Surg Oncol. 2008;15:2550-5.

13. Thompson M, Korourian S, Henry-Tillman R, Adkins L, Mumford S, Westbrook KC, et al. Axillary reverse mapping (ARM): a new concept to identify and enhance lymphatic preservation. Ann Surg Oncol. 2007;14:1890-5.

14. Klimberg VS. A new concept toward the prevention of lymphedema: axillary reverse mapping. J Surg Oncol. 2008;97:563-4.

15. Ponzone R, Cont NT, Maggiorotto F, Cassina E, Mininanni P, Biglia N, et al. Extensive nodal disease may impair axillary reverse mapping in patients with breast cancer. J Clin Oncol. 2009;27:5547-51.

16. Boneti C, Korourian S, Diaz Z, Santiago C, Mumford S, Adkins L, et al. Scientific Impact Award: Axillary reverse mapping (ARM) to identify and protect lymphatics draining the arm during axillary lymphadenectomy. Am J Surg. 2009;198:482-7.

17. Casabona F, Bogliolo S, Valenzano Menada M, Sala P, Villa G, Ferrero S. Feasibility of axillary reverse mapping during sentinel lymph node biopsy in breast cancer patients. Ann Surg Oncol. 2009;16: 2459-63.

18. Deng H, Chen L, Jia W, Chen K, Zeng Y, Rao N, et al. Safety study of axillary reverse mapping in the surgical treatment for breast cancer patients. J Cancer Res Clin Oncol. 2011;137:1869-74.

19. Gobardhan PD, Wijsman JH, van Dalen T, Klompenhouwer EG, van der Schelling GP, Los J, et al. ARM: axillary reverse mapping - the need for selection of patients. Eur J Surg Oncol. 2012;38:657-61. 


\section{Chapter 8}

20. Boneti C, Korourian S, Bland K, Cox K, Adkins LL, Henry-Tillman RS, et al. Axillary reverse mapping: mapping and preserving arm lymphatics may be important in preventing lymphedema during sentinel lymph node biopsy. J Am Coll Surg. 2008;206:1038-42.

21. Noguchi M, Yokoi M, Nakano Y. Axillary reverse mapping with indocyanine fluorescence imaging in patients with breast cancer. J Surg Oncol. 2010;101:217-21.

22. Nos C, Lesieur B, Clough KB, Lecuru F. Blue dye injection in the arm in order to conserve the lymphatic drainage of the arm in breast cancer patients requiring an axillary dissection. Ann Surg Oncol. 2007; 14:2490-6.

23. Ponzone R, Mininanni P, Cassina E, Sismondi P. Axillary reverse mapping in breast cancer: can we spare what we find? Ann Surg Oncol. 2008;15:390-1.

24. Boneti C, Badgwell B, Robertson Y, Korourian S, Adkins L, Klimberg V. Axillary reverse mapping (ARM): initial results of phase II trial in preventing lymphedema after lymphadenectomy. Minerva Ginecol. 2012;64:421-30.

25. Elston CW, Ellis IO. Pathological prognostic factors in breast cancer. I. The value of histological grade in breast cancer: experience from a large study with long-term follow-up. Histopathology. 1991; 19:403-10.

26. NOABON. Landelijke richtlijn mammacarcinoom 2.0. wwwoncolinenl/mammacarcinoom.

27. Casabona F, Bogliolo S, Ferrero S, Boccardo F, Campisi C. Axillary reverse mapping in breast cancer: a new microsurgical lymphatic-venous procedure in the prevention of arm lymphedema. Ann Surg Oncol. 2008;15:3318-9.

28. Bedrosian I, Babiera GV, Mittendorf EA, Kuerer HM, Pantoja L, Hunt KK, et al. A phase I study to assess the feasibility and oncologic safety of axillary reverse mapping in breast cancer patients. Cancer. 2010;116:2543-8.

29. Giuliano AE, Hunt KK, Ballman KV, Beitsch PD, Whitworth PW, Blumencranz PW, et al. Axillary dissection vs no axillary dissection in women with invasive breast cancer and sentinel node metastasis: a randomized clinical trial. JAMA. 2011;305:569-75.

30. Klompenhouwer EG, Gobardhan PD, Beek MA, Voogd AC, Luiten EJ. The clinical relevance of axillary reverse mapping (ARM): study protocol for a randomized controlled trial. Trials. 2013;14:111. 

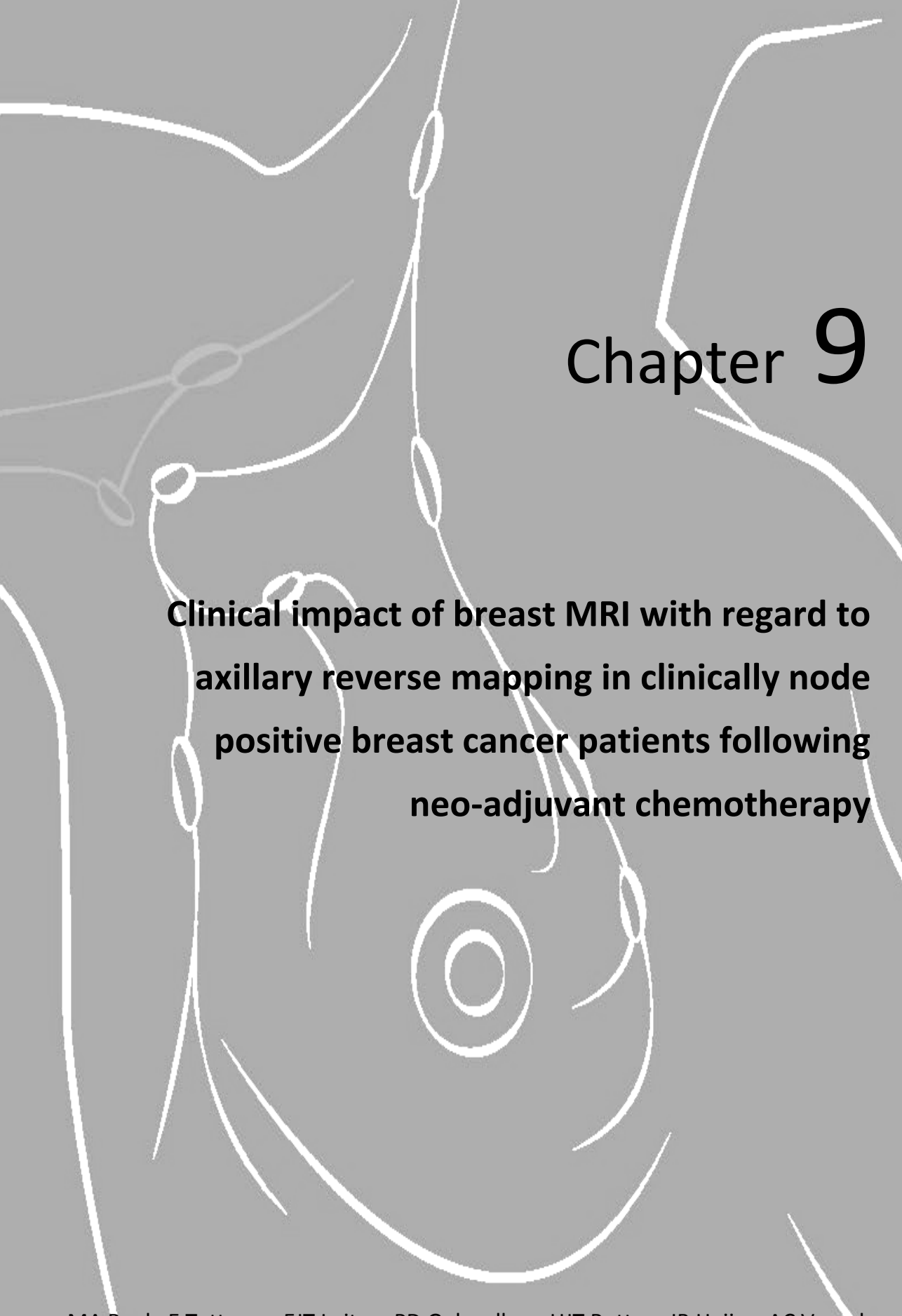

MA Beek, E Tetteroo, EJT Luiten, PD Gobardhan, HJT Rutten, JB Heijns, AC Voogd, EG Klompenhouwer Eur J Surg Oncol. 2016;42(5):672-8 


\section{Abstract}

\section{Background}

Axillary reverse mapping (ARM) is a technique that discerns axillary lymphatic drainage of the arm from the breast. In the current study we retrospectively evaluated the incidence of metastatic axillary lymph node involvement, including ARM lymph nodes, in clinically node positive breast cancer patients (cN+ patients) in whom neo-adjuvant chemotherapy (NAC) was administered followed by primary ALND using breast MRI.

\section{Patients and methods}

Data from $98 \mathrm{cN}+$ breast cancer patients were analysed retrospectively. Patients without residual axillary disease at breast MRI following NAC (RAD-, $n=64$ ) were compared with patients with residual axillary disease ( $R A D+, n=34)$. Presence of suspect axillary lymph nodes on pre-NAC and post-NAC breast MRI was determined by experienced breast radiologists and was correlated to histopathological findings.

\section{Results}

In the RAD- group residual axillary disease on pathological analysis following NAC was found in 25 patients (39.1\%), as compared to 24 patients $(70.6 \%)$ in the RAD+ group $(P=0.003)$. Metastatic involvement of ARM lymph nodes following NAC was demonstrated in 5 patients $(7.8 \%)$ in the RAD- group as compared to 10 patients (29.4\%) in the RAD+ group $(P=0.005)$.

\section{Conclusion}

Breast MRI following NAC is not suitable to detect residual metastatic disease of the axilla. However, breast MRI post-NAC may be of use to identify $\mathrm{cN}+$ patients with a low risk of ARM lymph node metastases. This may help to select a subgroup of $\mathrm{cN}+$ patients in whom sparing of ARM lymph nodes during axillary lymph node dissection can be considered. 


\section{Introduction}

Axillary lymph node dissection (ALND) in breast cancer patients has been performed for disease control, tumour staging and decision-making regarding the use of adjuvant systemic treatment and locoregional (axillary) radiotherapy. ${ }^{1,2}$ During the past twenty years, the treatment and staging of the axilla has changed rapidly. Nowadays, primary ALND is still standard of care in clinically node positive patients, as proven by ultrasound (US) guided fine needle aspiration cytology (cN+ patients). ${ }^{3,4}$ However, these patients are at risk for complications such as upper extremity lymphedema, arm and/or shoulder dysfunction and paresthesia.,

In 2007, axillary reverse mapping (ARM) was introduced as a technique to identify upper extremity lymphatics in the axilla. ${ }^{7-10}$ This procedure aims to preserve lymph nodes and/or lymphatic drainage from the arm during ALND, thereby reducing the risk of post-operative upper extremity lymphedema. Currently, a multicentre study is carried out in the Netherlands randomizing sentinel node positive patients between a standard ALND and an ARM-ALND. ${ }^{11}$

We have recently reported that saving upper extremity lymph nodes and lymphatics using the ARM procedure is feasible and oncologically safe for patients with positive sentinel lymph nodes. ${ }^{12}$ A significantly lower risk of metastases in ARM lymph nodes was demonstrated in patients with $\mathrm{cN}+$ patients receiving neo-adjuvant chemotherapy (NAC) followed by primary ALND, as compared to those without NAC. ${ }^{13}$ Our study and studies by Deng et al. and Noguchi et al. showed that metastatic involvement of the ARM lymph nodes does not occur if the other axillary lymph nodes do not contain metastatic disease. ${ }^{14,15}$

Several studies have shown that breast magnetic resonance imaging (MRI) can detect axillary node metastases in patients whom NAC was administered. ${ }^{16-19}$ Moreover, Javid et al. suggested that breast MRI following NAC might be of use in predicting the presence of residual nodal disease. They reported a sensitivity of $85.7 \%$ and a specificity of $89 \%$ for the detection of residual pathologic axillary lymph node disease. ${ }^{17}$ In the current Dutch medical practice, a breast MRI following NAC is recommended for all breast cancer patients in order to monitor disease response.

In this study, we retrospectively evaluated the incidence of metastatic axillary lymph node involvement including ARM lymph nodes in $\mathrm{cN}+$ patients who underwent primary ALND, based on both pre- and post-NAC breast MRI findings. 


\section{Patients and method}

\section{Patient selection}

We included 98 consecutive $\mathrm{cN}+$ breast cancer patients from our prospective breast cancer database at the Breast Cancer Department of Amphia Hospital Breda, The Netherlands. This database contains 234 breast cancer patients in whom the ARM procedure was carried out since October 2009. All patients in whom primary ALND were carried out, including a successful ARM lymph node visualisation, following NAC were analysed (Figure 9.1). The indication for primary ALND was based on a cytology proven positive axillary lymph node. In all patients a baseline- and a final MRI were carried out. Medical ethical approval for this study was provided by the local medical ethical committee of the hospital. Informed consent was obtained from each participant prior to the operation.

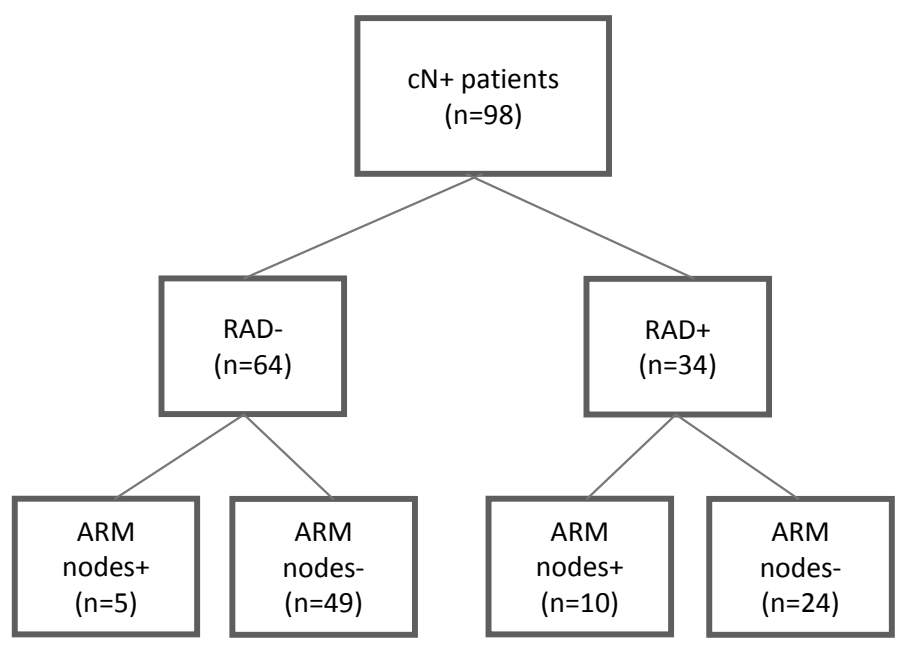

Figure 9.1 Study population of $\mathrm{CN}+$ patients receiving a primary ALND following NAC.

\section{Neo-adjuvant chemotherapy}

The selection of patients for NAC was carried out by a multidisciplinary team, consisting of an oncologic breast surgeon, medical oncologist, radiotherapist, pathologist and radiologist. In this study indication for NAC was based on histologically proven $\mathrm{cN}+$ status. NAC consisted in all Her2-neu negative cases of a third generation scheme: either six cycles of Docetaxel $75 \mathrm{mg} / \mathrm{m}^{2}$, Adriamycin $50 \mathrm{mg} / \mathrm{m}^{2}$ and Cyclophosphamide $500 \mathrm{mg} / \mathrm{m}^{2}$ intravenously every three weeks (TAC), or three cycles 
of FEC (Fluorouracil $600 \mathrm{mg} / \mathrm{m}^{2}$ intravenously, Epirubicin $90 \mathrm{mg} / \mathrm{m}^{2}$ intravenously, and Cyclophosphamide $600 \mathrm{mg} / \mathrm{m}^{2}$ intravenously every 3 weeks) followed by three cycles of Docetaxel (100 mg/m $\mathrm{m}^{2}$ intravenously every 3 weeks). In patients with Her2-neu amplified tumours (Her2-neu positive), the scheme predominantly used was four courses of AC (Adriamycin $60 \mathrm{mg} / \mathrm{m}^{2}$ and Cyclophosphamide $600 \mathrm{mg} / \mathrm{m}^{2}$ intravenously every 3 weeks), followed by 12 weekly courses of Paclitaxel $80 \mathrm{mg} / \mathrm{m}^{2}$ and Trastuzumab.

\section{Axillary reverse mapping}

The technique of the ARM procedure has been described previously. ${ }^{11-13}$ In summary, to map the axillary lymph node drainage of the upper extremity, approximately 1.0$2.0 \mathrm{ml}$ methylene blue dye (Bleu patenté $\mathrm{V}$, Laboratoire Guerbet, Aulnay-sous-Bois, France) was injected subcutaneously in the ipsilateral upper extremity at the medial inter-muscular groove between biceps muscle and triceps muscle. Subsequently, the injection site was massaged gently for 5 minutes.

\section{MRI protocol}

A baseline contrast-enhanced dynamic breast MRI was performed in all patients preceding NAC. To evaluate residual disease, a final MRI was performed after completing NAC.

All breast MR examinations were performed in a prone position using a dedicated breast coil. From November 2009 to July 2012, in 61 patients a 1.5T unit was used (MAGNETOM Avanto; Siemens Healthcare, Germany) and from August 2012 to December 2014, in 37 patients a 3.0T unit was used (MAGNETOM Skyra, Siemens Healthcare, Germany). The imaging protocol included the following sequences: (1) an axial T2-weighted turbo inversion recovery magnitude (TIRM) sequence (for 1.5T: 34 images, slice thickness $4 \mathrm{~mm}$; for $3 \mathrm{~T}$ : 48 images, slice thickness $3 \mathrm{~mm}$ ); (2) an unenhanced fast low angle shot (FLASH) three-dimensional (3D) T1-weighted gradient echo sequence without fat-suppression (for 1.5T: 160 images; for 3T: 176 images; slice thickness $1 \mathrm{~mm}$ ); (3) an axial FLASH-3D T1-weighted dynamic contrast-enhanced gradient echo sequence with fat-suppression and subtraction (for 1.5T: 160 images; for 3T: 176 images; slice thickness $0,9 \mathrm{~mm}$ ), acquired before and 5 times after i.v. injection of $30 \mathrm{ml}$ Gadoterate Meglumine (Gd-DOTA, DOTAREM, Guerbet, France). Post-contrast scanning was started 60 seconds after the start of contrast medium administration (total acquisition time for the 6 measurements $6: 42 \mathrm{~s}$ ); (4) an axial delayed contrast-enhanced FLASH-3D T1-weighted gradient echo sequence with fatsuppression, acquired following the dynamic scan (for 1.5T: 160 images; for 3T: 240 images; slice thickness of $0.7 \mathrm{~mm}$ ). 


\section{Image analysis}

In our institution, all standard of care dynamic contrast material-enhanced MRI breast examinations were independently analyzed by one of three breast radiologists, all with at least 20 years of experience in conventional breast imaging and at least 7 years of experience in breast MRI. In the present study, one of these radiologists analyzed the MR images from the included patients for a second time. This radiologist was blinded to the reported MR findings and histopathological outcomes. In case of discrepancy between the second reader and the original radiology report, consensus image interpretation was performed with one of the two other breast radiologists. Both were blinded for final histopathological outcome. A standard PACS-workstation was used for analysis (IMPAX, AGFA, Belgium).

The morphology of the axillary lymph nodes was primarily evaluated on axial T1-weighted images obtained without fat suppression. ${ }^{16}$ A lymph node was considered suspicious for metastasis when it had at least one of the following characteristics: (a) a round shape on 2 contiguous images and a diameter larger than $5 \mathrm{~mm}$; (b) cortical thickness of $3 \mathrm{~mm}$ or more; (c) absence of a fatty hilum. The total number of suspicious lymph nodes was recorded for every patient.

No residual axillary disease of the axilla after NAC was defined as the absence of any suspicious lymph nodes determined by one of the aforementioned characteristics.

\section{Histopathological analysis}

Primary tumour diameter, the modified Bloom and Richardson (BR) grade, ${ }^{20}$ estrogen receptor (ER) status, progesterone receptor (PR) status and Her2-neu status were assessed. ARM lymph nodes were removed and analysed separately from the other axillary lymph nodes. In accordance with the national pathology protocol $^{21}$ all retrieved axillary lymph nodes (including the ARM lymph nodes) were embedded in paraffin and two central cuts from every lymph node were examined after staining with hematoxylin and eosine (H\&E) according to the Dutch Guidelines.

\section{Classification of patients}

Patients were subdivided into two groups. The first group consisted of patients without residual axillary disease on the final breast MRI after NAC (RAD- group). Patients without radiological signs of axillary lymph node disease on the baseline MRI as well as on the final MRI after NAC were also included in the RAD- group. The second group consisted of patients with MRI findings suggesting residual axillary disease on the final MRI after NAC (RAD+ group). This group consisted of patients with a partial response, stable disease or with progressive disease on the final MRI.

The incidence of both ARM and axillary lymph nodes with metastatic involvement were evaluated for the two groups. The number of additionally involved lymph nodes 
was defined as the overall number of metastatic nodes minus the one single node that was already initially proven to contain metastases by FNAC.

\section{Statistical analysis}

Statistical analyses were performed using SPSS, version 21.0 (SPSS, Inc., Chicago, USA). Chi-square analysis was performed to evaluate differences between the two groups regarding the proportion of patients with metastatic lymph node involvement. $P$ values less than 0.05 were considered statistically significant. The positive predictive value and negative predictive value and corresponding $95 \%$ confidence intervals $(95 \% \mathrm{Cl})$ of MRI were calculated for the detection of axillary lymph nodes in general and ARM lymph nodes.

\section{Results}

Between November 2009 and December 2014, 98 cN+ patients underwent primary ALND following NAC, including a successfully performed ARM procedure, in our institution. The RAD- group consisted of 64 patients and the RAD+ group of 34 patients.

Baseline characteristics of both groups were largely comparable (Table 9.1), except for the Her2neu status, which was significantly more likely to be positive in the RADgroup ( 27 patients (42\%) versus 4 patients (12\%); $P=0.006)$. Furthermore patients in the RAD+ group had larger tumours at baseline when compared to the RAD- group $(P=0.033$, Table 9.1).

\section{MRI data}

The initial number of suspect lymph nodes found on MRI pre-NAC were lower in the RAD- group when compared to the RAD+ group (mean 1.9 versus 2.6; $P=0.018$, Table 9.2).

In seven patients in the RAD- group no suspicious axillary lymph nodes were demonstrated on both pre-NAC and post-NAC MRI. Presence of $\mathrm{CN}+$ in these seven patients was already proven by US findings and subsequent FNAC of a suspicious axillary lymph node. In the RAD+ group, 29 patients (85\%) showed a partial response, four patients (12\%) showed no response and one patient (3\%) showed progressive disease in the axilla after NAC (Table 9.2). 
Table 9.1 Baseline characteristics for 98 breast cancer patients, with and without residual axillary disease at breast MRI following NAC.

\begin{tabular}{|c|c|c|c|}
\hline Characteristic & $\begin{array}{c}\text { RAD- } \\
(n=64)\end{array}$ & $\begin{array}{c}\text { RAD+ } \\
(n=34)\end{array}$ & $P$ \\
\hline \multicolumn{4}{|l|}{ Age, years } \\
\hline Mean (Range) & $52(33-74)$ & $55(36-68)$ & $0.148^{b}$ \\
\hline \multicolumn{4}{|l|}{ Histology (\%) } \\
\hline Ductal & $49(76.6)$ & $31(91.2)$ & \\
\hline Lobular & $5(7.8)$ & 0 & \\
\hline Other & $8(12.6)$ & $2(5.8)$ & \\
\hline Unknown & $2(3.1)$ & $1(2.9)$ & $0.249^{c}$ \\
\hline \multicolumn{4}{|l|}{ Size of breast cancer pre-NAC (\%) } \\
\hline TO & $2(3.1)$ & 0 & \\
\hline $\mathrm{T} 1$ & $3(4.7)$ & $4(11.8)$ & \\
\hline $\mathrm{T} 2$ & $38(59.4)$ & $11(32.4)$ & \\
\hline T3 & $21(32.8)$ & $19(55.9)$ & $0.033^{c}$ \\
\hline \multicolumn{4}{|l|}{ Type of surgery (\%) } \\
\hline Breast-conserving surgery & $40(62.5)$ & $22(65.0)$ & \\
\hline Breast amputation & $23(35.9)$ & $16(35.0)$ & \\
\hline Other & $1(1.6)^{*}$ & 0 & $0.759^{c}$ \\
\hline \multicolumn{4}{|l|}{ Bloom-Richardson grade (\%) } \\
\hline Well differentiated & $9(14.1)$ & $3(8.8)$ & \\
\hline Moderately differentiated & $19(29.7)$ & $14(41.2)$ & \\
\hline Poorly differentiated & $8(12.5)$ & $6(17.6)$ & \\
\hline Unknown ${ }^{a}$ & $28(43.8)$ & $11(32.4)$ & $0.474^{c}$ \\
\hline Oestrogen receptor positive (\%) & $42(65.6)$ & $24(70.6)$ & $0.618^{c}$ \\
\hline Progesterone receptor positive (\%) & $33(51.6)$ & $19(55.9)$ & $0.724^{c}$ \\
\hline Her2Neu receptor positive (\%) & $27(42.2)$ & $4(11.8)$ & $0.006^{c}$ \\
\hline
\end{tabular}

RAD-: Patients without residual axillary disease at breast MRI following NAC; RAD+: Patients with residual axillary disease at breast MRI following NAC; ${ }^{a}$ Due to the use of NAC* BR-grade could not always be estimated; ${ }^{b}$ Mann-Whitney $\mathrm{U}$ test; ${ }^{\mathrm{C}} \mathrm{Chi}$-square test; ${ }^{*}$ Primary axillary breast cancer: ALND only.

\section{Resected lymph nodes}

No statistically significant difference was found between the two groups with respect to the mean number of lymph nodes removed during the axillary surgery, including ARM lymph nodes (mean 17.8 for RAD- group and 17.0 for RAD+ group, $P=0.532$, Table 9.2). The total number of positive lymph nodes, including ARM lymph nodes and the total number of positive ARM lymph nodes were significant higher in patients in the RAD+ group (Table 9.2). 
Table 2: Axillary status measured with MRI pre- and post-NAC and at pathological examination post-NAC, with and without residual axillary disease.

\begin{tabular}{|c|c|c|c|}
\hline Axillary lymph node status & $\begin{array}{l}\text { RAD- } \\
(n=64)\end{array}$ & $\begin{array}{l}\text { RAD+ } \\
(n=34)\end{array}$ & $P$ \\
\hline \multicolumn{4}{|l|}{ MRI data } \\
\hline Pre-NAC number of suspect nodes (Mean (Range)) & $1.9(0-8)$ & $2.6(1-8)$ & $0.018^{a}$ \\
\hline \multicolumn{4}{|l|}{ Post-NAC (\%) } \\
\hline No disease & $7^{c}(10.9)$ & - & \\
\hline Complete response & 57 (89.1) & - & \\
\hline Partial response & - & $29(85.3)$ & \\
\hline Stable disease & - & $4(11.8)$ & \\
\hline Progressive disease & - & $1(2.9)$ & \\
\hline \multicolumn{4}{|l|}{ Resected lymph nodes (Mean (Range)) } \\
\hline $\begin{array}{l}\text { Total number of lymph nodes removed, including ARM lymph } \\
\text { nodes }\end{array}$ & $17.8(4-44)$ & $17.0(9-28)$ & $0.532^{\mathrm{a}}$ \\
\hline Total number of ARM lymph nodes removed & $1.6(1-5)$ & $1.4(1-4)$ & $0.349^{a}$ \\
\hline $\begin{array}{l}\text { Total number of positive lymph nodes, including ARM lymph } \\
\text { nodes }\end{array}$ & $1.6(0-17)$ & $3.4(0-18)$ & $0.006^{\mathrm{a}}$ \\
\hline Total number of positive ARM lymph nodes & $0.1(0-2)$ & $0.3(0-2)$ & $0.007^{\mathrm{a}}$ \\
\hline \multicolumn{4}{|l|}{ Pathology (\%) } \\
\hline Metastasis in any lymph node & $25(39.1)$ & $24(70.6)$ & $0.003^{b}$ \\
\hline Axillary metastases without ARM metastases & $20(31.3)$ & $14(42.2)$ & $0.040^{b}$ \\
\hline Both axillary metastases and ARM metastases & $5(7.8)$ & $10(29.4)$ & $0.005^{\mathrm{b}}$ \\
\hline ARM metastases only without axillary metastases & 0 & 0 & \\
\hline
\end{tabular}

\section{Pathology}

Residual axillary disease following NAC was found at pathological analysis in 25 patients (39.1\%) in the RAD- group, and in 24 patients $(70.6 \%)$ in the RAD+ group $(P=0.003)$. The positive predictive value and negative predictive value of the post-NAC breast MRI for the detection of axillary lymph node metastases following NAC were $71 \%(95 \% \mathrm{Cl}: 52-84)$ and $60.9 \%(95 \% \mathrm{Cl}: 48-73)$, respectively.

Metastatic involvement of the ARM lymph nodes following NAC was present in five of the 64 patients $(7.8 \%)$ in the RAD- group and in 10 of the 34 patients $(29.4 \%)$ in the RAD + group ( $P=0.005$, Table 9.2 ). The positive predictive value and negative predictive value of the post-NAC breast MRI for the detection of positive ARM lymph nodes following NAC were $29 \%$ (95\%Cl: $16-48$ ) and $92 \%$ (95\%Cl: $82-97)$, respectively. None of the seven patients in whom no suspicious axillary lymph nodes were found on baseline MRI pre-NAC had metastatic involvement of the ARM lymph nodes. However, two of these patients had metastatic involvement of the other axillary lymph nodes. 


\section{Discussion}

To our knowledge, this is the first study reporting on the role of breast MRI in predicting the risk of metastatic involvement of ARM lymph nodes in the axilla. We found that breast MRI following NAC is not suitable to detect overall residual metastatic disease of the axilla, with a false negative rate of $39 \%$. However, this study demonstrates that a negative breast MRI with regard to presence of residual axillary disease following NAC, is accompanied by a rather low incidence of positive ARM lymph nodes (8\%).

In the RAD-group the incidence of pathological positive ARM lymph nodes in $\mathrm{cN}+$ patients following NAC was $7.8 \%$. This result compares favourably with the false negative ratio of sentinel node biopsy post-NAC $(6-21 \%)^{23-28}$ which currently is debated as a parameter to guide the need for any further axillary surgery. A low false negative rate as reported here may justify the possibility to preserve ARM lymph nodes in these patients without residual axillary disease on breast MRI in order to reduce morbidity. Proper follow up of these patients is however warranted in order to prevent them from exposure to higher risk of axillary recurrence. Especially in triple negative breast cancer patients, as they have little further systemic treatment options after failure of NAC in combination with surgery.

Since the introduction in 2007 the concept of the ARM procedure has been remained unchanged. ${ }^{9,10}$ In current literature high visualisation rates of ARM lymph nodes are reported in patients following NAC using blue dye, isotope or fluorescence.,14,29-31 Several studies have reported a low incidence of metastases in ARM lymph nodes in $\mathrm{cN}+$ patients treated with NAC. However, it remains a question of debate which of these patients are possible candidates to preserve these lymph nodes. ${ }^{12,14,31-36}$

As expected, the incidence of overexpression of Her2-neu was significantly higher in the RAD- group. This result explains the better overall response following Trastuzumab-based therapy in Her2-neu positive patients. ${ }^{22}$

A limitation of our study is that one might argue if a standard breast MRI is suitable for staging the axilla anyway. In literature positive predictive values of MRI are reported which range from $38 \%$ to $83 \% .{ }^{17,37}$ Our study showed a positive predictive value $71 \%$ of the post-NAC breast MRI for the detection of any axillary lymph node metastases following NAC, which demonstrates that breast MRI is rather unreliable to predict the axillary status following NAC. However, breast MRI may help to select a subgroup of $\mathrm{cN}+$ patients in whom ARM lymph nodes can be selectively spared during axillary lymph node dissection. Furthermore the use of a dedicated axillary coil and scanning protocol might further improve accuracy in the future. ${ }^{38}$ Recently Khandelwal et al. used US examination of the axilla pre- and post-NAC to determine the axillary response. In this study of 51 patients none of the ARM lymph nodes contained metastatic involvement in $\mathrm{cN}+$ patients with a complete or partial axillary 
response to NAC. ${ }^{39}$ These results indicate that the specificity and positive predictive value of axillary lymph node staging may be improved with the additional use of US. ${ }^{40}$ In conclusion, breast MRI following NAC is not suitable to detect residual metastatic disease of the axilla. However, breast MRI post-NAC may be of use to identify cN+ patients with a low risk of ARM lymph node metastases. This may help to select a subgroup of $\mathrm{cN}+$ patients in whom sparing of ARM lymph nodes during axillary lymph node dissection can be considered, in a way to reduce the risk of postoperative morbidity. Further research, preferably a randomized control trial, in these selected patients (post-NAC cN+ patients) is needed to provide evidence whether preservation of ARM lymph nodes during ALND is safe with respect to regional disease control and as an effort to reduce morbidity. 


\section{References}

1. van de Vijver MJ, He YD, van't Veer LJ, Dai H, Hart AA, Voskuil DW, et al. A gene-expression signature as a predictor of survival in breast cancer. N Engl J Med. 2002;347:1999-2009.

2. Goldhirsch A, Glick JH, Gelber RD, Senn HJ. Meeting highlights: International Consensus Panel on the Treatment of Primary Breast Cancer. J Natil Cancer Inst. 1998;90:1601-8.

3. Tausch C, Baege A, Dietrich D, Vergin I, Heuer H, Heusler RH, et al. Can axillary reverse mapping avoid lymphedema in node positive breast cancer patients? Eur J Surg Oncol. 2013;39:880-6.

4. Noguchi M, Morioka E, Ohno Y, Noguchi M, Nakano Y, Kosaka T. The changing role of axillary lymph node dissection for breast cancer. Breast Cancer. 2013;20:41-6.

5. Liu CQ, Guo Y, Shi JY, Sheng Y. Late morbidity associated with a tumour-negative sentinel lymph node biopsy in primary breast cancer patients: a systematic review. Eur J Cancer. 2009;457:1560-8.

6. Noguchi M, Miwa K, Michigishi T, Yokoyama K, Nishijima H, Takanaka T, et al. The Role of Axillary Lymph Node Dissection in Breast Cancer Management. Breast Cancer. 1997;4:143-53.

7. Klimberg VS. A new concept toward the prevention of lymphedema: axillary reverse mapping. J Surg Oncol. 2008;97:563-4.

8. Nos C, Kaufmann G, Clough KB, Collignon MA, Zerbib E, Cusumano P, et al. Combined axillary reverse mapping (ARM) technique for breast cancer patients requiring axillary dissection. Ann Surg Oncol. 2008;15:2550-5.

9. Nos C, Lesieur B, Clough KB, Lecuru F. Blue dye injection in the arm in order to conserve the lymphatic drainage of the arm in breast cancer patients requiring an axillary dissection. Ann Surg Oncol. 2007; 14:2490-6.

10. Thompson M, Korourian S, Henry-Tillman R, Adkins L, Mumford S, Westbrook KC, et al. Axillary reverse mapping (ARM): a new concept to identify and enhance lymphatic preservation. Ann Surg Oncol. 2007;14:1890-5.

11. Klompenhouwer EG, Gobardhan PD, Beek MA, Voogd AC, Luiten EJ. The clinical relevance of axillary reverse mapping (ARM): study protocol for a randomized controlled trial. Trials. 2013;14:111.

12. Gobardhan PD, Wijsman JH, van Dalen T, Klompenhouwer EG, van der Schelling GP, Los J, et al. ARM: axillary reverse mapping - the need for selection of patients. Eur J Surg Oncol. 2012;38:657-61.

13. Beek MA, Gobardhan PD, Klompenhouwer EG, Rutten HJ, Voogd AC, Luiten EJ. Axillary reverse mapping (ARM) in clinically node positive breast cancer patients. Eur J Surg Oncol. 2015;41:59-63.

14. Noguchi M, Noguchi M, Nakano $\mathrm{Y}$, Ohno $\mathrm{Y}$, Kosaka T. Axillary reverse mapping using a fluorescence imaging system in breast cancer. J Surg Oncol. 2012;105:229-34.

15. Deng H, Chen L, Jia W, Chen K, Zeng Y, Rao N, et al. Safety study of axillary reverse mapping in the surgical treatment for breast cancer patients. J Cancer Res Clin Oncol. 2011;137:1869-74.

16. Scaranelo AM, Eiada R, Jacks LM, Kulkarni SR, Crystal P. Accuracy of unenhanced MR imaging in the detection of axillary lymph node metastasis: study of reproducibility and reliability. Radiology. 2012;262:425-34.

17. Javid S, Segara D, Lotfi P, Raza S, Golshan M. Can breast MRI predict axillary lymph node metastasis in women undergoing neoadjuvant chemotherapy. Ann Surg Oncol. 2010;17:1841-6.

18. Mortellaro VE, Marshall J, Singer L, Hochwald SN, Chang M, Copeland EM, et al. Magnetic resonance imaging for axillary staging in patients with breast cancer. J Magn Reson Imaging. 2009;30:309-12.

19. Kvistad KA, Rydland J, Smethurst HB, Lundgren S, Fjosne HE, Haraldseth O. Axillary lymph node metastases in breast cancer: preoperative detection with dynamic contrast-enhanced MRI. Eur Radiol. 2000;10:1464-71.

20. Elston CW, Ellis IO. Pathological prognostic factors in breast cancer. I. The value of histological grade in breast cancer: experience from a large study with long-term follow-up. Histopathology. 1991;19:403-10.

21. NOABON. Landelijke richtlijn mammacarcinoom 2.0. wwwoncolinenl/mammacarcinoom.

22. Fuchs EM, Kostler WJ, Horvat R, Hudelist G, Kubista E, Attems J, et al. High-level ERBB2 gene amplification is associated with a particularly short time-to-metastasis, but results in a high rate of 
complete response once trastuzumab-based therapy is offered in the metastatic setting. Int J Cancer. 2014;135:224-31.

23. Alvarado R, Yi M, Le-Petross $H$, Gilcrease $M$, Mittendorf EA, Bedrosian I, et al. The role for sentinel lymph node dissection after neoadjuvant chemotherapy in patients who present with node-positive breast cancer. Ann Surg Oncol. 2012;19:3177-84.

24. Canavese G, Dozin B, Vecchio C, Tomei D, Villa G, Carli F, et al. Accuracy of sentinel lymph node biopsy after neo-adjuvant chemotherapy in patients with locally advanced breast cancer and clinically positive axillary nodes. Eur J Surg Oncol. 2011;37:688-94.

25. Hunt KK, Yi M, Mittendorf EA, Guerrero C, Babiera GV, Bedrosian I, et al. Sentinel lymph node surgery after neoadjuvant chemotherapy is accurate and reduces the need for axillary dissection in breast cancer patients. Ann Surg. 2009;250:558-66.

26. Kelly AM, Dwamena B, Cronin P, Carlos RC. Breast cancer sentinel node identification and classification after neoadjuvant chemotherapy-systematic review and meta analysis. Acad Radiol. 2009;16:551-63.

27. van Deurzen $C H$, Vriens $B E$, Tjan-Heijnen $V C$, van der Wall $E$, Albregts $M$, van Hilligersberg $R$, et al. Accuracy of sentinel node biopsy after neoadjuvant chemotherapy in breast cancer patients: a systematic review. Eur J Cancer. 2009;45:3124-30.

28. Xing Y, Foy M, Cox DD, Kuerer HM, Hunt KK, Cormier JN. Meta-analysis of sentinel lymph node biopsy after preoperative chemotherapy in patients with breast cancer. Br J Surg. 2006;93:539-46.

29. Rubio IT, Cebrecos I, Peg V, Esgueva A, Mendoza C, Cortadellas T, et al. Extensive nodal involvement increases the positivity of blue nodes in the axillary reverse mapping procedure in patients with breast cancer. J Surg Oncol. 2012;106:89-93.

30. Boneti C, Badgwell B, Robertson Y, Korourian S, Adkins L, Klimberg V. Axillary reverse mapping (ARM): initial results of phase $\mathrm{II}$ trial in preventing lymphedema after lymphadenectomy. Minerva Ginecologica. 2012;64:421-30.

31. Ikeda K, Ogawa Y, Kajino C, Deguchi S, Kurihara S, Tashima T, et al. The influence of axillary reverse mapping related factors on lymphedema in breast cancer patients. Eur J S Oncol. 2014;40:818-23.

32. Schunemann E, Jr., Doria MT, Silvestre JB, Gasperin P, Jr., Cavalcanti TC, Budel VM. Prospective study evaluating oncological safety of axillary reverse mapping. Ann Surg Oncol. 2014;21:2197-202.

33. Ikeda K, Ogawa Y, Komatsu H, Mori Y, Ishikawa A, Nakajima T, et al. Evaluation of the metastatic status of lymph nodes identified using axillary reverse mapping in breast cancer patients. World J Surg Oncol. 2012;10:233.

34. Noguchi M, Yokoi M, Nakano Y. Axillary reverse mapping with indocyanine fluorescence imaging in patients with breast cancer. J Surg Oncol. 2010;101:217-21.

35. Yue T, Zhuang D, Zhou P, Zheng L, Fan Z, Zhu J, et al. A Prospective study to assess the feasibility of axillary reverse mapping and evaluate its effect on preventing lymphedema in breast cancer patients. Clin Breast Cancer. 2015;15:301-6.

36. Bedrosian I, Babiera GV, Mittendorf EA, Kuerer HM, Pantoja L, Hunt KK, et al. A phase I study to assess the feasibility and oncologic safety of axillary reverse mapping in breast cancer patients. Cancer. 2010;116:2543-8.

37. Rahbar H, Conlin JL, Parsian S, DeMartini WB, Peacock S, Lehman CD, et al. Suspicious axillary lymph nodes identified on clinical breast $\mathrm{MRI}$ in patients newly diagnosed with breast cancer: can quantitative features improve discrimination of malignant from benign? Acad Radiol. 2015;22:430-8.

38. Kuijs VJ, Moossdorff M, Schipper RJ, Beets-Tan RG, Heuts EM, Keymeulen KB, et al. The role of MRI in axillary lymph node imaging in breast cancer patients: a systematic review. Insights Imaging. 2015;6:203-15.

39. Khandelwal R, Poovamma CU, Shilpy C, Prema M, Anthony P. Axillary reverse mapping: Is it feasible in locally advanced breast cancer patients? Breast Dis. 2014;34:151-5.

40. Abe H, Schacht D, Kulkarni K, Shimauchi A, Yamaguchi K, Sennett CA, et al. Accuracy of axillary lymph node staging in breast cancer patients: an observer-performance study comparison of MRI and ultrasound. Acad Radiol. 2013;20:1399-404. 





\section{Summary and general discussion}

Axillary lymph node dissection (ALND) has been standard of care in axillary treatment of lymph node positive breast cancer patients for many decades. One of the most important reasons to critically re-evaluate the need for ALND was the postoperative morbidity associated with this procedure, especially upper extremity lymphedema. ${ }^{1-8}$ The basic question as to which mechanisms underlie the development of upper extremity lymphedema, as well as the development of surgical refinements of ALND in an effort to prevent this did not gain much attention until publications of Nos et al., Thompson et al. and Klimberg in 2007, who introduced axillary reverse mapping (ARM). ${ }^{9-11}$ This thesis includes a variety of clinical studies on ARM performed in our hospital.

Following a long-lasting period in which extensive axillary surgery was considered the gold standard for both diagnostic and therapeutic purposes, a major shift towards less invasive axillary lymph node surgery occurred over a period of approximately two decades. Chapter 2 contains the results of a large population-based study. In this study we describe the patterns of care in the treatment of the axilla and evaluate the impact on daily clinical practice of several important scientific landmarks, including the introduction of the sentinel lymph node biopsy (SLNB) in 1998 and the publication of both the American College of Surgeons Oncology Group (ACOSOG) Z0011 trial and the European Organisation for Research and Treatment of Cancer (EORTC) AMAROS trial. $^{12,13}$

The SLNB was included in the Dutch national breast cancer treatment guideline in 1999. ${ }^{14,15}$ Currently SLNB (the gold standard for clinically lymph node negative breast cancer patients) is carried out in more than $70 \%$ of all patients in the Netherlands. Consequently, the proportion of women undergoing primary ALND has decreased from $89 \%$ in $1993-1994$ to $12 \%$ in $2013-2014$.

The publication of the ACOSOG Z0011 trial in 2011 can be considered as a practicechanging event in the management of the axilla. ${ }^{12}$ This trial randomised breast cancer patient with one to three positive sentinel lymph nodes (SLN) to axillary surgery or a wait and see policy. After a median follow up of 6.3 years there was no significant difference in overall and disease free survival between the two groups. Prior to the publication of the ACOSOG Z0011 trial only 8\% of the patients with one to three positive lymph nodes treated with breast-conservation surgery (one of the main inclusion criteria of the ACOSOG Z0011 trial) were staged by SLNB only compared to $50 \%$ in the period 2013-2014. However, the ACOSOG Z0011 trial has been criticized for several reasons. First, the expected accrual of this trial was 1900 patients, but the actual recruitment was more than $50 \%$ lower (891 patients). Second, patients were recruited form 115 different participating centres and some $20 \%$ of the patients were lost to follow-up. ${ }^{16}$ Third, the included patients had a rather low tumour burden; only 
$27 \%$ had additional metastases in lymph nodes removed by completion ALND, as compared to $40 \%$ in other large patient series. ${ }^{17}$ Fourth, the rate of patients with micro-metastatic lymph node involvement was remarkably high and differed significantly between both treatment arms (44.8\% in the SLNB only group and $37.5 \%$ in the ALND group, $p<0.05)$. Fifth, patients treated with neoadjuvant chemotherapy (NAC) were excluded. Sixth, approximately $97 \%$ of all patients received some kind of adjuvant systemic treatment (chemotherapy and/or hormonal therapy) which is known to significantly reduce the risk of local and regional recurrence. ${ }^{18}$ Finally, all patients received tangential field irradiation of the breast in the framework of breastconserving therapy, which covers at least the lower part of the axilla. ${ }^{16,19,20}$

Recently, the AMAROS trial and the International Breast Cancer Study Group (IBCSG) 23-01 trial $^{21}$ have also challenged the need for completion ALND in patients with SLN metastases. The AMAROS trial showed that omitting completion ALND does not result in inferior overall- and disease free survival in CT1-2NO breast cancer patients with one to two SLN metastases, compared to axillary radiotherapy. The IBCSG 23-01trial showed that completion ALND can be safely omitted in SLN positive breast cancer patients with one or more micro-metastatic positive SLN's. Prior to the publication of the AMAROS trial in 2014, and possibly due to the early reports of the results at the annual meeting of the American Society of Clinical Oncology (ASCO) in 2013, we observed that completion ALND was also omitted in an increasing number of patients with one to three positive SLN's undergoing mastectomy. In 2013-2014 22\% of these patients were staged by SLNB only (with addition of axillary radiotherapy), in comparison with the $6 \%$ prior to the publication. However, the AMAROS trial has also been criticized for several reasons. First, there were fewer axillary recurrences than predicted and therefore the trial was not sufficiently powered to address the primary endpoint, which was non-inferiority with respect to the risk of axillary recurrence at five years. Second, $40 \%$ of the patients could have been treated without completion ALND or axillary radiotherapy according to the results of the IBCSG 23-01 trial because of their N1mic (micrometastatic disease) or Nitc (isolated tumour cells) status. ${ }^{21}$ Third, patients treated with NAC were excluded. Fourth, approximately $77 \%$ of all patients received some kind of adjuvant systemic treatment (chemotherapy and/or hormonal therapy). Finally, although the incidence of upper extremity lymphedema, identified by circumference measurement, was reported to be lower in the radiotherapy group. The quality of life however did not differ between the two groups. ${ }^{22}$

As stated above, around 2007 a new technique was introduced to map the upper extremity lymph nodes and lymphatics in the axilla: axillary reverse mapping (ARM). ${ }^{9-11}$ The fundamental concept of the ARM procedure is based on the assumption that the upper extremity and the breast each have their own discrete pathways of lymphatic drainage in the axilla. In chapter 3 we explained this new technique and reported the first results of a feasibility study. We showed that ARM 
using blue dye is feasible with a visualization rate ranging from $86 \%$ to $94 \%$. Of these patients, $46 \%$ had an indication for ALND based on a positive SLNB (SLN+ group) and the remaining $54 \%$ underwent primary ALND because of fine needle aspiration cytology (FNAC) proven axillary metastases (CPN+ group). In the first group all ARM lymph nodes were tumour-free, as compared to $22 \%$ in the latter group. ${ }^{23}$

Since the introduction of ARM using blue dye, various reports using different visualisation techniques have been published. Chapter $\mathbf{4}$ provides an update regarding ARM in breast cancer patients. In current literature three visualisation techniques have been described; blue dye, radio-isotopes (Tc-99) and fluorescent imaging. If upper extremity lymphedema is caused by disruption of ARM lymphatics and/or removal of ARM lymph nodes, mapping the axilla using Tc-99 alone seems rather impractical because of invisibility of lymphatics. Visualisation using blue dye is preferable since it is easy to perform and inexpensive. However, by using blue dye, a "blue tattoo" will be left on the patients skin following subcutaneous injection, which disappears after a period of several weeks to more than a year. Visualisation using fluorescent imaging seems to result in high visualisation rates of both ARM lymph nodes and lymphatics. However, this technique requires the use of more expensive equipment and the experience is limited resulting in only three study groups have reported using this technique. ${ }^{24-26}$ Furthermore, in SLN+ patients it is oncologically safe to preserve the ARM lymph nodes precluded that no convergence of the ARM lymph nodes and SLN is present. In CPN+ patients some studies have reported metastatic involvement of the ARM lymph nodes, casting doubt over the oncological safety of preserving ARM lymph nodes in this subgroup. A further refinement of selection of the CPN+ patients who may be candidate for sparing upper extremity lymph nodes and corresponding lymphatics by means of ARM is warranted in order not to compromise oncological safety.

In chapter $\mathbf{5}$ we present the design for a multicentre randomized controlled trial to determine the clinical relevance and safety of selectively sparing upper-limb axillary lymph nodes and their corresponding lymphatics by means of ARM. The aim of the ARM trial is to determine the clinical utility of the ARM technique in identifying and sparing the upper limb-related axillary lymph nodes and its subsequent reduction of lymphedema of the upper limb. After determining the indication for a completion ALND, merely based on a positive SLN, patients will be randomly allocated to one of two groups: Level I-II ALND with preservation of the upper limb-related axillary lymph nodes (ARM-ALND) and a standard Level I-II ALND (standard-ALND). Patients with an indication for primary ALND, based on axillary metastases proven by pre-operative fine needle aspiration cytology (FNAC, CPN+ patients), can be included in the registration study to confirm the feasibility of the procedure and to allow further analysis. 
The primary outcome of the ARM trial will be the occurrence of lymphedema in the upper limb. Lymphedema will be measured at 6, 12 and 24 months postoperatively, using three techniques: the water-displacement method, circumference measurement method and questionnaires. Chapter 6 examines the reliability and the reproducibility of the "Inverse Water Volumetry apparatus" (IWV-apparatus) for the measurement of upper extremity volumes. This study demonstrated that the use of the IWV-apparatus for the measurement of repeated upper extremity volumes can be considered a reliable and reproducible method. There was no inter-observer variability and hardly any intra-observer and intra-subject variability. The intra class correlation coefficient defined as the ratio of the inter-subject component to the total variance reached the almost perfect value of 0.99 which is similar to the results reported in a study by Damstra et al.. ${ }^{27}$

There is still an ongoing debate on the necessity of performing completion ALND in SLN+ patients. The ACOSOG Z0011 trial indicated that completion ALND could be avoided in CT1-2NO breast cancer patients with one to three SLN metastases treated with breast-conserving therapy, including irradiation of the breast, as well as adjuvant systemic therapy. Furthermore the AMAROS trial indicated that completion ALND could be replaced by axillary radiotherapy in pT1-2 breast cancer patients with macrometastases found in the SLN. However, in selected breast cancer patients with a positive SLN a completion ALND may still be the treatment of choice, for instance for those in whom mastectomy is accompanied by direct reconstruction with a tissue expander or patients with high loads of residual axillary disease following NAC. ${ }^{28}$

Chapter 7 includes a joined vision concerning the clinical utility of ARM in an era of changing perceptions concerning axillary surgery. The AMAROS trial has compared axillary radiotherapy with a standard-ALND. ${ }^{13}$ The results of this study suggest that axillary radiotherapy is a safe treatment option causing less upper extremity lymphedema compared to standard-ALND. However axillary radiotherapy in breast cancer patients is not harmless and also carries its specific risks, such as shoulder problems and toxic effects to other organs. Selectively sparing upper-limb axillary lymph nodes and lymphatic drainage by means of ARM during ALND may have the potential to reduce the incidence of upper extremity lymphedema to much lower levels than the figures that are reported using the classical technique.

Primary ALND is still the treatment of choice in CPN+ patients. ${ }^{29-31}$ Several studies reported on the incidence of metastatic involvement of the ARM lymph nodes ranging from $0 \%$ to $60 \%$ in $\mathrm{CPN}+$ patients. ${ }^{9,32}$ Further research is needed to select which of these patients are possible candidates to preserve these upper-limb axillary lymph nodes and their corresponding lymphatics by means of ARM. 
In chapter 8 we prospectively evaluated the incidence of metastatic involvement of ARM lymph nodes in CPN+ patients who underwent ALND following NAC (NAC+ group) compared to patients who underwent ALND without NAC (NAC- group). The study population included a consecutive series of 112 female breast cancer patients who received a primary ALND between October 2009 and November 2013. Metastatic involvement of the ARM-nodes was found in13 of 79 patients $(17 \%)$ in the NAC+ group compared to 7 of 19 patients (37\%) in the NAC- group $(P=0.048)$. This result suggests that some $\mathrm{CPN}+$ patients may be candidates for the ARM-procedure, provided that they are receiving NAC.

In chapter 9 we retrospectively evaluated metastatic involvement of ARM lymph nodes in CPN+ patients who received NAC followed by primary ALND. Patients without residual axillary disease at breast MRI following NAC (64 patients) were compared with patients with residual axillary disease (34 patients). Presence of suspect axillary lymph nodes on pre- and post-NAC breast MRI was determined by two experienced breast radiologists and correlated to histopathological findings. This study showed that the proportion of patients with metastatic involvement of the ARM lymph nodes following NAC significantly differed between both groups; 8\% (5/64) in the patients without radiological signs of residual axillary disease versus $29 \%(10 / 34)$ in the patients with residual disease $(P=0.005)$. Concluding that breast MRI post-NAC may be of use to identify $\mathrm{CPN}+$ patients with a low risk of ARM lymph node metastases.

\section{Future perspectives: Towards personalised axillary treatment}

The therapeutic effect of the ALND was believed to increase overall survival and to decrease axillary recurrence. In a meta-analysis published in 1999 of 3000 clinically lymph node negative breast cancer patients Orr showed that prophylactic ALND improved overall survival, ranging from $4 \%$ to $16 \%{ }^{33}$. A limitation of this meta-analyse is that in the included time period none of the patients had been treated with adjuvant systemic therapy. This is in sharp contrast with the more frequent use of chemotherapy in current clinical practice. On the other hand, after 10 years of followup the European Organization for Research and Treatment of Cancer (EORTC) $22922 / 10925$ trial also reported a (marginal significant) effect on overall survival in breast cancer patients treated by ALND and regional nodal irradiation. ${ }^{34}$ Furthermore the National Surgical Adjuvant Breast and Bowel Project (NSABP) B-04 trial and the NCIC Clinical Trials Group (CTG) MA.20 trial both reported a significantly lower regional recurrence rate after axillary treatment. ${ }^{17,35}$ The NSABP B-04 trial reported after 25 years of follow-up a significantly lower regional recurrence rate, in clinically node negative breast cancer patients treated by radical mastectomy (mastectomy 
with ALND) or mastectomy with postoperative radiation versus mastectomy only. The NCIC-CTG MA.20 trial showed in CPN+ patients or high-risk lymph node negative breast cancer patients that the addition of regional nodal irradiation to whole-breast irradiation reduced the rate of breast cancer recurrence. However, the use of systemic therapy (chemotherapy and/or hormonal therapy), which is currently administered more frequently and with increasing effectiveness has ameliorated survival rates in patients with breast cancer and is also known to substantially decrease local and regional recurrence rates. ${ }^{18}$

\section{Clinically node negative disease}

Based on the low incidence of occult (macro)metastases in cT1-2NO breast cancer patients treated with breast-conserving therapy the current Dutch BOOG 2013-08 trial randomises clinically node negative breast cancer patients undergoing breastconserving therapy for lumpectomy with SLNB versus lumpectomy only. The results of this study will help to define if the SLNB can be safely omitted in clinically node negative breast cancer patients undergoing breast-conserving (Figure 10.1).

\section{Sentinel lymph node positive disease}

Once a positive SLN is found clinicians are nowadays faced with several therapeutic options.

The International Breast Cancer Study Group (IBCSG) 23-01 trial has shown that completion ALND can be safely omitted in clinically node negative patients with one or more micrometastatic positive $\operatorname{SLN}(\mathrm{s})$ irrespective of whether breast-conserving therapy or mastectomy has been performed. ${ }^{21}$

Recently the results of the ACOSOG Z0011 trial showed that omitting completion ALND did not result in inferior overall- and disease free survival in cT1-2NO breast cancer patients with one to three SLN micro- or even macro-metastases treated by breast-conserving therapy, which includes standard irradiation of the breast. Since most of these patients did also receive adjuvant systemic therapy, based on their tumour characteristics and SLN positive disease, systemic treatment is currently regarded as a prerequisite when omitting completion ALND is considered in this subgroup of patients.

The results of the AMAROS trial showed that for patients with pT1-2 primary breast cancer, without palpable lymphadenopathy, axillary irradiation is not inferior to standard-ALND in SLN+ patients with regard to overall- and disease free survival. ${ }^{13}$ Despite some possible limitations as addressed earlier in this discussion this international multicentre trial has delivered a major contribution to our current way of thinking about the management of the axilla in SLN+ patients and indicates that axillary radiotherapy is the preferred treatment option because of the lower risk of 
upper extremity lymphedema. However, the forthcoming results of the ARM study may shed a new light on the role of additional axillary surgery in SLN+ patients.

Finally, there is an ongoing discussion if "any" axillary treatment in SLN+ patients treated with adjuvant systemic treatment is needed in case of administration of systemic therapy which is known to have major impact also on disease free survival. ${ }^{18,36,37}$ Therefore, the UK POSNOC (POsitive Sentinel NOde: adjuvant therapy alone versus adjuvant therapy plus Clearance or axillary radiotherapy) trial and the BOOG 2013-07 trial (The value of completion axillary treatment (ALND or axillary radiotherapy) in SLN+ patients undergoing a mastectomy) will help to further define the role of any axillary treatment in patients with macrometastases found in the SLN. The UK POSNOC trial includes patients with unifocal or multifocal invasive breast cancer, largest primary lesion $\leq 5 \mathrm{~cm}$ and one or two macrometastases in the SLN(s) and the BOOG 2013-07 trial includes patients with unilateral invasive cT1-2NO breast cancer treated with mastectomy, with limited SLN metastases (one micro- to three macrometastatic SLN(s)).

On the other hand, if the indication for systemic therapy is made before surgery, the administration of systemic therapy is increasingly shifting towards the neoadjuvant setting. This shift in treatment sequence has faced clinicians with the question as to when to perform a SLNB, i.e. before or after NAC in clinically node negative patients. ${ }^{38}$ Up to now, a completion ALND is still the gold standard in breast cancer patients with macrometastases found in the SLN prior to NAC, irrespective of the axillary response. On the other hand, in one out of three patients complete pathologic axillary response is achieved for breast cancer patients treated with NAC. ${ }^{39,40}$ Therefore, in retrospect, standard performance of an ALND could be considered overtreatment in these patients. However, in patients in whom only partial or even no axillary response is observed following NAC, further axillary treatment is warranted. Therefore, a logical step seems to perform a SLNB following NAC in order to properly select patients with possible remnant axillary disease. However the clinical accuracy of a post-NAC SLNB is subject of discussion since a wide range of false negative rates have been reported $(6-21 \%)^{39,41-45}$

Since the combination of axillary radiotherapy and axillary surgery (i.e. ALND) is notorious for its high risk of upper extremity lymphedema (46), axillary radiotherapy may be preferred over completion ALND in case of an absolute indication for postoperative radiotherapy. However there will always be patients in whom axillary surgery is preferred over axillary radiotherapy. For instance when mastectomy is accompanied by direct reconstruction with a tissue expander or patients with high loads of residual axillary disease following NAC. ${ }^{28}$

In conclusion, there are several therapeutic options for the treatment of the axilla in SLN+ patients; a completion ALND, a ALND-ARM (currently ARM trial), axillary radiotherapy (AMAROS trial) or a wait and see policy (ACOSOG Z0011 trial and BOOG07 trial; Figure 10.1). 
Further studies are needed to provide sufficient evidence for staging SLN+ patients with respect to concurrent neoadjuvant systemic therapy and ideally the ACOSOG Z0011 trial and AMAROS trial are repeated in post-NAC SLN+ patients to provide level 1 evidence for extending the therapeutic options; wait and see (ACOSOG Z0011) of axillary radiotherapy (AMAROS) in these selected group of patients.

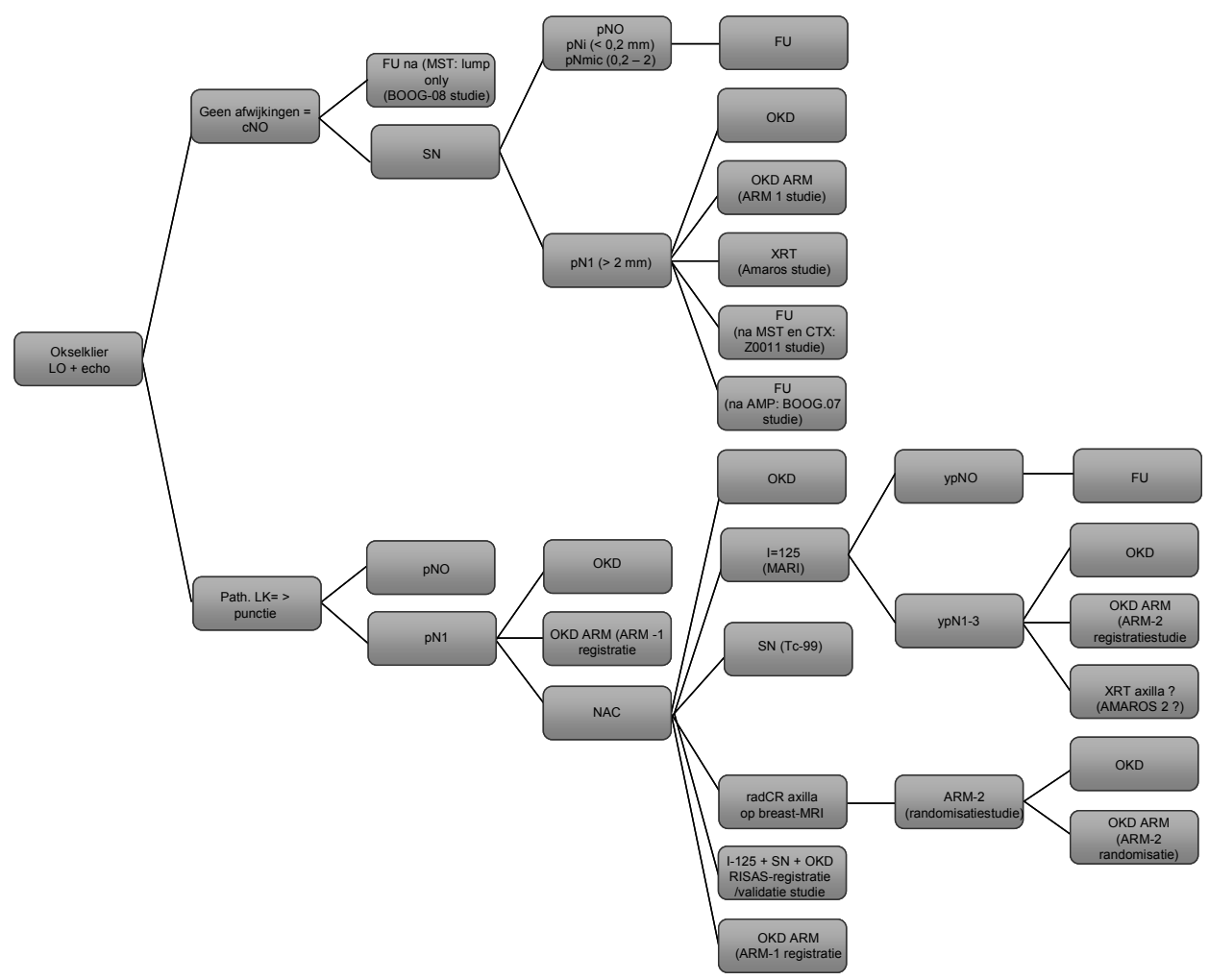

Figure 10.1 Axillary treatment 2016

\section{Clinically node positive disease}

Currently, ALND is still the gold standard in CPN+ patients and most of these patients will also be treated with adjuvant systemic therapy. ${ }^{29-31}$ As mentioned earlier, once the indication for systemic therapy is set, the timing of administration is increasingly shifted to neoadjuvant instead of adjuvant. Bearing in mind that up to $40 \%$ pathologic complete axillary response is achieved in lymph node positive disease, performing a SLNB post-NAC seems a logical step in order to properly select patients with possible 
residual axillary disease. As mentioned earlier, the clinical accuracy of a post-NAC SLNB is subject of discussion since a wide range of false negative rates is reported (6$21 \%) .^{39,41-45}$ Recently, Donker et al. proposed using marking of a single axillary positive lymph node, in CPN+ patients, to guide as representative predictor of the remaining axillary node status following NAC. This procedure is also known as the MARI procedure (Marking Axillary lymph nodes with Radioactive lodine seeds) and they reported a false negative ratio of $7 \% .{ }^{47}$ Nevertheless, complete pathologic axillary response in the MARI lymph node was seen in 30 patients and 5 of these 30 patients (17\%) had residual tumour burden in the ALND. This indicates that in one out of every six patients, with a pathologic axillary response in the SLNB, we would still leave possible residual axillary involved lymph nodes behind. Recently, the ACOSOG Z1071 trial demonstrated that a decrease in false negative ratio of post-NAC SLNB can be achieved when three or four SLNs are removed (instead of one or two). ${ }^{48}$ The latter may give rise to a random level I node picking when only 1 blue hot node is found by dual tracer technique.

When discussing the clinical impact of a false negative result of SLNB it is worth mentioning that residual axillary disease following NAC may have a different clinical impact as compared to a false-negative test result in untreated disease. In order to more accurately predict the residual axillary tumour burden after NAC a new concept will be subject of a new study, the RISAS procedure (Radioactive lodine Seed localisation in the Axilla in CPN+ patients combined with SLNB following NAC). In this trial, CPN+ patients treated with NAC will undergo the RISAS procedure and in order to validate this concept, a simultaneous ALND will be carried out. If RISAS is proven to be accurate, completion ALND can be omitted if axillary pathologic axillary response is detected. The RISAS study will start in the beginning of 2016.

Currently, there is a trend towards replacement of ALND in CPN+ patients following NAC by axillary radiotherapy or even abandoning ALND. Clinical evidence for these options is lacking however and ideally the AMAROS trial would have to be repeated in CPN+ patients treated with NAC; the "AMAROS-2" trial.

Recently, the results of the ARM registration study showed that breast MRI post-NAC may help to select a subgroup of CPN+ patients in whom sparing of ARM lymph nodes during ALND can be considered, in a way to reduce the risk of postoperative morbidity (chapter 9). Further research, preferably again a randomized control trial, in CPN+ patients is needed to provide evidence whether preservation of ARM lymph nodes during ALND is safe with respect to regional disease control and as an effort to reduce morbidity.

In conclusion, ALND has lost its long standing monopoly in the treatment of SLN+ patients and CPN+ breast cancer patients. Axillary treatment options have increased from a "one size fits all" to a more personalised axillary treatment as demonstrated in Figure 10.1 . 
Several new studies will have to be designed in order to further elucidate their respective role in an effort to further de-escalate additional axillary interventions thereby reducing unnecessary morbidity however with preservation of our current achievements in regional disease control. 


\section{References}

1. Schijven MP, Vingerhoets AJ, Rutten HJ, Nieuwenhuijzen GA, Roumen RM, van Bussel ME, et al. Comparison of morbidity between axillary lymph node dissection and sentinel node biopsy. Eur J Surg Oncol. 2003;29:341-50.

2. Blanchard DK, Donohue JH, Reynolds C, Grant CS. Relapse and morbidity in patients undergoing sentinel lymph node biopsy alone or with axillary dissection for breast cancer. Arch Surg. 2003;138:482-7.

3. Haid A, Koberle-Wuhrer R, Knauer M, Burtscher J, Fritzsche H, Peschina W, et al. Morbidity of breast cancer patients following complete axillary dissection or sentinel node biopsy only: a comparative evaluation. Breast Cancer Res Treat. 2002;73:31-6.

4. Leidenius M, Leivonen M, Vironen J, von Smitten K. The consequences of long-time arm morbidity in node-negative breast cancer patients with sentinel node biopsy or axillary clearance. J Surg Oncol. 2005;92:23-31.

5. Mansel RE, Fallowfield L, Kissin M, Goyal A, Newcombe RG, Dixon JM, et al. Randomized multicenter trial of sentinel node biopsy versus standard axillary treatment in operable breast cancer: the ALMANAC Trial. J Natl Cancer Inst. 2006;98:599-609.

6. Ronka R, von Smitten K, Tasmuth T, Leidenius M. One-year morbidity after sentinel node biopsy and breast surgery. Breast. 2005;14:28-36.

7. Schrenk $P$, Rieger R, Shamiyeh A, Wayand W. Morbidity following sentinel lymph node biopsy versus axillary lymph node dissection for patients with breast carcinoma. Cancer. 2000 Feb 1;88(3):608-14.

8. Swenson KK, Nissen MJ, Ceronsky C, Swenson L, Lee MW, Tuttle TM. Comparison of side effects between sentinel lymph node and axillary lymph node dissection for breast cancer. Ann Surg Oncol. 2002;9:745-53.

9. Nos C, Lesieur B, Clough KB, Lecuru F. Blue dye injection in the arm in order to conserve the lymphatic drainage of the arm in breast cancer patients requiring an axillary dissection. Ann Surg Oncol. 2007;14:2490-6.

10. Thompson M, Korourian S, Henry-Tillman R, Adkins L, Mumford S, Westbrook KC, et al. Axillary reverse mapping (ARM): a new concept to identify and enhance lymphatic preservation. Ann Surg Oncol. 2007;14:1890-5.

11. Klimberg VS. A new concept toward the prevention of lymphedema: axillary reverse mapping. J Surg Oncol. 2008;97:563-4.

12. Giuliano AE, Hunt KK, Ballman KV, Beitsch PD, Whitworth PW, Blumencranz PW, et al. Axillary dissection vs no axillary dissection in women with invasive breast cancer and sentinel node metastasis: a randomized clinical trial. JAMA. 2011;305:569-75.

13. Donker M, van Tienhoven G, Straver ME, Meijnen P, van de Velde CJ, Mansel RE, et al. Radiotherapy or surgery of the axilla after a positive sentinel node in breast cancer (EORTC 10981-22023 AMAROS): a randomised, multicentre, open-label, phase 3 non-inferiority trial. Lancet Oncol. 2014;15:1303-10.

14. Roumen RM, Pijpers HJ, Thunnissen FB, Ruers TJ. [Summary of the guideline 'Sentinel node biopsy in breast cancer.' Dutch Work Group 'Sentinel Node Biopsy for Breast Cancer']. Ned Tijdschr Geneeskd. 2000;144:1864-7.

15. Ho VK, van der Heiden-van der Loo M, Rutgers EJ, van Diest PJ, Hobbelink MG, Tjan-Heijnen VC, et al. Implementation of sentinel node biopsy in breast cancer patients in the Netherlands. Eur J Cancer. 2008;44:683-91.

16. Dodwell D, Goyal A. Axillary conservation in early breast cancer. Br J Surg. 2015;102:1297-9.

17. Fisher B, Jeong JH, Anderson S, Bryant J, Fisher ER, Wolmark N. Twenty-five-year follow-up of a randomized trial comparing radical mastectomy, total mastectomy, and total mastectomy followed by irradiation. N Engl J Med. 2002;347:567-75.

18. van Laar C, van der Sangen MJ, Poortmans PM, Nieuwenhuijzen GA, Roukema JA, Roumen RM, et al. Local recurrence following breast-conserving treatment in women aged 40 years or younger: trends in risk and the impact on prognosis in a population-based cohort of 1143 patients. Eur J Cancer. 2013;49:3093-101. 
19. Schlembach PJ, Buchholz TA, Ross MI, Kirsner SM, Salas GJ, Strom EA, et al. Relationship of sentinel and axillary level I-II lymph nodes to tangential fields used in breast irradiation. Int J Radiat Oncol Biol Phys. 2001;51:671-8.

20. Kuhn T PP. Zoo11 - Can We Really Abolish Axillary Dissection? Breast Care 2011;6:154-7.

21. Galimberti V, Cole BF, Zurrida S, Viale G, Luini A, Veronesi P, et al. Axillary dissection versus no axillary dissection in patients with sentinel-node micrometastases (IBCSG 23-01): a phase 3 randomised controlled trial. Lancet Oncol. 2013;14:297-305.

22. Boughey JC. How do the AMAROS trial results change practice? Lancet Oncol. 2014;15:1280-1.

23. Gobardhan PD, Wijsman JH, van Dalen T, Klompenhouwer EG, van der Schelling GP, Los J, et al. ARM: axillary reverse mapping - the need for selection of patients. Eur J Surg Oncol. 2012;38:657-61.

24. Sakurai T, Endo M, Shimizu K, Yoshimizu N, Nakajima K, Nosaka K, et al. Axillary reverse mapping using fluorescence imaging is useful for identifying the risk group of postoperative lymphedema in breast cancer patients undergoing sentinel node biopsies. J Surg Oncol. 2014;109:612-5.

25. Noguchi M, Noguchi M, Nakano Y, Ohno Y, Kosaka T. Axillary reverse mapping using a fluorescence imaging system in breast cancer. J Surg Oncol. 2012;105:229-34.

26. Ikeda K, Ogawa Y, Kajino C, Deguchi S, Kurihara S, Tashima T, et al. The influence of axillary reverse mapping related factors on lymphedema in breast cancer patients. Eur J Surg Oncol. 2014;40:818-23.

27. Damstra RJ, Glazenburg EJ, Hop WC. Validation of the inverse water volumetry method: A new gold standard for arm volume measurements. Breast Cancer Res Treat. 2006;99:267-73.

28. Gross E, Hannoun-Levi JM, Rouanet P, Houvenaeghel G, Teissier E, Ellis S, et al. [Evaluation of immediate breast reconstruction and radiotherapy: factors associated with complications]. Cancer Radiother. 2010;14:704-10.

29. NOABON. Landelijke richtlijn mammacarcinoom 2.0. wwwoncolinenl/mammacarcinoom.

30. Tausch C, Baege A, Dietrich D, Vergin I, Heuer H, Heusler RH, et al. Can axillary reverse mapping avoid lymphedema in node positive breast cancer patients? Eur J Surg Oncol. 2013;39:880-6.

31. Noguchi M, Morioka E, Ohno $Y$, Noguchi M, Nakano $Y$, Kosaka T. The changing role of axillary lymph node dissection for breast cancer. Breast Cancer. 2013;20:41-6.

32. Noguchi M, Yokoi M, Nakano Y. Axillary reverse mapping with indocyanine fluorescence imaging in patients with breast cancer. J Surg Oncol. 2010;101:217-21.

33. Orr RK. The impact of prophylactic axillary node dissection on breast cancer survival--a Bayesian meta-analysis. Ann Surg Oncol. 1999;6:109-16.

34. Poortmans PM, Collette S, Kirkove C, Van Limbergen E, Budach V, Struikmans H, et al. Internal Mammary and Medial Supraclavicular Irradiation in Breast Cancer. N Engl J Med. 2015;373:317-27.

35. Whelan TJ, Olivotto IA, Parulekar WR, Ackerman I, Chua BH, Nabid A, et al. Regional Nodal Irradiation in Early-Stage Breast Cancer. N Engl J Med. 2015;373:307-16.

36. van den Hoven I, Voogd AC, Roumen RM. A Paradigm Shift in Axillary Breast Cancer Treatment; From "Treat All-Except," Toward "Treat None-Unless". Clin Breast Cancer. 2015;15:399-402.

37. Lopez Penha TR, van Roozendaal LM, Smidt ML, Boersma LJ, von Meyenfeldt MF, Voogd AC, et al. The changing role of axillary treatment in breast cancer: Who will remain at risk for developing arm morbidity in the future? Breast. 2015;24:543-7.

38. van der Heiden-van der Loo M, de Munck L, Sonke GS, van Dalen T, van Diest PJ, van den Bongard HJ, et al. Population based study on sentinel node biopsy before or after neoadjuvant chemotherapy in clinically node negative breast cancer patients: Identification rate and influence on axillary treatment. Eur J Cancer. 2015;51:915-21.

39. Alvarado R, Yi M, Le-Petross H, Gilcrease M, Mittendorf EA, Bedrosian I, et al. The role for sentinel lymph node dissection after neoadjuvant chemotherapy in patients who present with node-positive breast cancer. Ann Surg Oncol. 2012;19:3177-84.

40. Kuerer HM, Newman LA, Fornage BD, Dhingra K, Hunt KK, Buzdar AU, et al. Role of axillary lymph node dissection after tumor downstaging with induction chemotherapy for locally advanced breast cancer. Ann Surg Oncol. 1998;5:673-80.

41. Canavese G, Dozin B, Vecchio C, Tomei D, Villa G, Carli F, et al. Accuracy of sentinel lymph node biopsy after neo-adjuvant chemotherapy in patients with locally advanced breast cancer and clinically positive axillary nodes. Eur J Surg Oncol. 2011;37:688-94. 
42. Hunt KK, Yi M, Mittendorf EA, Guerrero C, Babiera GV, Bedrosian I, et al. Sentinel lymph node surgery after neoadjuvant chemotherapy is accurate and reduces the need for axillary dissection in breast cancer patients. Ann Surg. 2009;250:558-66.

43. Kelly AM, Dwamena B, Cronin P, Carlos RC. Breast cancer sentinel node identification and classification after neoadjuvant chemotherapy-systematic review and meta analysis. Acad Radiol. 2009;16:551-63.

44. van Deurzen $\mathrm{CH}$, Vriens BE, Tjan-Heijnen VC, van der Wall E, Albregts $M$, van Hilligersberg R, et al. Accuracy of sentinel node biopsy after neoadjuvant chemotherapy in breast cancer patients: a systematic review. Eur J Cancer. 2009;45:3124-30.

45. Xing Y, Foy M, Cox DD, Kuerer HM, Hunt KK, Cormier JN. Meta-analysis of sentinel lymph node biopsy after preoperative chemotherapy in patients with breast cancer. Br J Surg. 2006;93:539-46

46. Sakorafas GH, Peros G, Cataliotti L, Vlastos G. Lymphedema following axillary lymph node dissection for breast cancer. Surg Oncol. 2006;15:153-65.

47. Donker M, Straver ME, Wesseling J, Loo CE, Schot M, Drukker CA, et al. Marking axillary lymph nodes with radioactive iodine seeds for axillary staging after neoadjuvant systemic treatment in breast cancer patients: the MARI procedure. Ann Surg. 2015;261:378-82.

48. Boughey JC, Suman VJ, Mittendorf EA, Ahrendt GM, Wilke LG, Taback B, et al. Sentinel lymph node surgery after neoadjuvant chemotherapy in patients with node-positive breast cancer: the ACOSOG Z1071 (Alliance) clinical trial. JAMA. 2013;310:1455-61. 

Summary in Dutch / samenvatting in het Nederlands

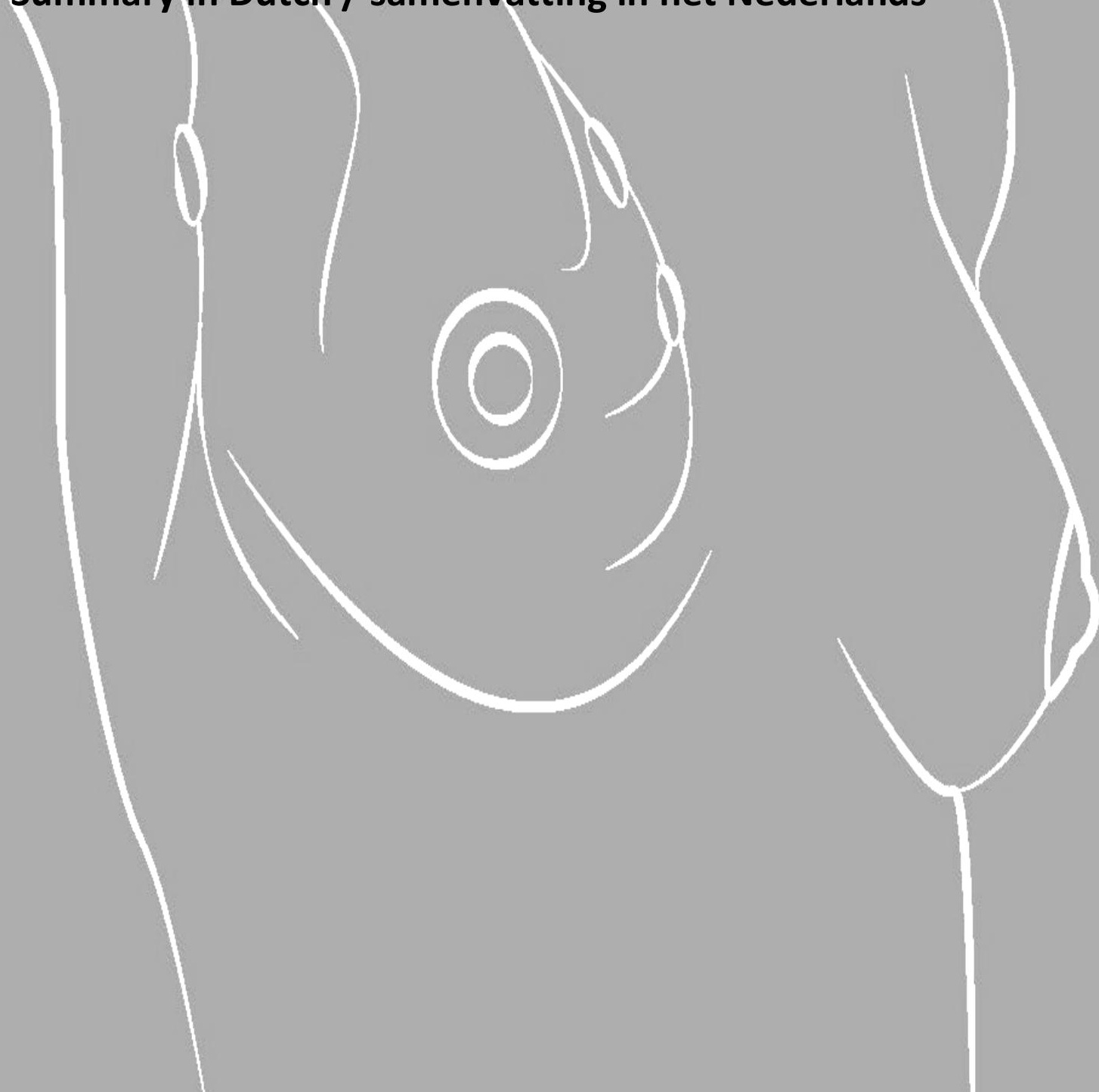





\section{Summary in Dutch / samenvatting in het Nederlands}

Door de eeuwen heen en in het bijzonder de afgelopen decennia is de behandeling voor patiënten met borstkanker sterk veranderd, met als positief resultaat dat de overleving van deze categorie patiënten blijft toenemen. Een okselklierdissectie (het verwijderen van alle lymfeklieren in de oksel) gold jarenlang als een van de hoekstenen van de behandeling van borstkanker en er zijn nog steeds veel patiënten die in aanmerking komen voor deze chirurgische behandeling. Een groot nadeel van een okselklierdissectie is dat deze gepaard kan gaan met aanzienlijke hinder voor de patiënt. Na een okselklierdissectie kunnen patiënten voornamelijk last hebben van het opzwellen van de arm (lymfoedeem). Lymfoedeem van de arm treedt na een okselklierdissectie op bij $7 \%$ tot $77 \%$ van de patiënten. De grote spreiding is mede te verklaren door de verschillende manieren waarop lymfoedeem wordt gemeten en de verschillende definities voor lymfoedeem die in diverse onderzoeken worden gebruikt.

Enkele jaren geleden is er een techniek beschreven waarbij het lymfatische drainagepatroon vanuit de arm tijdens de operatie in de oksel met behulp van kleuringstechnieken kan worden onderscheiden van het lymfedrainage netwerk wat voortkomt vanuit de borst. Deze techniek wordt axillary reverse mapping (ARM) genoemd. De achtergrond van deze techniek is de anatomische ligging van het lymfatische drainagesysteem; zowel "borst"- als "arm"-lymfeklieren zitten in de oksel en deze zijn normaal gesproken tijdens een operatie niet te onderscheiden van elkaar. Het concept van de ARM procedure is het in kaart brengen van de lymfedrainage van de arm, zodat deze gedurende een okselklierdissectie gespaard kan worden. Dit gebeurt door middel van het inspuiten van bijvoorbeeld een blauwe kleurstof in de binnenzijde van de bovenarm. Hierdoor worden alle "arm"-lymfeklieren en onderlinge lymfebanen blauw en kunnen ze tijdens de operatie goed worden onderscheiden van de "borst"-lymfeklieren en banen (deze zijn dan niet blauw). Indien de hypothese klopt dat lymfoedeem na een okselklierdissectie wordt veroorzaakt door het verwijderen van de "arm"-lymfeklieren en de bijbehorende lymfebanen dan zou de kans op het ontstaan van lymfoedeem, door het sparen hiervan, wellicht verminderd kunnen worden zonder dat de oncologische veiligheid in het geding is.

Hoofdstuk 2 is een overzicht van de chirurgische "okselbehandeling" bij patiënten met borstkanker in de afgelopen 20 jaar, in 10 verschillende ziekenhuizen, in het zuiden van Nederland. In deze studie worden duidelijke veranderingen beschreven in het behandelbeleid onder invloed van de publicatie van verschillende onderzoeken die de waarde van een okselklierdissectie ter discussie stellen. Het onderzoek had betrekking op de gegevens ruim 34.000 patiënten met borstkanker, die werden behandeld tussen januari 1993 en juli 2014. 
De eerste afname in het aantal okselklierdissecties is te zien in de jaren negentig, na het invoeren van de schildwachtklierprocedure. De schildwachtklierprocedure omvat het opzoeken en verwijderen van de lymfeklier die als eerste het lymfevocht uit de borst ontvangt en dus ook eventuele tumorcellen die zich hebben losgemaakt uit de tumor. Indien deze lymfeklier geen uitzaaiing bevat is gebleken dat de andere (volgende) lymfeklieren eveneens niet zijn aangetast. Op deze wijze hoeven de andere lymfeklieren dus niet te worden verwijderd met een okselklierdissectie. Deze okselsparende chirurgische techniek werd in de tweede helft van jaren negentig in Nederland geïntroduceerd, waarna een duidelijke vermindering van het aantal okselklierdissecties werd waargenomen. Waar in 1993-1994 nog bij 88\% van de borstkankerpatiënten een primaire okselklierdissectie werd verricht is dat in 20132014 gedaald naar $12 \%$.

De tweede verandering in het behandelbeleid ten aanzien van de oksel is te zien na de publicatie van de ACOSOG Z0011 studie in 2011. Deze Amerikaanse multicentrische studie randomiseerde borstkankerpatiënten met één tot drie positieve schildwachtklieren aansluitend tussen ofwel een aanvullende okselklierdissectie ofwel een afwachtend beleid (geen okselklierdissectie). De achterliggende gedachte hiervoor was dat tijdens een aanvullende okselklierdissectie - die tot dan toe standaard werd uitgevoerd nadat in de schildwachtklier uitzaaiingen waren aangetroffen met als doel eventuele uitzaaiingen in andere okselklieren te verwijderen - in veel gevallen geen uitzaaiingen meer werden aangetroffen en dus achterwege kon worden gelaten. Na een mediane follow-up duur van 6,3 jaar werd geen verschil gevonden in totale- en ziektevrije overleving. In de jaren 2009-2010, voorafgaand aan publicatie van de ACOSOG Z0011 studie, werd bij $5 \%$ van de borstkankerpatiënten met een positieve schildwachtklier geen aanvullende okselklierdissectie verricht. Na de publicatie, in de jaren 2013-2014, werd een aanvullende okselklierdissectie bij $37 \%$ van de borstkankerpatiënten achterwege gelaten.

Een versterking van de trend tot het achterwege laten van een okselklierdissectie die werd waargenomen tijdens ons onderzoek was na het publiceren van de zeer recente resultaten van de AMAROS studie. In deze studie werd aanvullend op een positieve schildwachtklier, een aanvullende okselklierdissectie vergeleken met het bestralen van de oksel. De resultaten lieten geen verschil in overleving zien, maar wel een verschil in morbiditeit ter gunste van de okselbestraling. Reeds voor dat de resultaten van de AMAROS studie officieel werden gepubliceerd was er al een trend te zien, maar zeker na publicatie van de resultaten nam het aantal borstkankerpatiënten dat een okselklierdissectie onderging sterk af in vergelijking met de jaren daarvoor. 
Hoofdstuk 3 is een weergave van een publicatie voor de Nederlandse medicus practicus over "axillary reverse mapping". In dit hoofdstuk wordt deze nieuwe techniek geïntroduceerd en nader uitgelegd. Tevens worden de indicaties voor het gebruik van deze techniek, de effectiviteit en de leercurve besproken.

In hoofdstuk 4 wordt een overzicht gegeven over de tot nu toe gepubliceerde literatuur over "axillary reverse mapping". Dit overzicht is ingedeeld in de volgende drie deelonderwerpen:

1) Technieken.

Er zijn verschillende technieken om de ARM procedure uit te voeren. In Nederland gebeurt dit door het inspuiten van een blauwe kleurstof maar in Japan gebeurt dit middels een fluorescerende stof die waar te nemen is met een speciale ('near infra-red') camera. Er zijn ook chirurgen die gebruik maken van een radioactieve stof die ze tijdens de operatie opsporen met een gamma-probe. Ook enkele studies zijn verricht waarbij bovengenoemde technieken worden gecombineerd.

2) Veiligheid.

Bij welke borstkankerpatiënten is het veilig om de "arm"-lymfeklieren te laten zitten, wetende dat er ook borstkankerpatiënten zijn bij wie er toch uitzaaiingen zijn gevonden in de "arm"-lymfeklieren?

3) Effectiviteit.

Er is literatuuronderzoek gedaan naar studies waarbij het effect van het selectief sparen van de "arm" lymfeklieren en de bijbehorende lymfebanen tijdens een okselklierdissectie op het ontstaan van postoperatieve lymfoedeem is beschreven.

Uit de bestudeerde literatuur kwam naar voren dat het zowel met de blauwe kleurstof als met een fluorescerende stof mogelijk is om de lymfeklieren van de "arm" en "borst" met hun bijbehorende lymfebanen goed te onderscheiden. Met de derde manier, het inspuiten van een radioactieve stof en deze daarna op te zoeken met een gamma-probe, bleek het wel mogelijk om de lymfeklieren te onderscheiden, maar konden lymfebanen niet worden opgespoord en tevens kon er geen onderscheid worden gemaakt tussen de verschillende lymfebanen. De conclusie op basis van de beschikbare literatuur is dat het wellicht beter is om de blauwe kleurstof of de fluorescerende stof te gebruiken voor de ARM procedure, omdat het belangrijk is om zowel de lymfeklieren als de bijbehorende lymfebanen goed te kunnen onderscheiden. In Nederland gebruiken we de blauwe kleurstof omdat we er al ruime ervaring mee hebben bij het uitvoeren van de schildwachtklierprocedure, het is een veilig gebleken kleurstof welke bovendien goedkoper is dan een fluorescerende stof (waar ook nog een speciale camera voor nodig is). 
In de huidige literatuur wordt de ARM procedure bij verschillende groepen borstkankerpatiënten beschreven. Om een uitspraak te doen over de oncologische veiligheid van de ARM procedure is er een onderscheid gemaakt tussen de volgende drie patiëntengroepen;

1. Patiënten bij wie de schildwachtklierprocedure en de ARM procedure tegelijkertijd werden uitgevoerd (SLNB patiënten).

2. Patiënten met de indicatie voor het uitvoeren van een okselklierdissectie na een verwijderde positieve schildwachtklier (SLN+ patiënten).

3. Patiënten met de indicatie voor het uitvoeren van een okselklierdissectie op basis van een positieve punctie uit een verdachte lymfeklier (CPN+ patiënten).

Uit de literatuur kwam naar voren dat er nog nooit uitzaaiingen in "arm"-lymfeklieren zijn gevonden bij SLNB patiënten die een schone schildwachtklier, dat wil zeggen een schildwachtklier zonder uitzaaiingen, hadden.

Bij SLN+ patiënten zijn incidenteel wel positieve "arm"-lymfeklieren beschreven. Dit waren dan wel altijd lymfeklieren die zowel als schildwachtklier fungeerde maar ook als de "arm" lymfeklier werden gezien. De conclusie op basis van de beschikbare literatuur was daarom dat het veilig is om de "arm"-lymfeklieren te sparen bij SLN+ patiënten, indien vast staat dat dit niet dezelfde lymfeklieren zijn als de schildwachtklier.

Voor de laatste groep, de CPN+ patiënten, kwam uit de literatuur naar voren dat tussen $0 \%$ en $60 \%$ van deze patiënten positieve "arm"-lymfeklieren heeft. Het is niet de bedoeling om bij patiënten lymfeklieren te sparen waarin uitzaaiingen zitten en daarom is er een verdere sub-analyse van deze groep patiënten nodig. Hier wordt later in hoofdstuk $\mathbf{8}$ en $\mathbf{9}$ dieper op in gegaan.

De laatste vraagstelling van het review in hoofdstuk 4 was of er verschil is in de incidentie van lymfoedeem tussen de patiënten met een standaard-okselklierdissectie en een ARM-okselklierdissectie, waarbij de "arm"-lymfeklieren zijn gespaard maar wel alle "borst"-lymfeklieren zijn verwijderd. Omdat er nog maar weinig onderzoek is gepubliceerd waarbij dit is onderzocht, en er geen resultaten van gerandomiseerde studies bekend waren, kon hierover geen uitspraak worden gedaan. Wel moet worden opgemerkt dat zeer recent, na publicatie van dit hoofdstuk, een Chinese studie is verschenen waarin naar voren kwam dat een okselklierdissectie met behulp van de ARM procedure minder lymfoedeem veroorzaakt.

In hoofdstuk $\mathbf{5}$ is het ontwerp beschreven van de ARM-studie, een gerandomiseerde multicenter studie naar de relevantie van de ARM procedure bij borstkankerpatiënten.

De ARM-studie omvat een randomisatiestudie en een registratiestudie. Borstkankerpatiënten met een indicatie voor een aanvullende okselklierdissectie (op basis van een positieve schildwachtklier) komen in aanmerking voor de randomisatiestudie. In de 
randomisatiestudie loten de patiënten tussen een standaard okselklierdissectie en een ARM-okselklierdissectie.

Patiënten met een directe indicatie voor een okselklierdissectie op basis van een klinisch positieve oksel komen in aanmerking voor de registratiestudie. Bij patiënten in de registratiestudie wordt altijd een standaard okselklierdissectie verricht, maar hierbij worden de "arm"-lymfeklieren wel zichtbaar gemaakt door middel van de ARM procedure en apart verwijderd en geanalyseerd op de aanwezigheid van eventuele uitzaaiingen.

De primaire uitkomstmaat van de randomisatiestudie is de aanwezigheid van borstkanker gerelateerd lymfoedeem. De secundaire uitkomstmaten zijn onder andere postoperatieve complicaties (pijn, tintelingen, gevoelloosheid en verlies van mobiliteit van de arm/schouder), kwaliteit van leven en terugkeer van borstkanker in de oksel. Naast een uitgangsmeting zullen er op zes, 12 en 24 maanden na de procedure metingen worden verricht. Zowel patiënten als diegenen die de metingen uitvoeren weten niet welke methode (al/of niet sparen van de ARM lymfeklieren) is uitgevoerd. Alle patiënten hebben namelijk de blauwe verkleuring op de bovenarm en hetzelfde litteken. Lymfoedeem zal gemeten worden door middel van de waterverplaatsingstechniek (met behulp van de Bravometer; voor een foto hiervan kijk bij hoofdstuk 6) en het meten van de omtrek van de arm (met behulp van een centimeter). De resterende metingen waaronder andere kwaliteit van leven, zal worden gedocumenteerd met behulp van een gestandaardiseerde vragenlijst speciaal ontwikkeld voor borstkankerpatiënten.

In hoofdstuk 6 hebben we de betrouwbaarheid en de reproduceerbaarheid bepaald van de Bravometer die in de ARM studie gebruikt wordt om lymfoedeem te meten. Tevens is tijdens dit onderzoek onderzocht of de watertemperatuur dan wel de omgevingstempratuur van invloed zijn op het volume van de arm. Het is belangrijk om een betrouwbare en reproduceerbare manier van meten te gebruiken omdat het gebruik van verschillende meetmethoden in de verschillende gepubliceerde onderzoeken één van de redenen is voor de grote variatie in de gerapporteerde lymfoedeem (7\% tot $77 \%$ ) na een okselklierdissectie.

In dit onderzoek hebben we door drie verschillende verpleegkundig specialisten, bij tien verschillende arts-assistenten (vijf mannen en vijf vrouwen), het volume van de arm laten meten met behulp van de Bravometer. Dit gebeurde elke dinsdag tussen de middag, drie weken achter elkaar. De resultaten van dit onderzoek toonden aan dat de Bravometer een zeer betrouwbaar meetinstrument is en dat het niet uitmaakt wie de meting uitvoert. Verder had in deze studie zowel temperatuur van het water als van de omgeving geen invloed op het volume van de arm. 
In hoofdstuk 7 beschrijven we onze visie over de plaats van de ARM procedure in een tijdperk van veranderende inzichten met betrekking tot de chirurgische behandeling van de oksel. De AMAROS studie heeft bestraling van de oksel vergeleken met een standaard okselklierdissectie en de resultaten van deze studie suggereren dat het bestralen van de oksel oncologisch even veilig is en dat het minder lymfoedeem veroorzaakt - weliswaar gemeten met slechts het meten van de armomtrek waarbij onderzoekers wisten welke behandeling de patiënt had ondergaan, in vergelijking met standaard okselklierdissectie. Echter, naast lymfoedeem kan bestraling ook leiden tot functionele schouderklachten en nadelige effecten hebben op andere aangrenzende weefsels en organen. Het selectief sparen van "arm"-lymfklieren en de bijbehorende lymfebanen met behulp van de ARM procedure tijdens een okselklierdissectie resulteert mogelijk in een veel lagere kans op lymfoedeem dan na een standaard okselklierdissectie en mogelijk ook tot minder morbiditeit dan radiotherapie.

In hoofdstuk 8 beschrijven we de resultaten van een retrospectief onderzoek bij borstkankerpatiënten met een indicatie voor een primaire okselklierdissectie. Dit zijn patiënten bij wie een okselklierdissectie noodzakelijk geacht werd vanwege een positieve punctie uit een verdachte lymfeklier (CPN+ patiënten, zoals eerder beschreven). In dit onderzoek is gekeken hoe vaak de "arm"-lymfeklieren uitzaaiingen bevatten bij deze groep patiënten, van wie een deel wel en een deel geen chemotherapie heeft ontvangen voorafgaand aan de borst- en okseloperatie.

De studiepopulatie bestond uit 98 borstkankerpatiënten (bij wie de ARM procedure is geslaagd), van wie er 79 (81\%) chemotherapie hadden gekregen voorafgaand aan de okselklierdissectie en de overige 19 (19\%) patiënten geen chemotherapie. In de laatstgenoemde groep zagen we dat er bij zeven van de 19 patiënten (37\%) uitzaaiingen werden gevonden in de "arm"-lymfeklieren maar dat van de 79 patiënten die van te voren behandeld chemotherapie hadden gehad er slechts 13 (17\%) uitzaaiingen in de "arm"-lymfeklieren hadden. De resultaten van dit onderzoek suggereren dat sommige $\mathrm{CPN}+$ patiënten na voorbehandeling met chemotherapie in aanmerking lijken te komen voor de ARM procedure met vervolgens sparen van de ARM klieren. Het is echter nog niet duidelijk welke patiënten dat precies zijn.

In hoofdstuk 9 beschrijven we een vervolgonderzoek op hoofdstuk 8 waarbij we retrospectief hebben gekeken of we met behulp van MRI-onderzoek van de borst, dat wordt uitgevoerd na de chemotherapie (dus voorafgaand aan de okselklierdissectie), uitspraken kunnen doen over de kans op uitzaaiingen in de "arm"-lymfeklieren. De studiepopulatie van dit onderzoek bestond uit $98 \mathrm{CPN}+$ patiënten die voorafgaand aan de okselklierdissectie chemotherapie hadden gekregen. De patiënten werden ingedeeld in een groep van patiënten waarbij op basis van MRI bevindingen geen verdenking meer bestond op uitzaaiingen in de oksel lymfeklieren na chemotherapie (RAD- groep) en een groep patiënten bij wie nog steeds verdenking bestond op 
uitzaaiingen in de oksel lymfeklieren na chemotherapie (RAD+ groep). Aanwezigheid van verdachte oksel-lymfeklieren op borst MRI na voorbehandeling met chemotherapie werd bepaald door twee ervaren radiologen met borstkanker als aandachtsgebied. Hun bevindingen werden gecorreleerd aan de histopathologische bevindingen. De resultaten van deze studie toonden aan dat er een significant verschil was in het aantal patiënten met uitzaaiingen in de "arm"-lymfeklieren tussen beide groepen. In de RAD- groep hadden slechts vijf van de 64 patiënten (7.8\%) uitzaaiingen in de "arm" lymfeklieren en in de RAD+ groep waren dit er tien van de 34 (29.4\%). Uit deze studie kunnen we concluderen dat de MRI die wordt gemaakt na de voorbehandeling met chemotherapie en dus voor de okselklierdissectie, eventueel gebruikt kan worden om nog nauwkeuriger CPN+ patiënten te identificeren die mogelijk een ARM-okselklierdissectie kunnen ondergaan in plaats van een standaard okselklierdissectie. 





\section{Valorisation}

Breast cancer is the most frequent malignancy diagnosed in women worldwide. In the Netherlands one out of every seven women will be diagnosed with breast cancer during her lifetime. Over the last decade survival of breast cancer patients has increased due to both the use of more effective systemic therapy as well as earlier detection by means of screening programs. Survival rates in the developed world are high, with between $80 \%$ and $90 \%$ of those in Europe and the United States alive for at least 5 years. Therefore, the number of patients potentially at risk for late effects (morbidity) of axillary treatment is increasing.

This thesis presents studies on a surgical procedure referred to as "axillary reverse mapping" (ARM) which enables discrimination of the lymphatic drainage of the upper extremity from the breast both situated in the axilla. The fundamental concept of the ARM procedure is based on the assumption that the upper extremity and breast each have their own discrete pathways of lymphatic drainage. If upper extremity lymphedema is caused by disruption of axillary lymphatics and/or removal of ARM lymph nodes, being able to identify and preserve them could possibly prevent upper extremity lymphedema during axillary lymph node dissection (ALND) or sentinel lymph node biopsy (SLNB).

\section{What is the social relevance of the research results?}

ALND has been a valuable technique for final staging of breast cancer patients. Information regarding the extent of axillary lymph node involvement remains important with regard to adjuvant treatment decisions. The surgical technique however is infamous for its morbidity, i.e. pain, numbness or paresthesia, arm and/or shoulder mobility restriction and especially upper extremity lymphedema. Upper extremity lymphedema may occur in $7-77 \%$ in axillary surgery and is thereby the most frequent complication. Patients with upper extremity lymphedema are more susceptible to upper extremity wound infections, pain, restriction of arm movement all of which result in structurally lower quality of life. Consequently total health care costs are increased in patients with lymphedema.

The basic question as to why development of upper extremity lymphedema occurs as well as surgical refinements of ALND in an effort to prevent this has not gained attention until the publications of Nos and Klimberg in 2007 introducing a more selective ALND introducing the addition of ARM. Ever since the first reports, various studies on the ARM procedure have been published regarding different visualisation techniques: blue dye, radio-isotope and fluorescence dye (or a combination of these techniques). Although currently not confirmed by multicentre randomised controlled trials, the ARM procedure has the potential to contribute to a significant reduction of upper extremity lymphedema in patients who underwent ALND. 


\section{For which category of patients are these results of interest?}

Breast cancer patients who are candidates for completion ALND (based on a positive sentinel lymph node (SLN)) may benefit from ARM procedure, as this will allow for better preservation the lymphatic draining system of the upper limb. A feasibility study performed in our hospital demonstrated that upper extremity lymph nodes, located in the axilla below the level of the axillary vein, can be visualised (using blue dye) in more than $90 \%$ of the patients. In these group of patients who underwent completion ALND, ARM lymph nodes were found to be free of tumour metastases.

In 2013 a multicentre randomized controlled trial (ARM trial) was initiated. The aim of the ARM trial was to determine the clinical utility of the ARM technique in identifying and sparing the upper limb-related axillary lymph nodes and its subsequent reduction of lymphedema of the upper limb. Following confirmation of an indication for a completion ALND, based on a positive SLN, patients has been randomly allocated to one of two groups (randomisation study): Level I-II ALND with preservation of the upper limb-related axillary lymph nodes (ARM-ALND) and a standard Level I-II ALND (standard-ALND). Patients who were candidates for primary ALND, based on axillary metastases proven by pre-operative fine needle aspiration cytology (FNAC, CPN+ patients: patients who had a preoperative diagnosis of lymph node metastasis proven by fine needle aspiration cytology and who are scheduled for primary ALND), were included in the registration study. The latter was set up to confirm the feasibility of the procedure in these group of patients and to allow further analysis on oncologic safety.

Results of the ARM registration study showed that a breast MRI post-neo adjuvant chemotherapy (NAC) may help to select a subgroup of $\mathrm{CPN}+$ patients in whom preservation of ARM lymph nodes during ALND may be considered, thereby reducing postoperative morbidity. Future research in $\mathrm{CPN}+$ patients is needed to provide evidence whether preservation of ARM lymph nodes during ALND is safe with respect to regional disease control.

\section{What does this mean for society?}

In 2014, approximately 500 breast cancer patients were treated with primary or completion ALND in the Netherlands. Some 40\% (200) of these women will develop upper extremity lymphedema. Treatment of lymphedema is mainly based on noninvasive strategies, such as physical therapy and use of compression garments. These conservative, non-invasive strategies may be helpful however they are timeconsuming, expensive, only supportive and effective in only a part of the patients.

If upper extremity lymphedema is caused by disruption of axillary lymphatics and/or removal of ARM lymph nodes, being able to identify and preserve them could possibly prevent upper extremity lymphedema during ALND. This change in treatment strategy, from a standard-ALND to ARM-ALND, has the potential to reduce the side- 
effects/morbidity induced by a standard-ALND, such as upper extremity lymphedema. Such a change will be of value to society since it is likely to result in less medical costs and less morbidity in the lives breast cancer patients.

\section{What is the future?}

ALND has lost its long standing monopoly in the treatment of SLN+ patients and CPN+ breast cancer patients. In current practice axillary radiotherapy (ART) or even omission of completion ALND in case of concordance with Z0011 criteria, are options which are increasingly adapted. However ALND will still play a role, albeit reduced, in selected patients. Consequently axillary treatment options have increased from a "one size fits all" to a more personalised axillary treatment.

Additional studies will further elucidate the role of different additional axillary treatments and their role with respect to further regional treatment de-escalation thereby reducing unnecessary morbidity with preservation of regional disease control. Furthermore the results of the ARM study will provide evidence whether preservation of ARM lymph nodes during ALND is safe with respect to regional disease control and as an effort to reduce morbidity. 



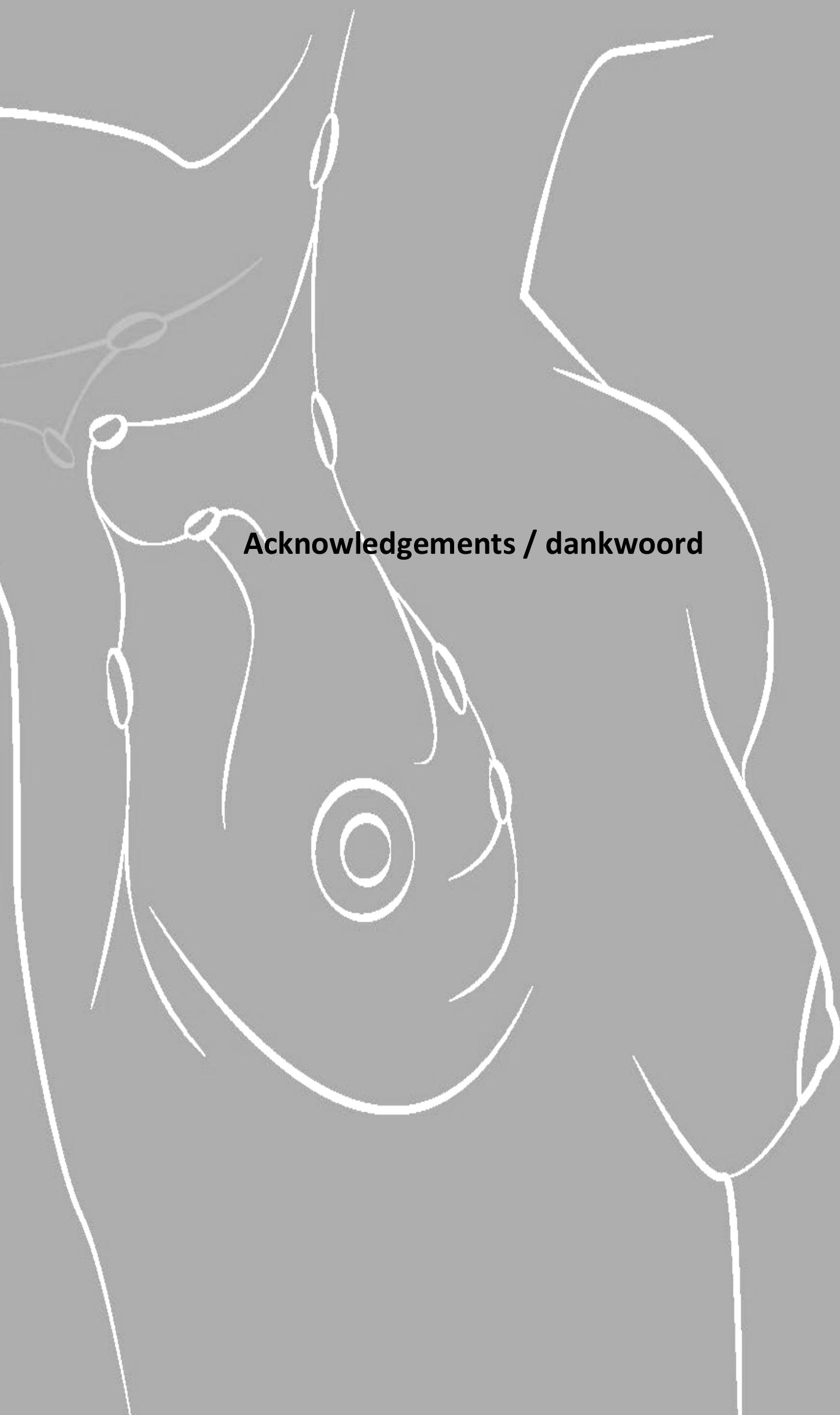





\section{Dankwoord}

Prof. dr. H.J.T. Rutten, promotor, beste $(\mathrm{H})$ arm, ik wil u heel erg bedanken voor de kans die u mij heeft gegeven om bij $u$ te mogen promoveren. Ik kan mij nog steeds uw eerste mail herinneren waarbij u afsloot met de woorden "Groet, $(\mathrm{H}) \mathrm{arm}$ "; het ijs was toen gelijk gebroken. Tijdens de vergaderingen had $u$ altijd nieuwe ideeën, goede adviezen en $u$ gaf mij ook de vrijheid en ruimte om mijn eigen onderzoeksplannen uit te werken. Ons congres in Wenen zie ik nog steeds als een van de hoogtepunten van mijn promotietraject.

Dr. E.J.T. Luiten, co-promotor, beste Ernest, wat hebben wij veel getennist tijdens de weekend- en avonduren. Ik ben u nog elke dag dankbaar dat u mij in 2013 de kans heeft gegeven om de kar te gaan trekken van de ARM studie. Het vertrouwen wat $u$ mij altijd heeft gegeven, is misschien wel het belangrijkste element van dit succesvol verlopen promotietraject. Als ik een artikel naar u stuurde voor revisie kreeg ik dit sneller terug dan dat ik mijn koffie op had. Een hoogtepunt van mijn promotietraject was zeker onze dag samen naar Twente, wat heb ik toen gelachen op de operatiekamer. De tips die u mij toen in de auto hebt gegeven, hebben mij zeker geholpen tijdens het solliciteren voor de opleiding. Verder ben ik echt trots op onze editorial samen met collega Isabel Rubio. Ik vind het nog steeds een eer dat ik mee mocht schrijven aan deze publicatie. Beste Ernest, ik hoop dat wij onze samenwerking zowel binnen het onderzoek, maar hopelijk ook op de operatiekamer zo door kunnen zetten, want ik geniet en leer er elke keer weer ontzettend veel van.

Dr. P.D. Gobardhan, co-promotor, beste Paul, ik herinner mij nog heel goed het telefoontje wat ik tijdens mijn co-schap psychiatrie van jou kreeg met de vraag of ik onderzoek zou willen doen in het Amphia. De vooruitgang die ik daarna zowel binnen als buiten het onderzoek heb doorgemaakt, heb ik mede aan jou te danken. Ik begon de eerste weken met brieven vouwen en rondjes rond een tafel lopen om het protocol in de juiste volgorde te leggen. En kijk waar ik nu sta; alle artikelen zijn gepubliceerd en ik ben in opleiding. Verder hebben we natuurlijk ook hele mooie dingen meegemaakt zoals tijdens de afgelopen skireis. Paul, je bent voor mij een voorbeeld, zowel als onderzoeker, maar zeker ook als chirurg en ik kijk er naar uit om de komende jaren nog veel meer van je te mogen leren. Er is nog een belofte waar ik je graag aan zou willen houden, we zouden samen een keer naar een congres gaan. Ik zou het heel mooi vinden als dit in de toekomst nog gaat gebeuren. 
Dr. A.C. Voogd, co-promotor, beste Adri, wat ben ik blij dat ik jou heb leren kennen. Ik wil je heel erg bedanken voor alle epidemiologische adviezen, het commentaar bij het schrijven van de hoofdstukken en natuurlijk voor jou begeleiding bij het gebruiken van SPSS. Ik heb je de afgelopen jaren leren kennen als een zeer toegankelijke en gedreven persoon die altijd bereikbaar voor mij was. Een heel goed voorbeeld van onze samenwerking zowel binnen als buiten het onderzoek is, denk ik de vakantiefoto die ik afgelopen zomer van je mocht ontvangen via de WhatsApp. Ik hoop dat onze samenwerking niet eindigt bij deze promotie.

Prof. dr. V.C.G. Tjan-Heijnen, prof. dr. C.H.C. Dejong, dr. M.B.I. Lobbes, prof. dr. Ph.M.P. Poortmans, prof. dr. E.J.Th. Rutgers, leden van de leescommissie, dank voor het beoordelen van mijn proefschrift.

Dr. E.G. Klompenhouwer, beste Lisa, als enige vrouwelijk lid van het ARM team heb je mij vanaf dag één ontzettend geholpen. Je was altijd, dag en avond, bereikbaar voor vragen over ons onderzoek, maar ook over mijn promotietraject. Ik wil je heel erg bedanken voor alle hulp en ik hoop dat we in de toekomst nog eens samen in de kliniek gaan werken.

Co-auteurs, publiceren doe je zeker niet alleen, dank voor jullie bijdragen aan de artikelen.

Patiënten, dank voor uw deelname aan de studies.

Dr. L.W.A. van Suijlekom-Smit, beste Lisette, zonder u was ik hier misschien nooit aan begonnen. Ik kan mij nog goed ons eerste diner bij u thuis herinneren waarbij we de literatuur over de ARM procedure samen doornamen. Ik wil u bedanken voor de hulp met het maken van de juiste keuze en ik hoop dat ik later ook iemand zo zal kunnen helpen.

Dr. L. van der Laan, beste Lijckle, we hebben elkaar leren kennen in 2012 tijdens mijn co-schap Heelkunde in het Amphia ziekenhuis. Tijdens mijn oudste co-schap een jaar later hebben we samen een plan gemaakt om in opleiding tot chirurg te komen. $U$ heeft mij altijd het vertrouwen gegeven dat dit goed zou komen, als ik mij maar zou houden aan onze afspraken en het is gelukt! Ik besef heel goed dat zonder uw support, toewijding met betrekking tot het onderzoek en de vrijheid die u mij tijdens mijn ANIOS periode hebt gegeven, ik nooit zo ver zou zijn gekomen. Ik wil u hiervoor heel erg bedanken en ik kijk er naar uit om de komende jaren samen dit succes door te trekken tijdens mijn opleiding. 
Dunja van der Meer, Judith Woolschot en Janneke Hover, beste dames, ik wil jullie heel erg bedanken voor alle inzet voor zowel de metingen als het verzamelen van de data met betrekking tot de ARM studie. Hoewel ik het jullie misschien niet altijd vertel, besef ik mij heel goed dat zonder jullie er geen ARM studie mogelijk is.

Chirurgen uit het Amphia ziekenhuis, wat heb ik de afgelopen jaren met veel plezier bij jullie gewerkt. Ik wil jullie bedanken voor de steun en de ruimte die ik heb gekregen om dit proefschrift te schrijven, maar vooral ook voor de mogelijkheid om onder jullie begeleiding de eerste stappen als beginnend arts te maken. Ik voel mij bevoorrecht dat ik de beginjaren van mijn opleiding in het Amphia mag volgen en ik kijk er heel erg naar uit om van jullie het vak te mogen leren.

A(N)IOS Heelkunde uit het Amphia ziekenhuis, wat hebben wij veel gelachen tijdens mijn eerste jaren als beginnende dokter. Ik wil jullie heel erg bedanken voor jullie collegialiteit en in het bijzonder wil ik de collega's bedanken die als proefpersoon hebben meegedaan aan de "Bravometerstudie".

J. Rasenberg, moederoverste, lieve Jacqueline, vanaf het moment dat ik als coassistent binnen kwam lopen heb je mij met alles geholpen. Je bent niet alleen de secretaresse van de opleider maar ook mijn persoonlijke secretaresse, kledingadviseur (dat mijn broeken hoger moeten) en vooral vriendin waar mee ik graag een kopje koffie drink en de dag doorneem. Ik ben blij dat we de komende jaren nog samen mogen werken.

Chirurgen en verpleegkundige specialisten van de deelnemende ziekenhuizen, heel erg bedankt voor jullie bijdragen aan dit proefschrift.

Secretariaat Heelkunde Amphia ziekenhuis, beste dames. Heel veel dank voor jullie gezelligheid, lekkere koffie en natuurlijk alle hulp die jullie mij altijd geven.

Medewerkers van de operatiekamer, verpleegkundige op de chirurgische afdeling, Amphia academie, Kenniskern Inkoop (bedankt voor alle kopieën en faxen), poli assistenten en opnameplanning chirurgie (bedankt voor alle mailtjes), bedankt voor jullie bijdragen aan dit proefschrift.

J. van Trier, beste Jan, er zijn tijden geweest dat ik "jouw" werkplek vaker zag dan mijn eigen woonkamer. Ik ben erg blij dat ik altijd welkom was in de bibliotheek. Ik hoop dat de rust nu weer een beetje wedergekeerd is. PS ik kom mijn spullen echt nog een keer weghalen uit de kast. 
Dr. J van der Slegt, drs. J.W. Raats, drs. S.L. Steunenberg, beste Jasper, Jelle en Stijn, wat hebben wij een mooie tijd gehad in de bibliotheek. Jasper, samen met Tim heb ik de langste tijd met jou in de bibliotheek gezeten en ik krijg nog steeds een glimlach op mijn gezicht als ik terug denk aan die tijd. Ik heb ontzettend veel zin om de komende jaren als chirurg in opleiding samen te gaan werken. Jelle, jouw uitspraken zal ik nooit vergeten. Ik weet dat je af en toe gek van mij werd, maar zonder een klein beetje afleiding kan je geen onderzoek doen. Ik hoop dat wij in de toekomst weer samen gaan werken. Stijn, jij was echt gelijk een aanwinst in de bieb, al was het alleen maar omdat je altijd een kannetje water haalde. Ik kijk er naar uit om samen op de SEH te gaan klussen en ik hoop ook dat jij de komende jaren een keer mee gaat solliciteren voor de opleiding.

Drs. T.S. Jansent, beste Tim, de momenten die we samen hebben beleefd zal ik nooit vergeten; het squashen samen met Slegt, filmpjes kijken na onze dienst en natuurlijk dumpert kijken tijdens het onderzoek. Ik ben echt trots dat onze "droom" om samen iets te publiceren is uitgekomen. Ik heb me aan mijn afspraak gehouden door in opleiding te komen. Tim, ik had heel graag nog veel meer avonturen met je willen beleven.

Marithé, heel erg bedankt voor de steun de afgelopen jaren.

Mannen van de TGG, ik weet dat de vrouwelijke oksel niet het meest mooie onderwerp is om over te praten en daarom verbaast het mij elke keer weer dat jullie zo goed op de hoogte zijn van mijn onderzoek. Ik heb altijd maximaal genoten van de ontspanning tijdens onze avonturen en ik kijk er naar uit om de komende jaren nog veel meer mooie trips samen te gaan maken.

Mannen van de Schieweg, beste Hidde, Marnix, Thomas, Remy, Hugo en Bas, ik wil jullie bedanken voor de afleiding die jullie mij hebben gegeven tijdens mijn beginjaren als dokter in combinatie met het doen van onderzoek in mijn "vrije tijd". Hidde, onze studententijd zal ik nooit vergeten. Marnix en Thomas, mijn tweede studententijd met jullie was iets wat ik nooit had willen missen. Remy, Hugo en Bas, ik wil jullie bedanken voor al het lekkers wat jullie s'nachts meenemen en voor dat jullie mij dan niet vergeten wakker te maken.

Beste waterpolovrienden, het schrijven van mijn proefschrift heb ik, zoals jullie weten, grotendeels in mijn "vrije" tijd gedaan. Ik ben erg blij dat jullie nooit opmerkingen hebben gemaakt over dat ik ergens niet bij was. Nu dit proefschrift afgerond is, zal ik weer vaker met jullie aan de bar komen hangen. 
Bestuursleden en oud-bestuursleden van het 't Zwarte Plasje, dank voor de wijze lessen die ik van jullie heb mogen leren tijdens, maar ook zeker na onze vergaderingen, dit heeft mij zeker geholpen tijdens mijn promotietraject.

Mijn paranimfen, Dirk-Pieter Suijlekom en Victor van den Berg. Dakhaas, ik ken je nu al bijna 20 jaar en de avonturen die wij hebben meegemaakt zijn ongekend. Toch wil ik je niet alleen bedanken voor alle avonturen maar zeker ook voor alle wijze lessen die ik van jou heb mogen ontvangen, onze vriendschap is mij heel veel waard. Ik wil ben erg blij dat je naast mij staat tijdens mijn verdediging. Vic, wat hebben wij veel gelachen zowel tijdens de studie maar ook daarbuiten. Ik wil je bedanken omdat je mij altijd heb gesteund en ik ook altijd op je kon rekenen, ook als het even tegenzat. Ik ben erg trots dat jij mijn paranimf wil zijn.

Lieve opa en oma, jullie zijn het voorbeeld van hoe ik oud zou willen worden. Dank voor alle lieve telefoontjes en WhatsAppjes die ik van jullie heb ontvangen. Ik ben echt heel trots dat ik mijn proefschrift aan jullie mag komen overhandigen.

Lieve Lonneke, Wouter en Joris, lieve zus en broertjes, als eerste wil ik mijn excuses maken dat het de laatste jaren altijd even over mijn onderzoek moest gaan tijdens het kerstdiner en verjaardagen. Ik denk dat er maar weinig niet medici zijn die zoveel kennis over de vrouwelijke oksel hebben vergaard in de afgelopen jaren. Lon, als ik je nodig heb ben je er altijd en alleen daarom wil ik je al bedanken. Woeter, misschien lijken wij van ons vieren wel het meeste op elkaar maar toch zijn er ook verschillen. Ik had eerlijk gezegd nooit gedacht dat je zoveel van een jonger broertje kon leren. Ik hoop dat we elkaar weer een stuk vaker gaan zien nu je in Amsterdam woont. Barry, je bent niet alleen mijn broer en mijn oude huisgenoot maar bovenal een hele goede vriend. Wat hebben wij een top studententijd gehad. Ik wil je heel erg bedanken omdat je mij altijd steunt, welke keuze ik ook maak.

Lieve mama en papa, zonder jullie steun en liefde had ik dit nooit kunnen doen. Jullie staan altijd voor mij klaar en daar wil ik jullie echt voor bedanken.

Lieve Sophie, wat ben ik blij dat ik jou heb ontmoet. Ze zeggen altijd dat de laatste loodjes het zwaarst wegen en ik ben heel blij dat je mij daarbij hebt geholpen. Ik kijk uit naar onze toekomst samen. 





\section{List of publications}

MA Beek, ELL Twiss, PAM Kint. Toevalsbevinding bij een man met een pijnlijke knie. Ned Tijdschr Geneeskd. 2013;157: A5131p

PD Gobardhan, EG Klompenhouwer, MA Beek, AC Voogd, EJT Luiten. 'Axillary reverse mapping' Is visualiseren van lymfebanen bij okselklierdissectie klinisch relevant? Ned Tijdschr Geneeskd, 2013;157: A5646.

EG Klompenhouwer, PD Gobardhan, MA Beek, AC Voogd, EJT Luiten. The clinical relevance of axillary reverse mapping (ARM): study protocol for a randomized controlled trial. Trials. 2013. 14:111.

JD Luiten, MA Beek, AC Voogd, PD Gobardhan, EJT Luiten. "lodine seed-versus wireguided localization in breast-conserving surgery for non-palpable ductal carcinoma in situ”. Br J Surg. 2015 Dec;102(13):1665-9.

MA Beek, TS Jansen, JW Raats, ELL Twiss, PD Gobardhan, EJH van Rhede van der Kloot. "The use of peritoneal drains in patients with perforated appendicitis". SpringerPlus. 2015;4:371

MA Beek, NC Verheuvel, EJT Luiten, EG Klompenhouwer, HJT Rutten, RMH Roumen, PD Gobardhan, AC Voogd. "Two decades of axillary management in breast cancer". Br J Surg. 2015 Dec;102(13):1658-64.

MA Beek, A te Slaa, L van der Laan, PGH Mulder, HJT Rutten, AC Voogd, EJT Luiten, PD Gobardhan. "Reliability of the Inverse Water Volumetry method to measure the volume of the upper limb". Lymphat Res Biol. 2015 Jun;13(2):126-30.

MA Beek, PD Gobardhan, EG Klompenhouwer, HJT Rutten, AC Voogd, EJT Luiten. "Axillary reverse mapping (ARM) in clinically node positive breast cancer patients". Eur J Surg Oncol. 2015 Jan;41(1):59-63.

CJLM Catsman, MA Beek, AM Rijken. "Talc seromadesis in patients with chronic seroma formation after breast surgery". SpringerPlus. 2016;5:3.

MA Beek, E Tetteroo, EJT Luiten, PD Gobardhan, HJT Rutten, JB Heijns, AC Voogd, EG Klompenhouwer. "Clinical impact of breast MRI with regard to axillary reverse mapping in clinically node positive breast cancer patients following neo-adjuvant chemotherapy". Eur J Surg Oncol. 2016 May;42(5):672-8. 
EJT Luiten, MA Beek, IT Rubio. "Clinical utility of Axillary Reverse Mapping (ARM) in an era of changing perceptions concerning axillary surgery". Eur J Surg Oncol. 2016 May;42(5):585-7.

MA Beek, PD Gobardhan, EJP Schoenmaeckers, EG Klompenhouwer, HJT Rutten, AC Voogd, EJT Luiten. "Axillary reverse mapping (ARM) in axillary surgery for breast cancer - an update of current status". Breast Cancer Res Treat. 2016 Aug;158(3):42132. 




\section{Curriculum Vitae Auctoris}

The author of this thesis, Martinus Antonius Beek, was born on January 27th in 1988 in Rotterdam, the Netherlands. He graduated high school at the Rotterdams Montessori Lyceum in Rotterdam in 2007. The same year he was admitted at the medical school at the Erasmus University Rotterdam. During the first years of his internship he started the beginning of this thesis under supervision of dr. E.J.T. Luiten, dr. P.D. Gobardhan and dr. A.C. Voogd. In December 2013 he graduated his medical training and in January 2014 he started working as a surgical resident (ANIOS) at the Department of Surgery at the Amphia hospital in Breda (dr. L. van der Laan).

The main aim for his $\mathrm{PhD}$ project was to describe the introduction of axillary reverse mapping (ARM); a procedure which enables discrimination of the upper extremity and breast lymphatic drainage in the axilla. He is study coordinator of the ARM study (a randomized controlled multicentre trial). For this study he was awarded with grants from Pink Ribbon and the Maurits and Anna de Kock foundation. Furthermore he was winner of the "Pieter Stijnen science award" in the Amphia Hospital in 2015.

Currently he is working as a surgical resident in training (AIOS) at the Amphia Hospital in Breda, The Netherlands (dr. L van der Laan and dr. Wijhoven). 
